\title{
Singularités et transfert
}

\section{Für Günter Harder in vierzigjähriger Freundschaft}

\author{
Robert P. Langlands
}

0. Introduction. Dans les quelques pages qui suivent, je continue, mais seulement pour le groupe $S L(2)$, le développement commencé dans l'article Formule des traces et fonctorialité: les débuts d'un programme écrit avec Edward Frenkel et Ngô Bau Châo. Nous utilisons les notations de cet article, sauf que la lettre $s$, qui avait malheureusement dans [FLN] deux fonctions, sera pour une de ces fonctions remplacée par $t$ et par la suite disparaîtra pour toujours. Pour la variable des fonctions $L$, par exemple $L(s, \pi, \rho)$, nous continuons à employer la lettre $s$, mais pour la fonction $\theta(b, \cdot)$ la deuxième variable sera remplacée par $t$. En fait, après quelques tergiversations je laisserai tomber ce paramètre dans $\theta$ et poserai franchement $t=1$. Dans cet article il s'agit d'abord de la définition et de la construction du transfert stable dans le cadre du groupe $S L(2)$, et ensuite de l'étude des singularités de la fonction $\theta_{v}(b, t)$ de $[\mathrm{FLN}]$ et d'une fonction $\varphi_{v}$ définie dans cet article à partir du transfert. Cela nous permet de montrer, au moins pour $S L(2)$, qu'il est possible d'utiliser la formule de Poisson dans l'étude de la formule des traces. Le lecteur comprendra qu'un des objectifs par la suite sera de faire en général ce que l'on fait ici pour le groupe $S L(2)$, donc de le faire pour les groupes que l'on a appelés en suivant Kottwitz des $z$-extensions.

Malheureusement, sauf pour le lemme fondamental lui-même, les principes de la formule des traces stable, tels que formulés provisoirement dans [L1], et développés dans [Ko] et $[\mathrm{A}]$, sont peu compris. Les lecteurs qui veulent en savoir plus sont renvoyés à ces articles. Les arguments qui suivent supposent quelque aptitude à manier les notions de base de l'analyse harmonique invariante et stable. Pour le groupe $S L(2)$ il y a bon nombre de formules explicites dont nous aurons besoin et pour lesquelles le lecteur est renvoyé à [LL].

Les démonstrations sont à maints égards insatisfaisantes. Pour les singularités des fonctions $\theta$ elles reposent sur des théorèmes sur le comportement asymptotique des intégrales orbitales dûs à Harish-Chandra [V] et à Shalika [Sh] et sur des calculs explicites pour le groupe $S L(2)$. Il est très probable qu'avec les méthodes de la géométrie algébrique contemporaine on pourrait remplacer ces calculs par des principes qui donnent les résultats généraux nécessaires. Pour les groupes autres que $S L(2)$, ou plutôt pour des groupes de rang réductif plus grand que 1, ces méthodes générales seront sans doute impératives.

La démonstration de l'existence du transfert pour $S L(2)$ utilise les formules pour les caractères données par Sally-Shalika [SS]. Par conséquent elle n'est encore valable ni pour les corps de fonctions, ni pour un corps p-adique à caractéristique résiduelle 2 , deux cas qui seront en principe exclus dès maintentant, le deuxième cas rigoureusement parce que les formules nécessaires ne sont pas encore connues. Pour le premier cas, les mêmes formules sont sans doute valables mais pas encore démontrées. En plus, les démonstrations de Sally- 
Shalika n'ont jamais été publiées. Leurs résultats concrets sont peut-être des conséquences d'un théorème de Tunnell ([T]). J'avoue franchement que l'objectif, dans cet article comme dans [FLN], est de tracer un chemin qui pourrait mener à la fonctorialité, non pas de la réaliser immédiatement. Le lecteur et la lectrice doivent par conséquent accepter, encore une fois comme pour l'article [FLN], qu'il s'agit d'un article lacunaire. Cependant nous entamons l'emploi de la formule de Poisson de sorte que l'article est censé être un tremplin qui nous permettra de nous lancer vers les problèmes analytiques et de les aborder. Il est divisé en quatre parties principales: le transfert; les singularités; le comportement asymptotique; la formule de Poisson.

\section{Le transfert}

1.1. Le transfert stable. Avant de vérifier que ce transfert existe pour le groupe $S L(2)$, je rappelle comment nous nous attendons le définir en général, en nous servant des concepts de l'analyse harmonique stablement invariant qui ne sont pas véritablement acquis sauf pour le groupe $S L(2)$. Il serait peut-être plus honnête de dire que pour le moment, n'ayant eu besoin d'eux que pour ce groupe, je n'ai pas fait d'effort de suivre en détail tout le progrès réalisé depuis le bouquin [L1]. Je renvoie le lecteur au livre de Arthur ([A]).

Le transfert est d'abord défini pour un corps local. Pour les groupes locaux $G\left(F_{v}\right)$ il est possible de définir la notion d'une représentation irréductible $\pi$, d'une représentation tempérée, et, en principe, des $L$-paquets. Rattachée à une représentation irréductible (admissible) est son caractère qui est une distribution invariante sur $G\left(F_{v}\right)$ donnée par une fonction $\chi_{\pi}$, une fonction qui est lisse sur l'ensemble des éléments réguliers $G^{\text {reg }}\left(F_{v}\right)$ de $G\left(F_{v}\right)$. La distribution est le produit de cette fonction et de la mesure de Haar. C'est la fonction et non pas la distribution qui est canonique. Rattaché à un $L$-paquet que nous notons $\pi^{\text {st }}$ est son caractère $\chi_{\pi^{\text {st }}}$. On s'attend que $\chi_{\pi^{\text {st }}}$ soit une combinaison linéaire

$$
\chi_{\pi^{\mathrm{st}}}=\sum_{\pi \in \pi^{\mathrm{st}}} a_{\pi} \chi_{\pi}
$$

mais en fait pour un corps non archimédien les caractères stables, ou même les caractères irréductibles, sont mal compris. En plus pour tous les corps locaux les paquets non tempérés ont été très peu étudiés. Cela n'empêche pas que pour l'étude de l'analyse harmonique locale les caractères stables, tempérés ou non, sont indispensables.

Selon la théorie de l'analyse harmonique invariante pour les groupes réductifs prévue dans [L1], et avec une notation qui s'écarte de celle de la formule (2.5) de [FLN], à une application de groupes $L, \phi:{ }^{L} H \rightarrow{ }^{L} G$, est rattachée une application de $L$-paquets, $\pi_{H}^{\text {st }} \rightarrow$ $\pi_{G}^{\text {st }}$. S'il devient nécessaire de travailler avec les paramètres de Arthur, nous remplaçons (2.5) $\operatorname{par} \phi^{\text {Art }}=\sigma^{\text {Art }} \times \psi^{\text {Art }}$.

Il est plus prudent de ne considérer au début que des paquets tempérés et pour le groupe $S L(2)$ cela suffit pour le moment. Il n'est pas du tout évident comment construire en général des distributions stablement invariantes à partir des paquets non tempérés. Cependant, on peut supposer avec quelque confiance que pour chaque paquet tempéré $\pi^{\text {st }}$, 
il est possible d'introduire une somme de la forme (1.1.1) qui est stablement invariante. On s'attend qu'à une constante près il n'y ait pour un $\pi^{\text {st }}$ donné qu'une seule telle somme. L'étude de ces caractères stables est peu avancée mais je m'attends qu'il y ait une normalisation naturelle. Pour le groupe $G=S L(2)$, le seul groupe qui interviendra dans cet article, les coefficients sont tous égaux à 1 , au moins si la caractéristique résiduelle n'est pas 2, ce que je suppose. Pour les groupes $H$ dont il s'agit dans cet article, qui seront tous des tores, un $L$-paquet ne contient qu'un seul élément et les coefficients sont encore tous 1.

Selon la fonctorialité, dont seulement une mince partie a été établie, mais que nous cherchons à établir en général — plus précisément nous cherchons à créer une théorie assez solide et assez large pour permettre de l'établir - il y a un transfert de $L$-paquets stables tempérés

$$
\hat{\phi}: \pi_{H}^{\mathrm{st}} \rightarrow \pi_{G}^{\mathrm{st}}
$$

Les caractères stables de ces paquets sont supposés être des fonctions stablement invariantes sur $H\left(F_{v}\right)$ et sur $G\left(F_{v}\right)$, donc des fonctions sur les bases de Steinberg-Hitchin $\mathfrak{A}_{H}$ et $\mathfrak{A}_{G}$ qui ont été introduites dans [FLN]. Nous cherchons un transfert tel que les valeurs de $\chi_{G}^{\text {st }}=\chi_{G_{v}}^{\text {st }}$ soient une fonction linéaire des valeurs de $\chi_{H}^{\text {st }}=\chi_{H_{v}}^{\text {st }}$. Puisque les caractères stables sont censés former une base des fonctions, même des distributions, stablement invariantes, cela donne en principe des formules,

$$
\chi_{\pi_{G}^{\mathrm{st}}}\left(a_{G}\right)=\int_{\mathfrak{A}_{H}} \chi_{\pi_{H}^{\mathrm{st}}}\left(a_{H}\right) \Theta\left(a_{H}, a_{G}\right) d a_{H}
$$

car pour un $a_{G}$ donné l'expression à gauche rattache un nombre complexe à chaque élément $\chi_{\pi_{H}^{\text {st }}}$ de cette base des fonctions invariantes sur $G\left(F_{v}\right)$. Ce sont les fonctions $\Theta$ que nous cherchons à trouver et à partir desquelles nous définirons le transfert, au moins pour le groupe $S L(2)$. Il y a une difficulté de notation. Nous sommes habitués à traiter les caractères stables comme fonctions sur les classes de conjugaison stable, mais dans l'article [FLN] nous sommes passés de ces classes aux points $a_{H}$ ou $a_{G}$ et à la fonction $\theta_{v}$ rattachée à $f$. Les deux points de vue - caractères stables comme fonctions ou distributions, la mesure de Haar étant donnée, sur $G\left(F_{v}\right)$ ou comme fonctions sur la base de Steinberg-Hitchin — sont mêlés dans (1.1.3). Il faudra alors revenir à la formule (1.1.3) et la réécrire en employant systématiquement la base de Steinberg-Hitchin. Il est certainement admissible, au moins pour $\Delta\left(a_{G}\right) \neq 0$, de traiter le caractère stable comme fonction de $a_{G}$, comme nous avons fait dans la formule (1.1.3).

Puisqu'il est inutile de traîner l'indice $v$ partout, nous l'enlevons souvent lorsqu'il est évident qu'il s'agit d'un corps local. En revenant au cas global, où il y a question de représentations du groupe adélique $G\left(\mathbb{A}_{F}\right)$ et des groupes locaux $G\left(F_{v}\right)$, nous remettrons l'indice. Quoiqu'il ne s'agisse que de caractères stables, nous n'omettrons que rarement l'indice supérieur.

Dans [FLN] les mesures locales et globales sur les groupes réductifs ont été définies d'une façon systématique que je continue d'utiliser ici. Pour les calculs locaux, les seuls 
choix pertinents sont ceux d'un caractère local et d'une mesure sur le corps $F_{v}$ invariante et auto-duale par rapport au caractère choisi et d'une forme différentielle invariante sur le groupe $G$, sur les groupes $H$, et sur les sous-groupes de Cartan de $G$ et de $H$. Lorsqu'il s'agit d'un corps global, les caractères locaux sont ceux déduits d'un caractère global par rapport auquel le corps global $F$ est auto-dual. La seule condition supplémentaire que nous imposons, c'est que les formes invariantes sur les sous-groupes de Cartan seront toutes conjuguées. Elles ne sont pas nécessairement définies sur $F$. Si $T$ et $T^{\prime}$ sont deux sous-groupes de Cartan sur $F$, il y a toujours un $w \in G\left(\bar{F}^{\text {sep }}\right)$ tel que $T^{\prime}=w T w^{-1}$. Nous exigeons que $\omega_{T}=\omega_{T^{\prime}}=\omega_{T}$ o ad $w$. Il est bien possible que ni $\omega$ ni $\omega^{\prime}=\omega_{T^{\prime}}$ ne soient définis sur $F$. Cela n'est pas grave, car, par exemple, si $F=F_{v}$ est local, alors $\omega=\alpha \omega_{1}$, où $\omega_{1}$ est défini sur $F_{v}$ et $d \omega=|\alpha| d \omega_{1}$. Globalement $d \omega=d \omega_{1}$. Cette cohérence est nécessaire si nous voulons utiliser la mesure $d a_{G}$ sur la base de Steinberg-Hitchin.

On cherche aussi à introduire un transfert dual de fonctions lisses à support compact $f_{v}=f^{G} \rightarrow f_{v}=f^{H}$ tel que

$$
\int_{H\left(F_{v}\right)} f^{H}\left(h_{v}\right) \chi_{\pi_{H}^{\mathrm{st}}}\left(h_{v}\right) d h_{v}=\int_{G\left(F_{v}\right)} f^{G}\left(g_{v}\right) \chi_{\pi_{G}^{\mathrm{st}}}\left(g_{v}\right) d g_{v},
$$

ou plus brièvement

$$
\chi_{\pi_{H}^{\mathrm{st}}}\left(f^{H}\right)=\chi_{\pi_{G}^{\mathrm{st}}}\left(f^{G}\right) .
$$

Lequel des deux transferts est primaire, celui des caractères ou celui des fonctions, n'est pas dit. En plus on n'exige pas, sauf si $H$ est un tore, que $f^{H}$ soit unique. On s'attend que ses intégrales orbitales stables soient bien définies et qu'elles soient des fonctions linéaires des intégrales orbitales de $f=f^{G}$. Donc malgré la flèche il s'agit d'une correspondence. De telles correspondences sont semblables à celles de la théorie de l'endoscopie, mais différentes. Les classes de conjugaison dans les deux groupes sont données par des points des bases de Steinberg-Hitchin $\mathfrak{A}_{H}$ et $\mathfrak{A}_{G}$. D'après l'équation (3.31) de [FLN] l'équation (1.1.4) est l'égalité des deux intégrales,

$$
\int\left|\Delta\left(a_{H}\right)\right| \lambda\left(a_{H}\right) \operatorname{Orb}^{\mathrm{st}}\left(a_{H}, f^{H}\right) \chi_{\pi_{H}^{\mathrm{st}}}\left(a_{H}\right) d a_{H}
$$

et

$$
\int\left|\Delta\left(a_{G}\right)\right| \lambda\left(a_{G}\right) \operatorname{Orb}^{\mathrm{st}}\left(a_{G}, f^{G}\right) \chi_{\pi_{G}^{\mathrm{st}}}\left(a_{G}\right) d a_{G},
$$

où nous utilisons comme première variable dans les intégrales orbitales et dans les caractères non pas une classe de conjugaison régulière mais son image dans la base de SteinbergHitchin. Nous avons mis pour $a_{H}$ régulier

$$
\lambda_{v}\left(a_{H}\right)=\lambda\left(a_{H}\right)=L\left(1, \sigma_{T_{H} / H}\right)=L_{v}\left(1, \sigma_{T_{H} / H}\right),
$$

où $T_{H}$ est le tore rattaché à un $t_{H}$ régulier et semi-simple dont l'image est $a_{H}$. Le facteur $\lambda\left(a_{G}\right)$ est défini de la même façon. Il sera souvent prudent de mettre par la suite l'indice inférieur $v$ pour distinguer les fonctions $L$ locales des fonctions $L$ globales. 
Puisque l'ensemble des caractères stables tempérés pour $H\left(F_{v}\right)$ est censé être complet, les équations (1.1.3) donnent alors la formule

$$
\left|\Delta\left(a_{H}\right)\right| \lambda\left(a_{H}\right) \operatorname{Orb}^{\mathrm{st}}\left(a_{H}, f^{H}\right)=\int\left|\Delta\left(a_{G}\right)\right| \lambda\left(a_{G}\right) \operatorname{Orb}^{\mathrm{st}}\left(a_{G}, f^{G}\right) \Theta\left(a_{H}, a_{G}\right) d a_{G},
$$

au moins pour $a_{H}$ régulier et semi-simple dans $H\left(F_{v}\right)$ et $a_{G}$ régulier et semi-simple dans $G\left(F_{v}\right)$. Les fonctions $\Delta$ des deux côtés sont différentes. On doit y mettre $\Delta_{H}\left(a_{H}\right)$ et $\Delta_{G}\left(a_{G}\right)$. Pour un lecteur averti un seul indice $H$ et un seul indice $G$ doit suffire mais dans les cas où même pour un lecteur tout à fait conscient des deux possibilités il serait trop facile de les confondre, nous mettons un indice double. Les mesures $d a_{H}$ et $d a_{G}$ sur $\mathfrak{A}_{H}$ et $\mathfrak{A}_{G}$ sont définies selon les principes de $[\mathrm{FLN}]$. Nous nous sommes départis de la notation de cet article où la première variable dans l'intégrale orbitale était un élément de la classe $\left\{t_{G}\right\}$ ou $\left\{t_{H}\right\}$ rattachée à $a_{G}$ ou $a_{H}$. Elle est maintenant la classe elle-même. La fonction $\Theta\left(a_{H}, a_{G}\right)$, que j'appelle un facteur de transfert, ressemble aux facteurs de transfert pour l'endoscopie, mais il faudra revenir encore sur cette formule, car $\Theta\left(a_{H}, a_{G}\right)$ ne sera pas nécessairement une fonction. Le produit $\Theta\left(a_{H}, a_{G}\right) d a_{G} d a_{H}$ sera plutôt une distribution en $a_{H}$ et $a_{G}$ ou une distribution stablement invariante sur le produit $H\left(F_{v}\right) \times G\left(F_{v}\right)$. Par conséquent,

$$
\left\{\int\left|\Delta\left(a_{G}\right)\right| \lambda\left(a_{G}\right) \operatorname{Orb}^{\mathrm{st}}\left(a_{G}, f^{G}\right) \Theta\left(a_{H}, a_{G}\right) d a_{G}\right\} d a_{H}
$$

sera pour chaque $f^{G}$ une distribution en $a_{H}$ qui est égale à

$$
\left|\Delta\left(a_{H}\right)\right| \lambda\left(a_{H}\right) \operatorname{Orb}^{\mathrm{st}}\left(a_{H}, f^{H}\right) d a_{H} .
$$

Nous comprendrons cette affirmation mieux en examinant par la suite le groupe $G=S L(2)$.

Le cas le plus simple est toutefois le cas où $H=\{1\},{ }^{L} H=\operatorname{Gal}(K / F)$ et $\phi$ : $\operatorname{Gal}(K / F) \rightarrow{ }^{L} G$. Alors $f^{H}$ n'est qu'une constante et cette constante est égale à

$$
\begin{aligned}
\int f^{G}(g) \chi_{\pi_{G}^{\mathrm{st}}}(g) d g & =\int\left|\Delta\left(a_{G}\right)\right| \lambda\left(a_{G}\right) \operatorname{Orb}^{\mathrm{st}}\left(a_{G}, f^{G}\right) \Theta\left(a_{H}, a_{G}\right) d a_{G} \\
& =\int \theta_{v}\left(a_{G}\right) \Theta\left(a_{H}, a_{G}\right) d a_{G}
\end{aligned}
$$

où $\theta_{v}\left(a_{G}\right)=\theta_{v}\left(a_{G} ; 1\right)$ est la fonction définie dans [FLN]. Elle est rattachée à $f^{G}$ et mérite par conséquent d'avoir un indice et d'être dénotée $\theta_{v}^{G}\left(a_{G}\right)$. Puisque nous aurons en plus de $f^{G}$ aussi une fonction $f^{H}$ nous mettrons presque toujours cet indice supplémentaire. Dans ce cas fondamental pour lequel $H=\{1\}, \Theta\left(1, a_{G}\right)=\chi_{\pi_{G}^{\text {st }}}\left(a_{G}\right)$ est donc le caractère lui-même.

Si l'on accepte de traiter la fonction $\theta^{G}=\theta_{v}^{G}$ et non la fonction $f^{G}$ comme l'objet fondamental, alors l'équation (1.1.7) devient

$$
\theta^{H}\left(a_{H}\right)=\theta_{v}^{H}\left(a_{H}\right)=\int \theta^{G}\left(a_{G}\right) \Theta\left(a_{H}, a_{G}\right) d a_{G},
$$


où $\Theta\left(a_{H}, a_{G}\right) d a_{G}$ est pour chaque $a_{H}$, ou peut-être seulement pour chaque $a_{H}$ tel que $\Delta_{H}\left(a_{H}\right) \neq 0$, une distribution en $G$. On pourrait aussi exiger que $\Theta\left(a_{H}, a_{G}\right) d a_{G} d a_{H}$ soit une distribution. Mais sans avoir caractérisé l'ensemble des $\theta^{H}$ qui sont les images des $f^{H}$ lisses à support compact on ne peut pas parler des "distributions" ni sur $\mathfrak{A}_{H}(F)$, ni sur $\mathfrak{A}_{G}(F)$, ni sur leur produit, au moins non comme des fonctions linéaires sur l'ensemble de $\theta^{H}$. Par conséquent, notre discussion restera un peu floue, mais pour le moment l'objectif principal n'est que la définition du transfert et je me permets par conséquent quelques imprécisions sur la définition de $\Theta\left(a_{H}, a_{G}\right)$. Pour la formule (1.1.3), c'est $\Theta\left(a_{H}, a_{G}\right) d a_{H}$ qui est la distribution et $a_{G}$ n'est qu'un paramètre. Tant que nous ne considérons que les sous-ensembles $\mathfrak{A}_{H}^{\mathrm{reg}}$ ou $\mathfrak{A}_{G}^{\mathrm{reg}}$, la notion d'une distribution sur les $\theta^{H}$ ou $\theta^{G}$ ne pose pas de problème. Celle d'une distribution dans le même sens sur le produit $\mathfrak{A}_{H}^{\mathrm{reg}} \times \mathfrak{A}_{G}^{\mathrm{reg}}$ est aussi évidente.

Nous ne considérons ici que le cas où $G=S L(2)$ et où $H$ est le groupe multiplicatif d'éléments de norme 1 dans une extension quadratique du corps de base. Nous admettons aussi le cas dégénéré où cette extension est déployée de sorte que $H \simeq G L(1)$. La distribution $\Theta\left(a_{H}, a_{G}\right) d a_{H} d a_{G}$ sera construite à partir de l'équation (1.1.3) Avant d'aborder les calculs, nous posons quelques questions générales.

Les caractères stables $\chi_{\pi_{G}^{\text {st }}}$ ne sont définis comme fonctions que sur l'ensemble de classes de conjugaison stables régulières semi-simples ou mieux sur son image $\mathfrak{A}_{G}^{\text {reg }}$ dans la base de Steinberg-Hitchin. En principe, dans cet ensemble, la valeur du côté gauche de (1.1.3) est connue pour $a_{G} \in \mathfrak{A}_{G}^{\mathrm{reg}}$ et pour tout $L$-paquet tempéré. Cela permettrait de trouver les valeurs $\Theta\left(a_{H}, a_{G}\right)$ pour de tels $a_{G}$, ce que nous ferons pour $S L(2)$. Supposons que $\Theta\left(a_{H}, a_{G}\right)$ soit continu comme fonction — ou distribution — stablement invariante sur le produit $H^{\mathrm{rs}}\left(F_{v}\right) \times G^{\mathrm{rs}}\left(F_{v}\right)$ des éléments réguliers et semi-simples dans $H\left(F_{v}\right)$ et dans $G\left(F_{v}\right)$. Notre première question sera:

Question A. Est-ce qu'il est possible de trouver pour chaque $f^{G}$ lisse à support compact dans l'ensemble $G^{\mathrm{rs}}\left(F_{v}\right)$ des éléments réguliers et semi-simples une fonction $f^{H}$ sur $H\left(F_{v}\right)$ lisse à support compact telle que

$$
\operatorname{Orb}^{\text {st }}\left(a_{H}, f^{H}\right)=\frac{1}{\left|\Delta\left(a_{H}\right)\right| \lambda\left(a_{H}\right)} \int\left|\Delta\left(a_{G}\right)\right| \lambda\left(a_{G}\right) \operatorname{Orb}^{\text {st }}\left(a_{G}, f^{G}\right) \Theta\left(a_{H}, a_{G}\right) d a_{G}
$$

pour tout $a_{H} \in \mathfrak{A}_{H}^{\mathrm{reg}}$ ?

Nous n'exigeons pas que le support de $f^{H}$ soit contenu dans $H^{\text {rs }}$. Une meilleure façon d'écrire l'équation (1.1.11) est l'équation (1.1.10), où on exige que $\theta_{v}^{H}\left(a_{H}\right)$ est la valeur en $a_{H}$ de la function rattachée à $f^{H}$. Implicite dans cette question est l'affirmation que pour chaque $a_{H} \in \mathfrak{S}_{H}^{\text {reg }}$ une distribution stablement invariante $\Theta\left(a_{H}, a_{G}\right) d a_{G}$ est définie. Alors

$$
\theta^{G}\left(a_{G}\right) \Theta\left(a_{H}, a_{G}\right) d a_{G}
$$

est le produit d'une fonction et de la mesure $d a_{H}$ et le problème est de trouver $f^{H}$ tel que cette fonction soit égale à $\theta_{v}^{H}$.

Une deuxième question qui se pose naturellement est la suivante.

Question B. Ayant vérifié l'existence de $f^{H}$ pour $f^{G}$ à support compact dans $G^{\mathrm{rs}}\left(F_{v}\right)$, comment pouvons-nous vérifier son existence pour $f^{G}$ à support compact dans $G\left(F_{v}\right)$ ? 
Nous traitons ces deux questions pour le groupe $G=S L(2)$ et pour les groupes $H$ diédraux, peut-être dégénérés, donc soit le groupe des éléments de norme 1 dans une extension quadratique $E$ du corps $F=F_{v}$, soit le groupe $G L(1)$. Nous abandons l'indice $v$ rattaché à $F$ pour ne l'employer que lorsque nous reviendrons au cas global. Pour $G=S L(2)$ le groupe ${ }^{L} G$ est $P G L(2)$ ou plus généralement $P G L(2) \times \operatorname{Gal}(L / F)$, avec $[L: F]$ fini. Le choix de $L$ dépend des circonstances. Si $H$ n'est pas dégéneré nous prenons $L=E$. Nous prenons alors ${ }^{L} H=G L(1) \rtimes \operatorname{Gal}(E / F), \operatorname{Gal}(E / F)=\{1, \sigma\}$, l'action de $\sigma$ étant $\sigma(x)=x^{-1}$, et l'homomorphisme ${ }^{L} H \rightarrow{ }^{L} G$ est

$$
\phi=\phi_{G, H}: x \rtimes 1 \mapsto\left(\begin{array}{cc}
x & 0 \\
0 & 1
\end{array}\right) \times 1 ; \quad 1 \rtimes \sigma \mapsto\left(\begin{array}{cc}
0 & 1 \\
1 & 0
\end{array}\right) \times \sigma .
$$

Dans le cas dégénéré on prend $L=F, \operatorname{Gal}(L / F)=\{1\}$. Le groupe ${ }^{L} H$ est $G L(1)$ et $\phi$ est donné par

$$
\phi=\phi_{G, H}: x \mapsto\left(\begin{array}{cc}
x & 0 \\
0 & 1
\end{array}\right) .
$$

Dans le cas où $G=S L(2)$, pour un corps $p$-adique et si le corps résiduel est de caractéristique positive ou pour un corps archimédien, les caractères stables ont été étudiés par Labesse-Langlands ([LL]) mais sans les expliciter. Des formules pour les caractères ont été données par Sally-Shalika. Je profite de leurs formules quoique leur article ne contient pas de démonstration. Que ces formules ne mènent pas à des difficultés est à mon avis une démonstration convaincante qu'elles ne contiennent pas d'erreur. Même avec leurs formules il n'est pas évident que l'application duale cherchée existe. C'est là l'objectif de cette première section, de comprendre quelles sont les difficultés qui rendent la construction de $f=f^{G} \mapsto f^{H}$ difficile et pourquoi les formules de Sally-Shalika nous permettent de les surmonter. Je n'ai pas essayé de démontrer l'existence du transfert stable ni pour les corps $\kappa((x))$ de séries formelles ni pour les corps de caractéristique résiduelle 2. Autrement dit, je n'ai pas essayé d'établir pour ces corps les formules de Sally-Shalika. Il est bien possible qu'elles existent déjà dans la littérature.

Les groupes $H$ qui nous intéressent étant des tores, pour leurs éléments la conjugaison stable n'est que la conjugaison — en fait les classes ne contient qu'un seul élément — et un $L$-paquet ne contient qu'une seule représentation. Rappelons qu'à chaque $H$ et chaque caractère unitaire $\pi_{H}$ de $H$ est rattachée un ensemble fini de représentations de $G$. Cet ensemble est un $L$-paquet. On le verra en citant les formules de Sally-Shalika, mais il est aussi une conséquence des principes de Labesse-Langlands, que les images $\pi_{G}^{\text {st }}$ contiennent un, deux ou quatre éléments selon les cas divers que nous décrivons par la suite. La notation pour le paquet $L$ image de $\pi_{H}$ sera $\pi_{G}^{\text {st }}$.

La représentation $\pi_{H}^{\text {st }}$ est rattachée à un homomorphisme $\phi_{H}$ du groupe de Weil $W_{L / F}$ dans ${ }^{L} H$. Dans le cas déployé, ce groupe de Weil est $F^{\times}$et $\phi_{H}=\pi_{H}$ n'est qu'un caractère $\xi$ de $F^{\times}$. On a

$$
\phi_{G}=\phi_{G, H} \circ \phi_{H}: x \mapsto\left(\begin{array}{cc}
\xi(x) & 0 \\
0 & 1
\end{array}\right) .
$$

Donc l'image n'est pas simplement abélienne, elle se relève au groupe $G L(2)$ comme groupe abélien. 
Si $H$ est vraiment tordu, alors il est rattaché à une extension quadratique $E / F$ à groupe de Galois $\{1, \sigma\}$ et $H(F)=\left\{x \in E^{\times} \mid x \sigma(x)=1\right\}$. Puisqu'il est un groupe abélien nous utilisons parfois $\xi$ pour dénoter $\pi_{H}$. Rattaché à $\xi$ est le caractère $\tilde{\xi}(x)=\xi\left(x \sigma(x)^{-1}\right)$ de $E^{\times}$. Il est convenable de prendre pour $W_{L / F}=W_{E / F}$ dans ce cas le groupe donné par une suite exacte

$$
\{1\} \rightarrow E^{\times} \rightarrow W_{E / F} \rightarrow \operatorname{Gal}(E / F) \rightarrow\{1\},
$$

où $\sigma$ se relève à $w_{\sigma}$ avec $w_{\sigma}^{2}=\alpha$ in $F^{\times}-\mathrm{N} E^{\times}$. L'homomorphisme $\phi_{H}$ est donné par $\phi_{H}(x)=\tilde{\xi}(x) \times 1, x \in E^{\times}$et $\phi_{H}\left(w_{\sigma}\right)=1 \times \sigma$. Donc $\phi_{G}=\phi_{G, H} \circ \phi_{H}$ est donné par

$$
x \in E^{\times} \mapsto\left(\begin{array}{cc}
\tilde{\xi}(x) & 0 \\
0 & 1
\end{array}\right) \times 1, \quad w_{\sigma} \mapsto\left(\begin{array}{cc}
0 & 1 \\
1 & 0
\end{array}\right) \times \sigma .
$$

Le deuxième facteur dans ces expressions est inutile et la plupart du tempsnous le supprimons.

Il est utile de réfléchir brièvement à ces conditions. La condition

$$
\phi\left(w_{\sigma}\right) \phi(x) \phi^{-1}\left(w_{\sigma}\right)=\phi(\sigma(x))
$$

équivaut à $\tilde{\xi}(\sigma(x))=\tilde{\xi}^{-1}(x)$ ou $\tilde{\xi}(s \sigma(x))=1$. La condition $\phi\left(w_{\sigma}^{2}\right)=\phi(\alpha)$ équivaut à $\tilde{\xi}(\alpha)=1$. Donc $\xi$ est trivial sur $F^{\times}$. Puisque tout élément $x$ de norme 1 dans $E^{\times}$est de la forme $y \sigma(y)^{-1}$, l'ensemble des $\tilde{\xi}$ qui interviennent est l'ensemble des caractères de $E^{\times} / F^{\times}$. L'application $\xi \mapsto \tilde{\xi}$ est une bijection entre les caractères de $H(F)$ et ceux de $E^{\times} / F^{\times}$.

Supposons que $H$ soit tordu mais que l'homomorphisme $\phi_{G}=\phi_{G, H} \circ \phi_{H}$ est aussi rattaché au groupe $G L(1)$ et à un de ces caractères. Il est ainsi si et seulement si l'image de $\phi_{G}$ se relève à un sous-groupe abélien de $G L(2)$, donc si et seulement si $\tilde{\xi}(x)=1$ pour tout $x$. Alors $\phi_{G}$ est équivalent à

$$
w \in W_{E / F} \mapsto x \in W_{F / F} \mapsto\left(\begin{array}{cc}
\eta(x) & 0 \\
0 & 1
\end{array}\right),
$$

où la première flèche est celle de la théorie du groupe de Weil et où $\eta$ est le caractère non trivial de $F^{\times} / \mathrm{N} E^{\times}$. Donc dans ce cas le paquet $\pi_{G}^{\text {st }}$ image de $\pi_{H}$ sera l'ensemble des composantes d'une représentation induite de la série principale. J'observe en passant qu'au moins pour ce cas le caractère central des éléments de $\pi_{G}^{\text {st }}$ n'est pas la restriction de $\tilde{\xi}$ à $\{ \pm 1\}$.

Une question semblable est si deux corps différents $E$ et $E^{\prime}$ et deux caractères $\xi$ et $\xi^{\prime}$ donnent, en relevant $\phi_{G}$ et $\phi_{G}^{\prime}$ de la façon habituelle à $W_{K / F}, K=E E^{\prime}$, des homomorphismes conjugués. Dans ce cas les paquets $L$ rattachés à $\{E, \xi\}$ et à $\left\{E^{\prime}, \xi^{\prime}\right\}$ sont les mêmes.

Si $\tilde{\xi}(x) \neq \pm 1$ pour tout $x \in E^{\times}$, alors le groupe de commutateurs de l'image de $W_{E / F}$ dans $P G L(2)$ est l'ensemble

$$
\left(\begin{array}{cc}
\tilde{\xi}(x) & 0 \\
0 & \tilde{\xi}^{-1}(x)
\end{array}\right)
$$


et son centralisateur est le groupe des matrices diagonales. Par conséquent, non seulement l'image inverse par rapport à $\phi_{G}$ de ce groupe dans $W_{E / F}$ est uniquement déterminée mais aussi si on relève $\phi_{G}$ à n'importe quel $W_{K / F}, E \subset K$, alors l'image inverse du groupe des matrices diagonales est $W_{K / E}$ de sorte que la classe de $\phi_{G}$ détermine $E$. Évidemment $\phi_{G}$ détermine aussi $\xi$ à un inverse près. Les deux caractères $\xi$ et $\xi^{-1}$ donnent la même $\pi_{G}^{\text {st }}$.

Si $\tilde{\xi}(x)= \pm 1$ et si les deux valeurs sont possibles, alors l'image de $\phi_{G}$ est nécessairement le groupe à quatre éléments de Klein, un sous-groupe abélien de $P G L(2)$ qui ne se relève pas à un sous-groupe de $G L(2)$ d'ordre 4 mais à un groupe d'ordre 8 . Dans ce cas, le caractère $\xi$ est d'ordre 2. L'image du groupe $W_{E / F}$ étant d'ordre 4, elle définit un quotient, le groupe de Galois $\operatorname{Gal}(K / F)$ d'une extension galoisienne $K / F$ de degré 4 qui contient trois extensions quadratiques de $F$ dont l'une est $E$. Si $E^{\prime}$ et $E^{\prime \prime}$ sont les autres et si $H^{\prime}$ et $H^{\prime \prime}$ sont les tores qui leur correspondent, alors il est évident que $\phi_{G}$ et la classe stable (le $L$-paquet) $\pi_{G}^{\text {st }}$ y rattachée peuvent être aussi définis à partir de caractères $\xi^{\prime}$ et $\xi^{\prime \prime}$ de $H^{\prime}(F)$ et $H^{\prime \prime}(F)$. Comme expliqué dans [LL], cette classe contient quatre éléments.

1.2. Le tore déployé. Le cas le plus simple est celui où $H=G L(1)$ ou le tore déployé de SL(2). C'est le cas le plus simple et il peut être traité d'une façon uniforme pour les trois corps locaux qui interviennent: le corps des nombres complexes; le corps des nombres réels; et les corps $p$-adiques. On peut aussi y ajouter les corps des séries formelles, mais ce n'est guère la peine en ce moment. Soit $\pi_{H}=\xi$. Le caractère stable $\pi_{G}^{\text {st }}$ est une distribution, mais elle est donnée par une function stablement invariante sur les éléments réguliers et semi-simples. Cette fonction est 0 sur les classes elliptiques mais sur les classes déployées, dont chacune est donnée par une matrice diagonale

$$
\gamma=\gamma(t)=\left(\begin{array}{cc}
t & 0 \\
0 & t^{-1}
\end{array}\right)
$$

le caractère est donné par

$$
\chi_{G}^{\mathrm{st}}(\gamma)=\frac{\xi(t)+\xi^{-1}(t)}{\left|\Delta_{G}(\gamma)\right|}
$$

où la fonction $\Delta_{G}(\gamma)= \pm \sqrt{\left(1-t^{2}\right)\left(1-t^{-2}\right)}=\Delta_{G}\left(a_{G}\right), a_{G}=\mathbf{c}(t)$, est celle introduite dans [FLN]. Puisque le groupe $H$ est un tore, le caractère de $\pi_{H}$ est simplement $\xi$. La fonction $\Delta_{H}$ est identiquement 1.

Grâce à la formule (1.2.1) la distribution $\Theta\left(a_{H}, a_{G}\right) d a_{G} d a_{H}$ sera une distribution concentrée en $\gamma_{G}=\gamma_{H}^{ \pm 1}$. Pour nous exprimer précisément, rappelons que la base de SteinbergHitchin pour $H$ est $H$ lui-même tandis que la base pour $G$ est la ligne droite de sorte que $a_{H}=t=t_{H}$ et $a_{G}=\mathbf{c}(t)=t+t^{-1}=t_{G}+t_{G}^{-1}$. La notation $t$ est plus agréable, et nous l'utilisons autant que possible, mais les notations $t_{H}, t_{G}$ admettent la possibilité que $t_{G} \neq t_{H}$ et sont donc souvent indispensables. Pour le cas particulier qui est étudié dans cet article, $\lambda\left(a_{H}\right)=1$ et $\lambda\left(a_{G}\right)=L\left(1, \sigma_{T_{H}}\right)$, si $T_{H}$ est un tore dans $G$ isomorphe à $H$. De plus,

$$
d a_{H}=L\left(1, \sigma_{H}\right) d^{\times} t=L\left(1, \sigma_{H}\right) \frac{d t}{t} .
$$


Par contre,

$$
d a_{G}=\left|1-t^{-2}\right| d t=\left|\Delta_{G}(t)\right| \frac{d t}{t}=\frac{\left|\Delta_{G}\left(a_{G}\right)\right|}{L\left(1, \sigma_{H}\right)} d a_{H} .
$$

Selon la formule (1.1.3) dont le côté gauche est donné dans ce cas par (1.2.1), la distribution $\Theta\left(a_{H}, a_{G}\right) d a_{H}$, qui rattache à chaque fonction sur $\mathfrak{A}_{H}(F)=H(F)$ une fonction sur $\mathfrak{A}_{G}^{\mathrm{rs}}(F)$ ou sur les classes de conjugaison stable dans $G^{\mathrm{rs}}(F)$, est une somme de deux fonctions du type $\delta$,

$$
\Theta\left(a_{H}, a_{G}\right) d a_{H}=\frac{\delta_{t_{G}}\left(t_{H}\right)+\delta_{t_{G}^{-1}}\left(t_{H}\right)}{\left|\Delta_{G}\left(a_{G}\right)\right|} .
$$

Donc dans ce cas au moins, l'expression au côté gauche est effectivement une distribution. Mais cette expression est mauvaise du point de vue de la notation. L'application $a_{H} \mapsto a_{G}$ est de degré deux. La distribution donnée par le produit de (1.2.3) et de $d a_{G}$ est la distribution qui rattache à une fonction $h\left(a_{H}, a_{G}\right)$ la valeur

$$
\int h\left(a_{H}, a_{G}\right) \Theta\left(a_{H}, a_{G}\right) d a_{H} d a_{G}
$$

où l'intégrale se fait sur $\mathfrak{A}_{G}(F)$ — ou plutôt sur l'ensemble des $a_{G}$ donnés par les éléments de $G^{\text {sp }}$ qui sont réguliers, semi-simples et déployés, donc conjugués à un élément de $T_{H}$. Plus précisement, elle lui rattache la valeur

$$
\int\left(\sum_{a_{H} \mapsto a_{G}} \frac{h\left(a_{H}, a_{G}\right)}{\left|\Delta_{G}\left(a_{G}\right)\right|}\right) d a_{G}=\int\left(\frac{h\left(a_{H}, a_{G}\right)}{L\left(1, \sigma_{H}\right)}\right) d a_{H},
$$

où à droite aussi bien qu'à gauche $a_{H} \mapsto a_{G}$. Donc l'objet que nous avons dénoté $\Theta\left(a_{H}, a_{G}\right) d a_{G} d a_{A}$ et appelé une distribution doit être maniée avec quelque circonspection.

Pour les paires que nous étudions, l'expression (1.1.9) n'est que $f^{H}\left(t_{H}\right) d a_{H}$. Avec la distribution donnée par les formules (1.2.3), (1.2.4) et (1.2.5), la formule (1.1.10) donne

$$
f^{H}\left(t_{H}\right)=\theta^{H}\left(a_{H}\right)=\frac{\theta^{G}\left(a_{G}\right)}{L\left(1, \sigma_{H}\right)}=\frac{\theta^{G}\left(a_{G}\right)}{\lambda\left(a_{G}\right)}, \quad a_{H} \mapsto a_{G}
$$

Rappelons que $L\left(1, \sigma_{H}\right)=1 /(1-1 / q)$, où $q$ est le nombre d'éléments dans le corps résiduel. En plus, si $a_{H} \mapsto a_{G}$, alors $\lambda\left(a_{G}\right)=L\left(1, \sigma_{H}\right)$. Ce dénominateur se trouve comme facteur dans la définition de $\theta^{G}\left(a_{G}\right)$. La formule (1.2.6) définit $f^{H}$ aux points réguliers de $H(F)$. Les lemmes 2.1.2, 2.1.3 et 2.1.4 nous permettent de le définir comme fonction lisse à support compact partout sur $H(F)$.

1.3. Le tore elliptique sur le corps réel. Le groupe de Weil pertinent est l'extension du groupe $\operatorname{Gal}(\mathbb{C} / \mathbb{R})$ par le groupe $\mathbb{C}^{\times}$et les homomorphismes $\phi_{H}$ sont donnés par $z \mapsto$ 
$z^{m} \bar{z}^{-m} \rtimes 1, z \in \mathbb{C}, w_{\sigma} \mapsto 1 \rtimes \sigma$. L'ensemble $H(F), F=\mathbb{R}$, est l'ensemble des nombres complexes de valeur absolue 1 . Le paquet $\pi_{H}^{\text {st }}$ rattaché à $\phi_{H}$ ne contient qu'une seule représentation, à savoir le caractère $z \mapsto z^{m},|z|=1$. Le cas $m=0$ est dégénéré et l'homomorphisme $\phi_{G}=\phi_{G, H} \circ \phi_{H}$ correspond alors aux composantes d'une représentation de la série principale. Si $m \neq 0$ le $L$-paquet rattaché à lui est le même pour $-m$ que pour $m$ et contient deux éléments de la série discrète. Le caractère stable de ce paquet est bien connu. Comme référence je renvoie le lecteur au livre de Knapp ([Kn]) en lui conseillant de faire attention à la définition de $a_{t}$ y donnée, car il est parfois le $a(t)$ de la formule (1.3.1), parfois $a(t / 2)$. J'utilise ses calculs mais avec $a_{t}=a(t)$. Avec $\chi_{m}=\chi_{\pi}^{\text {st }}, m \geq 0$, ils donnent,

$$
\begin{aligned}
\chi_{m}( \pm a(t)) & =( \pm 1)^{m-1} \frac{2 e^{-m|t|}}{e^{t}-e^{-t}}, & \pm a(t) & = \pm\left(\begin{array}{cc}
e^{t} & 0 \\
0 & e^{-t}
\end{array}\right), \quad t>0, \\
\chi_{m}(s(\theta)) & =-\frac{e^{i|m| \theta}-e^{-i|m| \theta}}{e^{i \theta}-e^{-i \theta}}, & s(\theta) & =\left(\begin{array}{cc}
\cos \theta & \sin \theta \\
-\sin \theta & \cos \theta
\end{array}\right) .
\end{aligned}
$$

Pour $m=0$, ce caractère est zéro sur le tore compact et égal à $2 \operatorname{sgn}\left( \pm e^{t}\right) /|\Delta( \pm a(t))|$ sur le tore déployé, donc égal à (1.2.1) pour $\xi$ égal au caractère $\xi: t \mapsto \operatorname{sgn}(t)$.

Il y a un conflit de notation entre cet article et le livre de Knapp. Dans cet article, au moins pour le moment, $t$ est une des deux valeurs propres d'une matrice diagonale dans $S L(2)$. Dans le livre de Knapp cette valeur propre est $\pm a(t)$. Il serait d'un côté incommode de modifier la notation de cet article et de la remplacer par celle de Knapp. De l'autre côté, celle de Knapp nous est donnée. Donc lorsqu'il s'agit des calculs basés sur les renvois à son livre $[\mathrm{Kn}]$, j'utilise sa notation et j'évite celle autrement employée ici. Les sections pertinentes sont celle-ci aussi bien que $\S 2.1$.

Nous calculons les facteurs de transfert de $G$ à $H$ d'abord sur le tore compact dans $G$ et ensuite sur le tore déployé, en commençant en même temps par la vérification que les réponses aux questions $\mathrm{A}$ et $\mathrm{B}$ sont affirmatives. Soient $\theta_{H}$ et $\theta_{G}$ les paramètres dans $H$ et $G$ respectivement. Il résulte de (1.3.1) et des définitions que le développement de Fourier de $\Theta\left(s\left(\theta_{H}\right), s\left(\theta_{G}\right)\right) d a_{H}$ est

$$
-\sum_{m} \frac{e^{i|m| \theta_{G}}-e^{-i|m| \theta_{G}}}{e^{i \theta_{G}}-e^{-i \theta_{G}}} e^{-i m \theta_{H}} d a_{H} .
$$

On a

$$
\Delta^{2}\left(s\left(\theta_{G}\right)\right)=\left|1-\exp \left(2 i \theta_{G}\right)\right|\left|1-\exp \left(-2 i \theta_{G}\right)\right|=\left|e^{i \theta_{G}}-e^{-i \theta_{G}}\right|^{2} .
$$

Les équations (1.2.2) et (1.2.5) sont valables et nécessaires aussi pour le tore compact.

Considérons la question A en supposant que $f^{G}$ lui-même est à support dans l'ensemble des éléments elliptiques réguliers. Dans le cas que nous traitons à présent $\left|\Delta\left(a_{H}\right)\right|=1$. Puisque le produit $\lambda\left(a_{H}\right) L\left(1, \sigma_{H}\right)=1$, le côté droit de (1.1.7) est, grâce à (1.2.5),

$$
\int\left|\Delta\left(a_{G}\right)\right|^{2} \lambda\left(a_{G}\right) \operatorname{Orb}^{\mathrm{st}}\left(a_{G}, f^{G}\right) \Theta\left(a_{H}, a_{G}\right) d \theta_{G} .
$$


Nous sommes en danger de nous perdre dans la notation et dans les notions: distribution ou fonction; le groupe $G$ ou le groupe $H$; mesures et intégrales sur $G(F)$ et $H(F)$ ou sur leurs bases de Steinberg-Hitchin. Le meilleur choix n'est pas toujours évident. L'expression (1.3.2) n'est encore qu'une distribution sur $H(F)$ qui, en fonction symétrique de $\theta_{G}$, ne dépend que de $a_{G}$. Nous ne savons pas encore qu'elle est, comme distribution en $a_{H}$, donnée par une fonction, car il n'est pas évident que la série (1.3.2) converge. Nous devons pour le moment multiplier l'expression au côté droit de (1.1.11) par $d a_{H}$. Étant donné la forme de (1.3.2), il est mieux d'employer pour l'intégration la variable $\theta_{G}$ plutôt que la variable $a_{G}$. Cela introduit un facteur $\left|\Delta\left(a_{G}\right)\right|$ supplémentaire qui est inclu dans la formule (1.3.4). Ceci expliqué, nous revenons à (1.3.4) qui donne lorsque nous le multiplions par $d a_{H}$ la distribution,

$$
\lambda\left(a_{G}\right)\left(e^{i \theta_{G}}-e^{-i \theta_{G}}\right) \operatorname{Orb}^{\mathrm{st}}\left(a_{G}, f^{G}\right) \sum_{m}\left(e^{i|m| \theta_{G}}-e^{-i|m| \theta_{G}}\right) e^{-i m \theta_{H}} d a_{H} .
$$

Nous écartons pour le moment le facteur

$$
\lambda\left(a_{G}\right)\left(e^{i \theta_{G}}-e^{-i \theta_{G}}\right) \operatorname{Orb}^{\mathrm{st}}\left(a_{G}, f^{G}\right)
$$

qui ne dépend que de $\theta_{G}$ pour examiner la distribution

$$
\sum_{m}\left(e^{i|m| \theta_{G}}-e^{-i|m| \theta_{G}}\right) e^{-i m \theta_{H}} d a_{H}
$$

Le facteur et la distribution sont tous les deux des fonctions impaires de $\theta_{G}$, mais nous n'en profiterons pas pour simplifier la distribution. Puisque nous n'abordons pour le moment que la question $A$, nous supposons que $\theta_{G} \neq 0, \pi$ modulo $2 \pi$. La distribution est certainement la limite des distributions données par des fonctions,

$$
\lim _{z \nearrow 1} \sum_{m}\left(z^{|m|} e^{i|m| \theta_{G}}-z^{|m|} e^{-i|m| \theta_{G}}\right) e^{-i m \theta_{H}}
$$

Pour cette somme il y a une formule close,

$$
\frac{z e^{i \theta_{G}-i \theta_{H}}-z e^{-i \theta_{G}+i \theta_{H}}}{\left(1-z e^{i \theta_{G}-i \theta_{H}}\right)\left(1-z e^{-i \theta_{G}+i \theta_{H}}\right)}+\frac{z e^{i \theta_{G}+i \theta_{H}}-z e^{-i \theta_{G}-i \theta_{H}}}{\left(1-z e^{i \theta_{G}+i \theta_{H}}\right)\left(1-z e^{-i \theta_{G}-i \theta_{H}}\right)} .
$$

Si $\theta_{H}^{\prime} \not \equiv \pm \theta_{G}(\bmod 2 \pi \mathbb{Z})$ nous pouvons passer à $z=1$ pour obtenir une distribution qui dans un voisinage de $\theta_{H}^{\prime}$ est donnée par la fonction

$$
\frac{e^{i \theta_{G}-i \theta_{H}}-e^{-i \theta_{G}+i \theta_{H}}}{\left(1-e^{i \theta_{G}-i \theta_{H}}\right)\left(1-e^{-i \theta_{G}+i \theta_{H}}\right)}+\frac{e^{i \theta_{G}+i \theta_{H}}-e^{-i \theta_{G}-i \theta_{H}}}{\left(1-e^{i \theta_{G}+i \theta_{H}}\right)\left(1-e^{-i \theta_{G}-i \theta_{H}}\right)} .
$$

Puisque $f^{G}$ est à support compact dans $G^{\mathrm{rs}}(F)$, la fonction (1.3.5) a son support dans l'ensemble $\left\{\theta_{G} \not \equiv 0, \pi(\bmod 2 \pi)\right\}$ et elle est lisse. Rappelons qu'une réponse affirmative à la 
question A n'exige pas que (1.3.6) soit une distribution donnée par une fonction lisse, mais que l'intégrale par rapport à $d a_{G}$ de son produit avec (1.3.5) soit une distribution donnée par une fonction lisse de $a_{H}$. Notre argument se développe en deux étapes. Nous examinons d'abord, comme distribution en la variable $\theta_{H}$, la limite (1.3.8) des distributions (1.3.7) pour un $\theta_{G} \not \equiv 0, \pi(\bmod 2 \pi)$. Cette limite ne sera pas d'abord une distribution donnée par une fonction lisse. Mais en la multipliant par une fonction lisse de $\theta_{G}$ à support dans $\left\{\theta_{G} \not \equiv 0(\bmod \pi)\right\}$ et en prenant l'intégrale par rapport à $d a_{G}$, elle devient une distribution donnée par le produit de $d a_{H}$ et d'une fonction lisse de $\theta_{H}$. Cette fonction ne sera pas toutefois une distribution à support dans $\left\{\theta_{H} \not \equiv 0(\bmod \pi)\right\}$ !

Pour la démonstration, nous pouvons supposer que (1.3.5) est une fonction lisse à support dans un petit voisinage d'un $\theta_{G}^{\prime} \not \equiv 0, \pi(\bmod 2 \pi)$, mais autrement arbitraire. Nous traitons les deux termes de (1.3.8) séparément, ou plutôt nous ne traitons que le premier, en travaillant dans un voisinage de $\theta_{H}=\theta_{G}^{\prime}, \theta_{G}=\theta_{G}^{\prime}$. Soit $x=\theta_{H}-\theta_{G}$. Soit $g=g\left(\theta_{H}\right)$ la fonction de $\theta_{H}$ en laquelle nous évaluons le premier terme de la distribution (1.3.8) et sa limite, le premier terme de (1.3.9),

$$
\frac{e^{i \theta_{G}-i \theta_{H}}-e^{-i \theta_{G}+i \theta_{H}}}{\left(1-e^{i \theta_{G}-i \theta_{H}}\right)\left(1-e^{-i \theta_{G}+i \theta_{H}}\right)}=\frac{2}{i x}+h_{1}(x),
$$

où $h_{1}$ est une fonction lisse dans un voisinage de $x=0$.

Pour nous préparer pour les calculs précis et pour la vérification de l'existence de la limite, définissons la distribution rattachée au quotient $2 / i x$. Écrivons $g\left(x+\theta_{G}\right)=a+h(x)$, $h(0)=0$. La distribution sera

$$
\begin{aligned}
\frac{2}{i} \lim _{\epsilon \rightarrow 0} \int_{\epsilon \leq|x| \leq \pi} \frac{g(x)}{x} d x & =\frac{2}{i} \lim _{\epsilon \searrow 0}\left\{\int_{-\pi}^{-\epsilon}+\int_{\epsilon}^{\pi}\right\} \frac{a}{x} d x+\frac{2}{i} \int_{-\pi}^{\pi} \frac{h(x)}{x} d x \\
& =\frac{2}{i} \int_{-\pi}^{\pi} \frac{h(x)}{x} d x .
\end{aligned}
$$

Nous écrivons

$$
\frac{2}{i} \int_{-\pi}^{\pi} \frac{h(x)}{x} d x=\frac{2}{i} \int_{-\pi}^{0} \frac{h(x)}{x} d x+\frac{2}{i} \int_{0}^{\pi} \frac{h(x)}{x},
$$

pour ensuite remplacer les limites 0 par $\pm \epsilon$, ce qui donne, après une intégration partielle,

$$
\frac{2}{i}\{h(x) \ln |x|\}_{-\pi}^{-\epsilon}+\frac{2}{i}\{h(x) \ln |x|\}_{\epsilon}^{\pi}+2 i\left\{\int_{-\pi}^{-\epsilon}+\int_{\epsilon}^{\pi}\right\} h^{\prime}(x) \ln |x| d x .
$$

En passant à la limite $\epsilon=0$, nous obtenons

$$
2 i \int_{-\pi}^{\pi} h^{\prime}(x) \ln |x| d x=2 i \int_{-\pi}^{\pi} g^{\prime}\left(\theta_{H}\right) \ln \left|\theta_{H}-\theta_{G}\right| d \theta_{H} .
$$

Nous vérifions maintenant que la limite des distributions (1.3.7) existe et que, lorsqu'on tient compte du terme $h_{1}(x)$ dans (1.3.10) et du seconde terme de (1.3.9) ou (1.3.10), elle est égale à

$$
2 i \int_{-\pi}^{\pi}\left\{\ln \left|e^{i\left(\theta_{H}-\theta_{G}\right)}-1\right|-\ln \left|e^{i\left(\theta_{H}+\theta_{G}\right)}-1\right|\right\} g^{\prime}\left(\theta_{H}\right) d \theta_{H}
$$


Observons que les deux fonctions $\ln \left|\theta_{H}-\theta_{G}\right|$ et $\ln \left|e^{i\left(\theta_{H}-\theta_{G}\right)}-1\right|$ ont la même singularité à $\theta_{H}=\theta_{G}$. Il faudra ensuite vérifier qu'en intégrant le produit de cette distribution avec une fonction lisse de $\theta_{G}$ à support qui ne contient ni 0 ni $\pi$ nous obtenons une distribution en $a_{H}$ donnée par une fonction lisse. Il est cependant bien possible que le point 0 ou le point $\pi$ soit contenu dans le support de cette fonction.

Pour un $a_{G} \not \equiv 0$ et un $\pi$ donné, le premier terme de la distribution rattachée à (1.3.8) est

$$
z \int_{-\pi}^{\pi} \frac{e^{-i x}-e^{i x}}{\left(1-z e^{-i x}\right)\left(1-z e^{i x}\right)} g\left(x+\theta_{G}\right) d x
$$

où la fonction est comme ci-dessus la fonction en laquelle la distribution est évaluée. Cette expression est égale à

$$
\int_{-\pi}^{\pi} \frac{1}{1-z e^{-i x}} g\left(x+\theta_{G}\right) d x-\int_{-\pi}^{\pi} \frac{1}{1-z e^{i x}} g\left(x+\theta_{G}\right) d x .
$$

La première de ces deux intégrales est égale à

$$
\left\{-i \ln \left(e^{i x}-z\right) g\left(x+\theta_{G}\right)\right\}+i \int_{-\pi}^{\pi} \ln \left(e^{i x}-z\right) g^{\prime}\left(x+\theta_{G}\right) d x
$$

la deuxième, le signe y compris, est

$$
\left\{-i \ln \left(e^{-i x}-z\right) g\left(x+\theta_{G}\right)\right\}+i \int_{-\pi}^{\pi} \ln \left(e^{-i x}-z\right) g^{\prime}\left(x+\theta_{G}\right) d x .
$$

Pour $0<z<1$, la fonction $\ln \left(e^{i x}-z\right)$ change par $2 \pi i$ lorsque $x$ passe de $-\pi$ à $\pi$ tandis que $\ln \left(d^{-i x}-z\right)$ change par $-2 \pi i$. Puisque $g\left(\pi+\theta_{G}\right)=g(-\pi+\theta)$, les premiers termes dans ces deux expressions s'annulent mutuellement. Ce qui reste de leur somme est

$$
i \int_{-\pi}^{\pi} \ln \left|e^{i x}-z\right|^{2} g^{\prime}\left(x+\theta_{G}\right) d x
$$

qui converge vers

$$
2 i \int_{-\pi}^{\pi} \ln \left|e^{i x}-1\right| g^{\prime}\left(x+\theta_{G}\right) d x
$$

lorsque $z \rightarrow 1$. Le deuxième terme de (1.3.8) se traite d'une façon semblable.

Il reste à vérifier qu'en multipliant (1.3.11) avec une fonction $f_{1}$ lisse de $\theta_{G}$ dont le support ne contient ni 0 ni $\pi$ et en intégrant par rapport à $d \theta_{G}$ nous obtenons une distribution périodique de $\theta_{H}$ donnée partout par une fonction lisse. Observons que dans le domaine $\theta_{G} \not \equiv 0, \pi(\bmod \pi)$ la distribution $f_{1}\left(\theta_{G}\right) d \theta_{G}$ est aussi de la forme $f_{2}\left(a_{G}\right) d a_{G}$, où $f_{2}$ est aussi lisse que $f_{1}$. 
La distribution (1.3.11) est, en fonction de $\theta_{G}$, impaire. Donc nous pouvons supposer que $f_{1}$ est aussi impair, ce qui est de toute façon le cas pour les fonctions qui interviennent, celles de (1.3.5). Cela donne, en posant $\theta=\theta_{G}$,

$$
\int_{-\pi}^{\pi} f_{1}(\theta) \int_{-\pi}^{\pi}\left\{\ln \left|e^{i\left(\theta_{H}-\theta\right)}-1\right|-\ln \left|e^{i\left(\theta_{H}+\theta\right)}-1\right|\right\} g^{\prime}\left(\theta_{H}\right) d \theta_{H} d \theta
$$

Il suffit de montrer que la distribution en $\theta_{H}$,

$$
\int_{-\pi}^{\pi} f_{1}(\theta) \int_{-\pi}^{\pi}\left(\ln \left|e^{i\left(\theta_{H}-\theta\right)}-1\right|\right) g^{\prime}\left(\theta_{H}\right) d \theta_{H} d \theta
$$

est donnée par une fonction lisse.

Cette distribution est donné par

$$
\int_{-\pi}^{\pi} f_{2}\left(\theta_{H}\right) g^{\prime}\left(\theta_{H}\right) d \theta_{H}
$$

où $f_{2}$ est le produit de convolution

$$
f_{2}\left(\theta_{H}\right)=\int_{-\pi}^{\pi} f_{1}(\theta) \ln \left|e^{i\left(\theta_{H}-\theta\right)}-1\right| d \theta
$$

Si $\left\{a_{n}\right\}$ et $\left\{b_{n}\right\}$ sont les coefficients de Fourier des deux facteurs d'un tel produit, alors les coefficients du produit sont $2 \pi a_{n} b_{n}$, ce qui rend, en particulier, évident que le produit d'une fonction lisse et d'une fonction intégrable est lisse. La fonction $\ln \left|e^{i(\theta)}-1\right|$ est intégrable. Puisque (1.3.18) est aussi égal à

$$
-\int_{-\pi}^{\pi} f_{2}^{\prime}\left(\theta_{H}\right) g\left(\theta_{H}\right) d \theta_{H}
$$

la distribution (1.3.17) est donnée par une fonction lisse.

Si le support de $f^{G}$ est contenu dans l'ensemble de éléments déployés et réguliers, la démonstration de l'existence de $f^{H}$ est beaucoup plus simple. Selon la première des équations (1.3.1), le développement de Fourier de $\Theta\left(s\left(\theta_{H}\right), \pm a\left(t_{G}\right)\right), t_{G}>0$, est

$$
2 \sum_{m=-\infty}^{\infty}( \pm 1)^{m-1} \frac{e^{-|m| t_{G}}}{e^{t_{G}}-e^{-t_{G}}} e^{-i m \theta_{H}} .
$$

Nous calculons le côté droit de (1.1.7) comme pour le tore compact, mais en remplaçant $d \theta_{H}$ par $d t_{H} / t_{H}$. Observons que $d \theta_{H}$ donne la même mesure que $d s / s, s=e^{i \theta_{H}}$. Malheureusement, devant l'alternative, mesure sur le tore ou mesure sur la base de Steinberg-Hitchin, le meilleur choix n'est pas toujours le même. Il faut faire attention.

Puisque

$$
\left|\Delta\left( \pm a\left(t_{G}\right)\right)\right|^{2}=\left(e^{t_{G}}-e^{-t_{G}}\right)^{2}
$$


nous obtenons

$$
2\left(e^{t_{G}}-e^{-t_{G}}\right) \sum_{m}( \pm 1)^{m-1} e^{-|m| t_{G}} e^{-i m \theta_{H}} .
$$

Il est évident que cette expression définit, lorsqu'elle est multipliée par une fonction lisse de $\pm a\left(t_{G}\right)$ qui s'annule dans un voisinage de $\pm a(0)$ et intégrée sur le tore déployé, une fonction lisse de $\theta_{H}$. La réponse à la question $\mathrm{A}$ est donc affirmative pour le corps $\mathbb{R}$. Pour répondre à la question $\mathrm{B}$, nous aurons besoin des résultats de la partie $\S 2.1$. Avant de passer à cette deuxième question écrivons d'une façon plus précise, les formules pour le tore déployé. Si on prend $t_{G}>0$ l'expression (1.3.5) vaut $\theta^{G}\left(a_{G}\right)$ et (1.3.6) devient

$$
2 \sum_{m}( \pm 1)^{m-1} e^{-|m| t_{G}} e^{-i m \theta_{H}} .
$$

Pour définir la fonction $f^{H}$ rattachée à un $f^{G}$ général il faut approximer $f^{G}$ par des fonctions $\tilde{f}^{G}$ lisses et à support dans l'ensemble $G^{\mathrm{rs}}(F)$. Cette approximation sera nécessairement faible. On peut exiger, par exemple, que le support de $f_{1}^{G}$ reste dans un ensemble compact et que

$$
\int\left|\tilde{f}^{G}(g)-f^{G}(g)\right| d g \rightarrow 0
$$

et que, en plus, la distribution $\tilde{f}^{H}$ converge vers $f^{H}$, donc que chacun de ses coefficients de Fourier convergent vers le coefficient correspondant de $f^{H}$. Pourvu que ces limites ne dépendent pas du choix de la suite des fonctions $\tilde{f}$, cette définition de $f^{H}$ est tout à fait convenable. Il faut toutefois vérifier l'existence de ces limites et de leur unicité. Il faut aussi montrer que la distribution limite est donnée par une fonction lisse.

Soit $\tilde{\theta}^{G}=\theta_{\tilde{f}}^{G}$. Il résulte de (1.3.23) et de la formule (3.31) de [FLN] que

$$
\int_{\mathfrak{A}(\mathbb{R})}\left|\tilde{\theta}^{G}\left(a_{G}\right)-\theta^{G}\left(a_{G}\right)\right| d a_{G} \rightarrow 0 .
$$

Lorsque le support de $\tilde{f}$ est contenu dans $G^{\mathrm{rs}}(\mathbb{R})$ la distribution sur $H(\mathbb{R})$ y rattachée est la somme de deux distributions, l'une rattachée à la restriction de $\tilde{f}$ aux éléments elliptiques, l'autre à sa restriction aux éléments déployés. Il faut les calculer séparément. Il s'avère que la limite des deux lorsque $\tilde{f}^{G} \rightarrow f^{G}$ existent mais qu'en général ni l'une ni l'autre des limites seront des distributions données par des fonctions. Leur somme l'est cependant. Le second des deux prochains lemmes nous permet de le vérifier.

Lemme 1.3.1. Soit $\phi$ une fonction périodique de période $2 \pi$ sur la ligne droite, localement intégrable et lisse partout sauf en des points $x_{1}, \ldots, x_{n}$ modulo $\pi$ mais on exige qu'en ces points ses dérivées $\phi^{(k)}, k \geq 0$, ont des limites à droite et à gauche. Soit

$$
\phi^{(k)}\left(x_{j}^{+}\right)-\phi^{(k)}\left(x_{j}^{+}\right)=\alpha_{j}^{(k)} .
$$


Soient

$$
c_{m}=\frac{1}{2 \pi} \int_{0}^{2 \pi} \phi(\theta) e^{i m \theta} d \theta
$$

les coefficients de Fourier de $\phi$. Alors pour tout entier $K \geq 0$,

$$
c_{m}-\frac{1}{2 \pi} \sum_{j=1}^{n} \sum_{k=0}^{K-1} \frac{i^{k+1} \alpha_{j}^{(k)}}{m^{k+1}} e^{i m x_{j}}=O\left(m^{-K-1}\right),
$$

la majoration étant uniforme en $m$ pour un $K$ donné.

Lemme 1.3.2. Supposons que, pour un choix convenable des constantes $\alpha_{j}^{(k)}$, les coefficients de Fourier d'une distribution satisfont à (1.3.24) pour tout K. Alors cette distribution est donnée par une fonction $\phi$ qui est lisse à part des sauts $\alpha_{j}^{(k)}$ de ses dérivées aux points $x_{j}$.

Le premier de ces lemmes est certainement correct pour $\phi$ lisse. Il suffit alors de le vérifier pour $n=1, x_{1}=0$, et pour une fonction dont le support est contenu dans un petit voisinage de $x_{1}$. Nous avons

$$
\begin{aligned}
2 \pi c_{m}=\int_{-\pi}^{\pi} \phi(\theta) e^{i m \theta} d \theta & =\int_{-\epsilon}^{0} \phi(\theta) e^{i m \theta} d \theta+\int_{0}^{\epsilon} \phi(\theta) e^{i m \theta} d \theta \\
& =\frac{i}{m} \phi\left(0^{-}\right)-\frac{1}{i m} \phi\left(0^{+}\right)-\frac{1}{i m} \int_{-\epsilon}^{\epsilon} \phi^{\prime}(\theta) e^{i m \theta} \\
& =\frac{i}{m} \alpha_{1}^{(0)}+\frac{i}{m} \int_{-\epsilon}^{\epsilon} \phi^{\prime}(\theta) e^{i m \theta} d \theta
\end{aligned}
$$

La preuve se poursuit par récurrence. Pour le deuxième lemme, nous pouvons sans difficulté construire une fonction $\psi$ lisse en dehors de $\left\{x_{1}, \ldots, x_{n}\right\}$ et dont les dérivées ont les sauts $\alpha_{j}^{(k)}$ en ces points jusqu'à n'importe quel degré $K$ donné, mais dont les sauts des dérivées de degré plus élevé ne sont pas prescrits. Il résulte du lemme 1.3.1 que l'ordre de grandeur des coefficients $c_{m}$ de Fourier de la fonction $\phi-\psi$ est $O\left(m^{-K-1}\right)$, de sorte que les dérivées de $\phi-\psi$ jusqu'au degré $K-1$ sont continues. Puisque $K$ est arbitraire le lemme s'ensuit.

L'expression (1.3.5) est égale à

$$
i \operatorname{sgn}\left(\sin \theta_{G}\right) \theta_{f^{G}}^{G}\left(a_{G}\right) .
$$

La distribution rattachée à la fonction $\tilde{f}^{G}$ est la somme des distributions rattachées à ses restrictions à l'ensemble des éléments elliptiques et réguliers et à l'ensemble des éléments déployés et réguliers. La première est donnée par (1.3.16) avec

$$
f_{1}(\theta)=i \operatorname{sgn}(\sin \theta) \theta_{\tilde{f}^{G}}^{G}\left(a_{G}\right)
$$

Il est donc évident que sa limite, lorsque $\tilde{f}^{G} \rightarrow f^{G}$, est donnée par (1.3.16) mais avec

$$
f_{1}(\theta)=i \operatorname{sgn}(\sin \theta) \theta_{f^{G}}^{G}\left(a_{G}\right)
$$


Il s'agit de la différence de deux produits de convolution, pas nécessairement de fonctions mais de distributions,

$$
f_{1} * \delta-f_{2} * \delta
$$

où $f_{2}(\theta)=f_{1}(-\theta)$ et

$$
(\delta, g)=\int_{-\pi}^{\pi} \ln \left|e^{i \theta_{H}}-1\right| g^{\prime}\left(\theta_{H}\right) d \theta_{H}
$$

Puisque $f_{1}$ est supposé être impair $f_{1} * \delta-f_{2} * \delta=2 f_{1} * \delta$.

Soient $c_{m}$ les coefficients de Fourier de $2 f_{1}$ et $d_{m}$ ceux de $\delta$ de sorte que ceux de $2 f_{1} * \delta$ sont $2 \pi c_{m} d_{m}$. La distribution $\delta$ est la dérivée d'une fonction intégrable. Donc $d_{m} / m=O(1)$ et les coefficients de Fourier de sa convolution avec une fonction dont l'ordre de grandeur des coefficients de Fourier est $O\left(1 /|m|^{K}\right)$ pour tout $K>0$, donc une fonction lisse, ont cette même propriété. La fonction $f_{1}$ n'a des singularités qu'à $\theta=0, \pi$. Selon le lemme 2.1.4, $\theta_{f G}^{G}=\theta_{f}^{G}$ est dans un voisinage de $\theta_{0}=0$, $\pi$, donc aux points $a_{G}= \pm(2+y)$, $y<0$, la somme de deux fonctions, $\theta_{e}^{G}$ et $\theta_{o}^{G}$. Selon la formule (2.1.7), la fonction $\theta_{o}^{G}$ a, comme fonction de $\theta_{G}=\theta_{0}+\theta$, un développement formel en puissances impairs de $|\theta|$. En multipliant par $\operatorname{sgn}\left(\sin \theta_{G}\right)=\operatorname{sgn}(\theta)$, au moins pour $|\theta|$ petit, comme exigé par la formule (1.3.25), nous obtenons une fonction formellement lisse dans un voisinage de $\theta=0$. Par conséquent, quitte à remplacer $f_{1}$ par une fonction à petit support dans des voisinages de 0 et $\pi$, nous pouvons supposer que $f_{1}$ est lisse sauf en ces deux points, où ses singularités sont obtenues en remplaçant $\theta_{f^{G}}^{G}$ par $\theta_{e}^{G}$ dans la formule (1.3.25). Le saut de la dérivée d'ordre $k$ est alors 0 pour les dérivées d'ordre impair et, grâce à la formule (2.1.13), pour la dérivée d'ordre $2 n$ est égal à

$$
\pm i(-1)^{n} A_{n}^{ \pm}
$$

avec l'indice + en $\theta=0$ et l'indice - en $\theta=\pi$. Le coefficient $A_{n}^{ \pm}$est celui de la formule (2.1.8). Pour le saut de $2 f_{1}$ il faut multiplier par 2 . On obtient alors pour le développement asymptotique des coefficients de Fourier de $2 f_{1}$ — dans le sens de (1.3.24) — l'expression

$$
c_{m} \sim \frac{i}{\pi} \sum_{k=0}^{\infty} \frac{1}{m^{2 k+1}} A_{k}^{+}-\frac{i}{\pi} \sum_{k=0}^{\infty} \frac{(-1)^{k}}{m^{2 k+1}} A_{k}^{-} .
$$

Observons qu'il y a un facteur $i(-1)^{k}$ contribué par (1.3.26) et un facteur $i^{2 k+1}$ contribué par (1.3.24).

On démontre facilement que la limite lorsque $z \rightarrow 0$ de

$$
\ln \left(1-z e^{i \theta}\right)+\ln \left(1-z e^{-i \theta}\right) .
$$

existe et que le produit de -1 et de sa dérivée est égal à $2 \delta$. Le développement de Fourier de cette fonction est

$$
\sum_{m \neq 0} \frac{z^{|m|}}{|m|} e^{-i m \theta}
$$


Il est évident que sa limite comme distribution existe et que le développement de cette distribution est donné par

$$
\sum_{m \neq 0} \frac{1}{|m|} e^{-i m \theta}
$$

Celui de sa dérivée multipliée par -1 est

$$
d_{m}=\sum_{m \neq 0} i \operatorname{sgn}(m) e^{-i m \theta}
$$

Par conséquent,

$$
2 \pi c_{m} d_{m} \sim-2 \sum_{k=0}^{\infty} \frac{1}{|m|^{2 k+1}} A_{k}^{+}+2 \sum_{k=0}^{\infty} \frac{(-1)^{m}}{|m|^{2 k+1}} A_{k}^{-}
$$

La limite de la contribution de l'ensemble d'éléments réguliers et déployés est plus facile à calculer. L'expression (1.3.22) est multipliée par $\theta_{G}\left(a_{G}\right), \pm a\left(t_{G}\right) \mapsto a_{G}$ et intégrée sur l'image dans $\mathfrak{A}_{G}$ des éléments déployés réguliers. Toutes les difficultés viennent d'un voisinage de $t_{g}= \pm 0$. Cette fois le coefficient $c_{m}^{\prime}$ du développement de Fourier est, à une contribution rapidement décroissante près,

$$
2 \sum_{ \pm}( \pm 1)^{m-1} \int_{0}^{\infty} e^{-|m| t} \phi_{ \pm}(t) d t
$$

où $\phi_{ \pm}(t)$ a son support dans un petit voisinage $(-\epsilon, \epsilon)$ de $t=0$ et des dérivées en $t=0$ égales à celles de $\theta^{G}\left(a_{G}\right), \pm a(t) \mapsto a_{G}$.

On a

$$
\begin{aligned}
2 \int_{0}^{\epsilon} \phi_{ \pm}(t) e^{-|m| t} d t & =-\left.\frac{2}{|m|} \phi_{ \pm}(t) e^{-m t}\right|_{0} ^{\epsilon}+\frac{2}{|m|} \int_{0}^{\epsilon} e^{-|m| t} \phi_{ \pm}^{\prime}(t) d t \\
& =\frac{2}{|m|} \phi_{ \pm}(0)+\frac{2}{|m|} \int_{0}^{\epsilon} e^{-|m| t} \phi_{ \pm}^{\prime}(t) d t \\
& =\frac{2}{|m|} \phi_{ \pm}(0)+\frac{2}{|m|^{2}} \phi_{ \pm}^{\prime}(0)+\frac{2}{|m|^{2}} \int_{0}^{\epsilon} e^{-|m| t} \phi_{ \pm}^{\prime \prime}(t) d t
\end{aligned}
$$

On peut continuer. Puisque $\phi_{ \pm}$est une fonction paire, la moitié des termes s'annule. Les autres sont donnés par la formule (2.1.8). La forme asymptotique des coefficients $c_{m}^{\prime}$ est donc

$$
c_{m}^{\prime} \sim 2 \sum_{k=0}^{\infty} \frac{1}{|m|^{2 k+1}} A_{k}^{+}-2 \sum_{k=0}^{\infty} \frac{(-1)^{m}}{|m|^{2 k+1}} A_{k}^{-} .
$$

Les expressions (1.3.27) et (1.3.28) s'annulent réciproquement. 
1.4. Les tores elliptiques sur les corps $p$-adiques. Nous allons utiliser les formules de l'article [SS] pour les caractères mais dans une forme modifiée par le point de vue introduit dans ma lettre à André Weil et développé par la suite pour $G L(2)$ et $S L(2)$ dans $[\mathrm{JL}]$ et $[\mathrm{LL}]$. D'abord ce sont les caractères stables tempérés qui nous intéressent et non pas les caractères eux-mêmes. En plus, nous utilisons leur paramétrisation par les homomorphisms du groupe de Weil dans ${ }^{L} G=P G L(2, \mathbb{C})$. Quoiqu'il n'y en a guère de doute, il faut néanmoins avouer que je n'ai pas vérifié que l'identification des paramètres de [JL] avec ceux de Sally-Shalika implicite dans les calculs qui suivent est correcte, c'està-dire que les fonctions $L$ sont compatibles. Elle est simplement la seule identification raisonnable.

Les résultats de [SS] sont donnés dans trois tableaux et la lecture de cette partie de l'article exige des renvois répétés à ceux-ci. Puisque nous traitons les caractères stables, quelques-unes des complexités de leurs formules disparaissent. Sauf pour les représentations de leur troisième tableau, les $L$-paquets de la série discrète contiennent deux éléments $\pi^{+}$et $\pi^{-}$et le caractère stable est

$$
\chi_{\pi^{\text {st }}}=\chi_{\pi^{+}}+\chi_{\pi^{-}} .
$$

La différence $\chi_{\pi^{+}}-\chi_{\pi^{-}}$est traitée par le transfert endoscopique, qui n'est pas le sujet de cet article. Pour le moment, la seule autre possibilité est qu'un paquet contienne quatre éléments. C'est le cas des repésentations du troisième tableau. Dans ce cas le caractère stable est une somme

$$
\chi_{\pi^{\mathrm{st}}}=\sum_{1}^{4} \chi_{\pi_{i}} .
$$

Il serait utile lorsque nous commençons à utiliser les formules de [SS] de rappeler que l'ensemble des déterminants du centralisateur dans $G L(2)$ d'un sous groupe de Cartan $T$ de $G=S L(2)$ rattaché à une extension $E / F$ d'ordre deux est $\mathrm{N} E^{\times}$. Si le tore est déployé de sorte que $E=F \oplus F$, donc $\mathrm{N}^{\times}=F^{\times}$. Sinon il est un sous-groupe d'ordre deux de $F^{\times}$. Les déterminants du normalisateur de $T$ donnent $\pm \mathrm{N} E^{\times}$. Puisque $\left[F^{\times}: \mathrm{N} E^{\times}\right]=2$ lorsque $T$ n'est pas déployé, tout déterminant peut être réalisé dans le normalisateur si et seulement si $-1 \notin \mathrm{N} E^{\times}$. Par conséquent, si $T$ est non ramifié, donc si $E / F$ est non ramifié, il y a toujours un tore conjugué à $T$ dans $G L(2)$, mais pas dans $S L(2)$, quoique si $T$ est ramifié, il est ainsi si et seulement si -1 n'est pas un carré dans $F$. Donc, comme remarqué par Sally-Shalika, il y a soit quatre classes de conjugaison de tores elliptiques (non déployés), soit six. S'il y en a six, alors, avec la notation de [SS], $\operatorname{sgn}_{\tau}(-1)=1$ et $-\operatorname{sgn}(\tau)=-1$, de sorte que la modification $H(\Phi, V) \mapsto-\operatorname{sgn}_{\tau}(-1) H(\Phi, V)$ de $[\mathrm{SS}]$ mène, après la stabilisation, à la disparition de $H(\Phi, V)$ dans ces formules. Si, par contre, $\operatorname{sgn}_{\tau}(-1)=-1$, alors $\lambda$ et $\lambda^{-1}$, traités, en suivant [SS], comme deux éléments de $T(F)$, sont stablement conjugués mais non conjugués et le facteur

$$
\operatorname{sgn}_{\tau}\left(\frac{\lambda-\lambda^{-1}}{2 \sqrt{\tau}}\right)
$$


entraîne encore l'élimination du terme qui contient $H(\Phi, V)$. Nous employons toute la notation de $[\mathrm{SS}]$ sans répéter les définitions.

Pour le corps réel, nous avons d'abord répondu à la question A et ensuite à la question B. Nous passons par les deux mêmes étapes pour le cas des corps $p$-adiques. Soit $q=q_{v}$ le nombre d'éléments dans le corps résiduel de l'anneau $\mathcal{O}=\mathcal{O}_{v}$ des entiers de $F=F_{v}$. Nous ne l'avons pas encore écrit explicitement mais la norme choisie est telle qu'un générateur $\varpi$ de l'idéal maximal du corps de base $F$ est de norme $|\varpi|_{F}=|\varpi|=q^{-1}$. Nous définissons à partir de cette norme une norme $|\cdot|_{F}$ sur toutes les extensions finies $E$ de $F$ en exigeant que sa restriction à $F$ est la norme sur $F$ qui nous venons de fixer. Je souligne qu'avec cette définition $|x|_{E}=|x|_{F}^{[E: F]}$. Nous n'aurons pas d'occasion d'utiliser les normes $|\cdot|_{E}$. Par conséquent, nous continuons d'utiliser la notation $|x|=|x|_{F}$. Sally et Shalika utilisent la norme $|\cdot|_{F}$ en l'appelant une valuation.

Nous avons deux sortes de tore elliptique $H$ : non ramifié et ramifié. Pour le premier il faut traiter, dans la première étape, la définition du transfert $f^{G} \rightarrow f^{H}$ pour des fonctions à support dans l'ensemble d'éléments qui sont réguliers et semi-simpls et qui sont soit déployés, soit elliptiques et non ramifiés, soit elliptiques et ramifiés. Si $H$ est ramifié, il faut distinguer entre les éléments ramifiés stablement conjugués à un élément de $H(F)$ et les éléments qui ne sont pas conjugués à un tel élément. Rappelons qu'il y a, à isomorphisme près, deux tores $T$ ramifiés, parce qu'il y a deux extensions $E / F$ quadratiques et ramifiées. Il y a donc deux sortes de classes de conjugaison ramifiées, selon l'extension ramifiée engendrée par les valeurs propres d'un élément de la classe. Les tores, au moins ceux qui interviennent dans notre discussion, jouent deux rôles: celui d'un groupe $H$ qui dans le cas d'un groupe $G$ général n'est ni un tore ni un sous-groupe de $G$, mais un groupe réductif dont le groupe $L$ est pourvu d'un homomorphisme $\phi:{ }^{L} H \rightarrow{ }^{L} G$; mais en plus celui d'un sous-groupe de Cartan de $G$. Il est prudent d'utiliser la notation $T$ pour cette deuxième sorte de tore. Nous pouvons même ajouter des indices: $T_{\mathrm{sp}}$ pour un sous-groupe de Cartan déployé; $T_{\text {unr }}$ pour un sous-groupe non ramifié; et $T_{\text {ram }}$ pour un des sous-groupes ramifiés, dont il y a deux classes.

Sally-Shalika emploient deux paramètres, un caractère additif $\Phi$ de $F$ et un caractère multiplicatif dans l'ensemble des éléments de norme 1 dans l'extension quadratique rattachée à $H$ que nous supposons elliptique, car le cas où $H$ est déployé a été traité dans la section $\S 1.2$. Le caractère additif est un paramètre à l'intérieur du paquet $L$ et pour passer à un caractère stable il faut faire la somme sur $\Phi=\Phi_{b}$, donc sur $b$ modulo les carrés de $F^{\times}$. Soit $\tilde{\psi}$ une extension de $\psi$ à $E^{\times}$. La représentation $\operatorname{Ind}_{W_{E}}^{W_{F}} \tilde{\psi}$ dans $G L(2)$ donne après un passage à $P G L(2)$ l'homomorphisme rattaché dans $\S 1.1$ à $\tilde{\xi}$, où $\xi=\psi$. Cette représentation est, à mon avis, le paramètre maintenant généralement employé pour le $L$-paquet des représentations rattachées dans [SS] au paramètre $\psi$. On vérifie que les caractères $\psi$ et $\psi^{-1}$ donnent les mêmes paramètres et les mêmes paquets et que les paramètres et paquets définis de cette façon sont différents sauf si $\psi$ est d'ordre 2. Ceci est le cas exceptionnel de la section $\S 1$. Les trois extensions quadratiques $E_{1}, E_{2}, E_{3}$ et les caractères d'ordre deux des groupes $\left\{x \in E_{i}^{\times} \mid \mathrm{N}_{E_{i} / F} x=1\right\}$ rattachés aux extensions quadratiques $K / E_{i},[K: F]=4, E_{i} \subset K$ pour tout $i$, donnent des représentations qui appartiennent au même paquet. Les conséquences sont expliquées dans [LL]. Ces représentations sont 
décrites dans le troisième tableau. Si $\psi$ est trivial, la représentation y rattachée est dans la série principale.

\section{Question A: Cas où $H$ est non ramifié}

Examinons la question A, d'abord pour un tore $H$ non ramifié et ensuite pour un tore ramifié. Le deuxième tableau de [SS] nous donne les analogues des formules (1.3.1), sauf que les caractères dans le tableau ne sont pas stabilisés. Il s'avère qu'une stabilisation n'est pas absolument nécessaire au début mais nous préférons qu'elle soit introduite tout de suite. Nous traitons d'abord une fonction $f^{G}$ dont le support est contenu dans l'ensemble des éléments réguliers, semi-simples et déployés. Dans [SS] les deux valeurs propres $\gamma_{1}, \gamma_{2}$ d'un élément $\gamma_{G}$ sont dénotées $\lambda, \lambda^{-1}$ et nous utilisons leur notation dans les renvois aux tableaux. Observons aussi que le conducteur de [SS] n'est pas le conducteur habituel, car leurs caractères $\psi$ ne sont pas des caractères de $E^{\times}$mais de l'ensemble des éléments dans $E^{\times}$de norme 1 .

La formule (1.3.2) reste valable, sauf qu'il faut distinguer maintenant entre le tore $H$ et des sous-groupes de Cartan $T$ de $G$. Comme nous l'avons observé, la possibilité qu'un des $T$ soit isomorphe à $H$ est plus ou moins une particularité du cas simple que nous traitons. Cette formule devient non plus une somme sur $m$ mais une somme sur l'ensemble de caractères de $H(F)$,

$$
\Theta\left(\gamma_{H}, t_{G}\right) d a_{H}=\sum_{\psi} \chi_{\pi_{G}^{\mathrm{st}}}\left(t_{G}\right) \bar{\psi}\left(\gamma_{H}\right) d a_{H}
$$

où $\pi_{H}^{\mathrm{st}}=\pi_{H}^{\mathrm{st}}(\psi)=\psi$ et $\pi_{G}^{\mathrm{st}}$ est l'image par fonctorialité de $\pi_{H}^{\mathrm{st}}$. Puisque, d'une part, nous voulons souligner que les formules données ici sont censées être des cas spéciaux d'une théorie générale et, de l'autre part, nos formules pour le groupe abélien $H$ sont des expressions de la théorie abélienne, nous préférons utiliser non seulement une notation qui soit convenable pour cette théorie simple mais aussi une notation convenable pour les transferts qui apparaissent dans la fonctorialité. La solution la plus simple est de nous permettre deux notations pour le même objet. Observons aussi que pour $H$ la base de Steinberg-Hitchin est multiplicative.

La formule (1.2.5) est une conséquence de la formule (3.31) de [FLN]. Une autre façon de l'écrire est

$$
\int\left(\sum_{a_{H} \mapsto a_{G}} \frac{h\left(a_{H}\right)}{\left|\Delta_{G}\left(a_{G}\right)\right|}\right) d a_{G}=\int \frac{h\left(a_{H}\right)}{L\left(1, \sigma_{H}\right)} d a_{H} .
$$

Dans cette formule $H$ est n'importe quel sous-groupe de Cartan de $G$ et $a_{H}=\gamma_{H}$. La fonction $h$ est essentiellement arbitraire. La formule (1.3.4) est une façon d'écrire le côté droit de (1.1.7). En général, une formule semblable décrit le côté droit de la formule dans le cas d'une fonction $f^{G}$ dont le support est contenu dans l'ensemble d'éléments réguliers stablement conjugués à un élément d'un sous-groupe de Cartan $T$ donné. Avant d'y substituer la formule (1.4.3), nous l'écrivons

$$
\int\left\{\lambda\left(a_{G}\right)\left|\Delta\left(a_{G}\right)\right| \operatorname{Orb}^{\mathrm{st}}\left(a_{G}, f^{G}\right)\right\}\left\{\left|\Delta\left(a_{G}\right)\right| \Theta\left(a_{H}, a_{G}\right)\right\} d_{\text {geom }} \gamma_{G}, \quad \gamma_{G} \mapsto a_{G}
$$


Il est très difficile à ne pas se perdre dans la notation et dans les diverses mesures. Il y a la mesure géométrique $d_{\text {geom }} \gamma_{G}$ sur les tores $T$ qui intervient car $\left|\Delta\left(a_{G}\right)\right| d_{\text {geom }} \gamma_{G}=d a_{G}$. Par la suite nous écrirons l'intégrale (1.4.4) comme une somme sur un ensemble de représentants $T$ des classes de conjugaison stable des sous-groupes de Cartan. Pour chacun de ces représentants $T$, l'intégration sera faite sur un ensemble de représentants dans $T(F)$ des classes de conjugaison stables et régulières qui ont une intersection non vide avec $T(F)$.

Il est bien connu et, en plus, cela découle de la section $\S 2.2$, que le premier facteur est une fonction majorée par une constante. Le premier facteur dans l'intégrale (1.4.4) est $\theta_{f^{G}}^{G}\left(a_{G}\right)$; le deuxième facteur est

$$
\sum_{\psi}\left|\Delta\left(a_{G}\right)\right| \chi_{\pi_{G}^{\mathrm{st}}}\left(\gamma_{G}\right) \bar{\psi}\left(\gamma_{H}\right)
$$

Il est aussi connu mais, en particulier, cela découle des formules de [SS], que pour un $\psi$ donné le coefficient

$$
\left|\Delta\left(a_{G}\right)\right| \chi_{\pi_{G}^{\mathrm{st}}}\left(\gamma_{G}\right), \quad \gamma_{G} \mapsto a_{G},
$$

est une fonction bornée de $\gamma_{G}=t_{G}$. Sous les hypothèses en force en ce moment, son support dans $T(F)$, et dans l'image de $T(F)$ dans $\mathfrak{A}_{F}$, est compact. Il en résulte que, pour n'importe quel $T$, la contribution d'un seul terme à (1.4.4), donc du terme rattaché à un $\psi$ donné, est le produit d'une constante avec $\bar{\psi}$, ce qui est certainement une fonction lisse. Nous pouvons donc le mettre au rancart.

Le caractère trivial, donc le caractère de conducteur $h=0$, est exclu des tableaux de [SS] car il donne une représentation de la série principale. Il est préférable, par conséquent, de le traiter séparément au début et de l'exclure des calculs subséquents, donc de l'analogue de la somme (1.3.20), ce que nous pouvons, selon le paragraphe précédent, faire. Le terme exceptional dans (1.3.20) aurait été celui pour lequel $m=0$, le caractère sur le tore déployé étant $t \mapsto \operatorname{sgn}(t)$. Maintenant le caractère de $H$ est trivial, mais le caractère sgn sur $\mathbb{R}$, donc le caractère quadratique rattaché à l'extension $\mathbb{C} / \mathbb{R}$, est remplacé par le caractère quadratique $\xi_{E / F}$ rattaché à l'extension $E / F$.

Passons maintenant aux calculs pour lesquels nous avons besoin des tableaux. Nous commençons avec le tore $H$ non ramifié. Nous supposons que le support de $f^{G}$ ne contient pas $\pm I$. Il y a donc d'une part un entier $k \geq 0$, tel que $f^{G}(g)=0$ si les valeurs propres $\lambda^{ \pm 1}$ sont telles que $| \pm 1-\lambda|>q^{-k}$. De l'autre part, il résulte des formules du deuxième tableau que pour $T$ déployé ou ramifié les caractères rattachés à un caractère donné $\psi$ avec un conducteur $h$ donné sont 0 là où $| \pm 1-\lambda|>q^{-h}$. Donc pour un $f^{G}$ donné à support contenu dans l'ensemble des classes de conjugaison stables qui contient un élément régulier et semi-simple de $\mathrm{T}(\mathrm{F})$, la contribution de $\psi$ à (1.4.5) est 0 si $h \geq k$. Par conséquent, il n'y a qu'un nombre fini de $\psi$ qui contribuent à cette somme et la réponse à la question $\mathrm{A}$ est affirmative.

Pour le cas où $T$ est non ramifié, donc isomorphe à $H$, la réponse est encore affirmative, mais la démonstration n'est pas si évidente. Nous l'avons déjà vu pour le corps des nombres réels lorsque $H$ et $T$ étaient elliptiques. Nous aurons besoin des quatre dernières lignes 
du deuxième tableau dans lesquelles $\lambda=\alpha+\sqrt{\epsilon} \beta$. Observons que les matrices qui $\mathrm{y}$ interviennent,

$$
\left(\begin{array}{cc}
\alpha & \beta \\
\epsilon \beta & \alpha
\end{array}\right), \quad\left(\begin{array}{cc}
\alpha & \beta \tau \\
\epsilon \beta \tau^{-1} & \alpha
\end{array}\right)
$$

sont stablement conjuguées mais non conjuguées, de sorte qu'il faut ajouter leurs deux contributions. Le résultat est que les termes qui contiennent le facteur $H(\Phi, V)$ s'enlèvent mutuellement et qu'un facteur 2 y est introduit. Le coefficient $\alpha_{\psi}$ de $\bar{\psi}\left(\gamma_{H}\right)$ dans le développement de $\left|\Delta\left(a_{G}\right)\right| \Theta\left(a_{H}, a_{G}\right)$ est

$$
-2 q^{h-1}\left|\lambda-\lambda^{-1}\right|
$$

si $|1-\lambda| \leq q^{-h}$, et

$$
(-1)^{h} \operatorname{sgn}_{\epsilon}\left(\frac{\lambda-\lambda^{-1}}{2 \sqrt{\epsilon}}\right)\left(\psi(\lambda)+\psi\left(\lambda^{-1}\right)\right)
$$

si $q^{-h}<|1-\lambda| \leq 1,|1+\lambda|=1$. En principe, nous devons aussi examiner le comportement près de $\lambda=-1$, mais puisque les formules pour le caractère en $-t_{G}$ se déduisent facilement de celles pour le caractère en $t_{G}$, nous pouvons, comme Sally et Shalika le remarquent, nous restreindre aux domaines (1.4.7) et (1.4.8).

Dans un ensemble dans lequel $|1-\lambda|$ est minoré par un nombre positif, il n'y qu'un nombre fini de coefficients (1.4.7) qui ne sont pas égaux à 0. Leur contribution à l'intégrale (1.4.4) sera une fonction lisse de $a_{H}=\gamma_{H}$. Par conséquent, sous nos hypothèses il suffit de considérer non pas l'intégrale (1.4.4) mais l'intégrale d'un produit d'une fonction $\theta=$ $\theta^{G}=\theta_{f^{G}}^{G}$ de la variable $a_{G}$, à présent lisse et à support compact dans l'image de $T^{\mathrm{reg}}(F)$, avec la distribution

$$
\sum_{\psi}(-1)^{h} \operatorname{sgn}_{\epsilon}\left(\frac{\lambda-\lambda^{-1}}{2 \sqrt{\epsilon}}\right)\left(\psi\left(\lambda \mu^{-1}\right)+\psi\left(\lambda^{-1} \mu^{-1}\right)\right),
$$

où $\mu$ est $\gamma_{H}$, mais traité comme un élément de norme 1 dans $E^{\times}$. Il s'agit au fond d'un produit de convolution ou, plus précisément, de la somme de deux tels produits. Si nous multiplions $\theta$ avec le facteur

$$
\operatorname{sgn}_{\epsilon}\left(\frac{\lambda-\lambda^{-1}}{2 \sqrt{\epsilon}}\right)
$$

nous obtenons une fonction $\theta^{\prime}$ avec les mêmes propriétés, car les singularités de (1.4.10) sont aux points $a_{G}= \pm 2$. L'intégrale du produit de $\theta^{\prime}$ et $\psi\left(\lambda^{ \pm 1}\right)$ est zéro pour presque tout $\psi$. Il en résulte que le produit de convolution est une somme finie de caractères et par conséquent lisse. La réponse à la question $\mathrm{A}$ est donc affirmative si le tore $H$ est non ramifié.

Question A: Cas où $H$ est ramifié 
Elle est aussi affirmative pour un tore $H$ ramifié et la démonstration est presque la même. Le premier tableau est le tableau pertinent. Pour le tore $T$ déployé ou non ramifié, l'argument ne diffère guère de celui pour $H$ non ramifié et $T$ déployé ou ramifié. C'est aussi le cas pour $H$ ramifié et $T$ ramifié mais non isomorphe, sauf que le facteur (1.4.10) et, par conséquent, la fonction $\theta^{\prime}$ sont légèrement plus compliqués. Les lignes du tableau pertinentes sont celles pour lesquelles $\lambda=\alpha+\sqrt{\epsilon \tau} \beta$. Grâce à notre hypothèse sur la fonction $f^{G}, \theta\left(a_{G}\right), t \mapsto a_{G}$ est à support dans une région $| \pm 1-\lambda| \geq \delta>0$. Quoique la formule pour le caractère dans ce cas, au moins pour $|1-\lambda|=q^{-h+1 / 2}$, est plus compliquée, la conclusion reste la même. La fonction $f^{H}$ définie par le produit de convolution est la somme d'un nombre fini de caractères et, par conséquent, est lisse.

Supposons enfin que $H$ et $T$ soient ramifiés et isomorphes. C'est le cas $\lambda=\alpha+\sqrt{\tau} \beta$ du tableau. Les formules deviennent plus simples. Pour des raisons déjà données, le facteur $H(\Phi, V)$ s'enlève partout et ce qui reste est multiplié par 2. Encore une fois, ce sont les coefficients des $\psi$ pour lesquels $q^{-h+1 / 2}<|1-\lambda|<1$ qui s'avèrent pertinents. Le coefficient de $\psi$ dans le produit de convolution contient le facteur $(-1)^{a} S(\psi), a=a(\psi) \in \mathbb{Z}$, qui peut être introduit après avoir calculé les intégrales. Ce qui reste est tout à fait semblable à (1.4.9). Le caractère $\operatorname{sgn}_{\epsilon}$ est remplacé $\operatorname{par} \operatorname{sgn}_{\tau}$ et le facteur $(-1)^{h} \operatorname{par}(-1)^{a} S(\psi)$.

\section{Question B: Cas où $H$ est non ramifié}

La réponse à la question $\mathrm{B}$ est encore positive et sa démonstration semblable à celle pour le corps de nombres réels. Elle est donc plus fastidieuse que ce que nous venons de faire et exige un recours, assez facile et qu'il n'est guère nécessaire d'expliciter, aux approximations de (1.3.23). Dans l'article FLN on a remarqué que la proposition 5.6 était dans le cas du groupe $S L(2)$ susceptible d'une démonstration élémentaire, mais nous ne l'avons pas donnée, préférant donner une démonstration géométrique générale. Il n'y guère de doute qu'il y a des constructions générales des flèches $f^{G} \mapsto f^{H}$, donc des constructions qui répondent aux questions $\mathrm{A}$ et $\mathrm{B}$. La similarité entre les calculs élémentaires présentés ici et ceux dans la démonstration élémentaire de la proposition 5.6 de [FLN] suggère que ces constructions seront géométriques, donc liées à la démonstration géométrique de cette proposition. Je suppose qu'un début serait une démonstration géométrique des résultats de $[S]$. Dans cet article je me contente d'une démonstration plus élémentaire.

Nous avons constaté que tant que le support de la fonction $\theta^{G}=\theta_{f}^{G}$ est contenu dans l'ensemble $\left|a_{G} \mp 2\right|>\delta, \delta>0$, mais autrement arbitraire, donc tant que les intégrales orbitales $\operatorname{Orb}^{\mathrm{st}}\left(t_{T}, f^{G}\right)$ sont 0 pour $| \pm 1-\lambda|>\delta$, pour n'importe quel $\delta$ positif, les fonctions $f^{H}$ rattachées à $f^{G}$ sont lisses. Nous pouvons par conséquent remplacer $f^{G}$ par une fonction dont les intégrales orbitales stables sont 0 dans cette région mais égales à celles de $f^{G}$ lorsque $| \pm 1-\lambda| \leq \delta$. Le nombre positif $\delta$ doit être assez petit pour que les formules (2.2.1) soient valables pour $| \pm 1-\lambda| \leq \delta$. Il suffit de démontrer que pour cette nouvelle fonction $f^{G}$ la distribution en $a_{H}$ donnée par (1.4.4) est une fonction lisse. Cette distribution ne dépend que des quatres nombres $\alpha_{ \pm}, \beta_{ \pm}$et nous pouvons supposer que tous sauf un sont zéro et que l'un est égal à 1 . Il suffit de traiter le cas où ceci est $\beta_{+}$ou $\alpha_{+}$.

L'intégrale (1.4.4) est la somme des intégrales sur les images des diverses classes de sousgroupes de Cartan, donc sous l'hypothèse que $q$ est impair, sur quatre sous-ensembles, à 
savoir sur les images des tores $T_{\mathrm{sp}}, T_{\mathrm{unr}}$ et des deux tores ramifiés $T_{\mathrm{ram}}^{\prime}, T_{\mathrm{ram}}^{\prime \prime}$. Le deuxième facteur est une somme sur les caractères de $H$ avec des coefficients donnés par (1.4.6). Les coefficients du développement de la distribution sur $H$ rattachée à $f^{G}$ sont donnés par l'intégration du produit de $\theta=\theta_{f^{G}}^{G}$ et (1.4.6). Pour démontrer que cette distribution est donnée par une fonction lisse, il suffit de montrer que tous sauf un nombre fini de ces coefficients sont 0 . Supposons que $\delta=q^{-m}$ avec un entier positif $m$. Puisqu'il n'y qu'un nombre fini de caractères de conducteur plus petit ou égal à $m$, il suffit de traiter les $\psi$ de conducteur $h$ plus grand que $m$, donc au moins 2, car il n'y a qu'un nombre fini de caractères avec un conducteur donné. Observons que cette hypothèse exclut les caractères du troisième tableau de [SS].

Nous voulons donc montrer que si nous utilisons (1.4.3) pour développer (1.4.4) en puissances de $\bar{\psi}\left(\gamma_{H}\right)$, alors le coefficient est 0 si le conducteur de $\psi$ est plus grand ou égal à $m$ lorsque

(i) $\alpha_{-}=\beta_{-}=0$ et $\beta_{+}=1, \alpha_{+}=0$ ou $\beta_{+}=0, \alpha_{+}=1$,

(ii) $H$ est le tore non ramifié ou un des deux tores ramifiés.

Nous traitons d'abord le cas d'un tore non ramifié. Nous nous permettons de supposer dans les calculs que la mesure locale est définie par rapport à un caractère local pour lequel l'ensemble $\mathcal{O}_{v} \subset F_{v}$ est auto-dual, de sorte que sa mesure est 1. Autrement, il faudrait traîner dans tous les calculs une constante inutile.

Commençons avec $\alpha_{+}=0, \beta_{+}=1$ et calculons les contributions de $T_{\mathrm{sp}}, T_{\mathrm{unr}}$, et celles de $T_{\text {ram }}^{\prime}$ et $T_{\text {ram }}^{\prime \prime}$, ces dernières étant égales, à partir des formules du deuxième tableau. Il y aura des facteurs 2 pour tenir compte de la stabilisation ou de la présence de deux sous-groupes de Cartan ramifiés.

(i) Pour le tore $T_{\mathrm{sp}}$ la contribution est

$$
2\left\{\int_{|1-\lambda| \leq q^{-h}} \frac{d_{\text {geom }} \lambda}{|\lambda|}-q^{h-1} \int_{|1-\lambda| \leq q^{-h}}\left|1-\lambda^{2}\right| \frac{d_{\text {geom }} \lambda}{|\lambda|}\right\} .
$$

Lorsque $\lambda$ est proche de 1 , nous avons $|\lambda|=1$ de sorte que

$$
\frac{d_{\text {geom }} \lambda}{|\lambda|}=d_{\text {geom }} \lambda=d \lambda
$$

Puisque nous supposons que $q$ est impair, nous avons $\left|1-\lambda^{2}\right|=|1-\lambda|$ dans la région d'intégration. Cette contribution est, par conséquent, donnée par

$$
2\left(1-\frac{1}{q}\right)\left\{\frac{q^{-h}}{1-1 / q}-\frac{q^{-h-1}}{1-1 / q^{2}}\right\}=q^{-h}\left\{2-\frac{2}{q(1+1 / q)}\right\}=q^{-h} D_{\mathrm{sp}}^{\mathrm{unr}} .
$$

La dernière égalité sert à définir $D_{\mathrm{sp}}^{\mathrm{unr}}$.

(ii) Pour le tore $T_{\text {unr }}$ ce sont les lignes $\lambda=\alpha+\sqrt{\epsilon} \beta$ du deuxième tableau qui sont pertinentes. Une fois que les termes dans lesquels le facteur $H(\Phi, V)$ sont enlevés par la stabilisation, ce qui reste contient le caractère $\operatorname{sgn}_{\epsilon}$ dont l'influence n'est pas évidente. Un 
élément $\mathrm{du}$ tore, ou plutôt sa valeur propre $\lambda$, s'écrit comme $\lambda=\alpha+\sqrt{\epsilon} \beta$ ou comme $a_{0}+a_{1} \varpi+a_{2} \varpi^{2}$ où $\varpi=\varpi_{F}=\varpi_{E}$ et òu $a_{1}, a_{1}, \ldots$ sont des représentants de $\mathcal{O}_{E}$ modulo $\mathfrak{p}_{E}$. On a $a_{0} \bar{a}_{0} \equiv 1\left(\bmod \mathfrak{p}_{E}\right)$ et $a_{0} \equiv \pm 1\left(\bmod \mathfrak{p}_{E}\right)$. Observons que

$$
\operatorname{sgn}_{\epsilon}\left(\frac{\lambda-\lambda^{-1}}{2 \sqrt{\epsilon}}\right)=\operatorname{sgn}_{\epsilon}(\beta)
$$

De la formule (1.4.9) et de notre hypothèse sur la fonction $\theta_{f G}^{G}$, il résulte que le coefficient de $\psi$ dans le développement de la distribution rattachée à $f^{G}$ est donné par une somme de deux intégrales, dont la première est l'intégrale de

$$
(-1)^{h} \operatorname{sgn}_{\epsilon}(\beta)\left\{\psi(\lambda)+\psi\left(\lambda^{-1}\right)\right\}
$$

sur l'ensemble $q^{-h}<|1-\lambda| \leq q^{-m}$. L'entier $m$ est donné par $f^{G}$ et $h$ par $\psi$. La deuxième vient des lignes pour $0 \leq|1-\lambda| \leq q^{-h}$. Elle est, à un facteur 2 près,

$$
-q^{h-1} \int_{|1-\lambda| \leq q^{-h}}\left|\lambda-\lambda^{-1}\right| d \lambda=-q^{h-1}(1-1 / q) \sum_{k=h}^{\infty} q^{-2 k}=-\frac{q^{-h-1}}{1+1 / q} .
$$

Le facteur 2 provient de la stabilisation.

Pour calculer la contribution de (1.4.11) nous considérons les intégrales sur les couronnes $|1-\lambda|=q^{-k}, h>k \geq m$ à tour de rôle. Si $|1-\lambda|=q^{-k}$ alors $\lambda=1+a_{k} \varpi^{k}+\ldots$ et $\bar{a}_{k} \equiv-a_{k}\left(\bmod \mathfrak{p}_{E}\right)$. Nous prenons donc $a_{k}=b_{k} \sqrt{\epsilon}, b_{k} \in \mathcal{O}_{F},\left|b_{k}\right|=1$. Évidemment $\operatorname{sgn}_{\epsilon}(\beta)=(-1)^{k}$ de sorte qu'il est constant sur chaque couronne. Si $k+1 \leq h$ le caractère $\psi$ n'est pas trivial sur le groupe $\left\{\mu|| \mu-1 \mid \leq q^{k-1}\right\}$ mais la couronne $|1-\lambda|=q^{-k}$ est invariant par rapport à ce groupe. Il en résulte que l'intégrale sur la couronne est égale à 0 .

Il ne reste que la couronne $k+1=h$. Sur cette couronne le produit de $(-1)^{h}$ et $\operatorname{sgn}_{\epsilon}(\beta)$ est égal à 1. Mais le produit mis pour le moment à côté nous pouvons ajouter l'intégrale sur la couronne de rayon $q^{-k}$ à l'intégrale sur les couronnes de rayon plus petit pour obtenir un intégrale sur le disque de rayon $q^{-k}$. Celle-ci est égale à 0 . L'intégrale sur la couronne de rayon $q^{-k}$ est donc égale à l'intégrale sur le disque de rayon $q^{-h}$ multipliée par -1 . Les deux facteurs -1 s'annulent mutuellement. L'intégrale sur le disque de rayon $q^{-h}$ est $q^{-h}$ ou plutôt $2 q^{-h}$ si on tient compte des deux termes de (1.4.11). La contribution totale du tore non ramifié $T$ est donc

$$
q^{-h}\left\{2-\frac{2}{q(1+1 / q)}\right\}=q^{-h} D_{\mathrm{unr}}^{\mathrm{unr}}
$$

Observons que $D_{\mathrm{unr}}^{\mathrm{unr}}=D_{\mathrm{unr}}^{\mathrm{sp}}$

(iii) Si $T$ est un des deux groupes de Cartan ramifiés, alors les lignes pertinentes du deuxième tableau sont celles pour lesquelles $\lambda=\alpha+\sqrt{\epsilon} \beta$ ou $\lambda=\alpha+\sqrt{\epsilon \tau} \beta$. Elles sont bien plus simples que celles pour $T$ non ramifié. La valeur absolue $|1-\lambda|$ est une puissance entière de $q^{1 / 2}$. Les lignes pertinentes du deuxième tableau sont celles pour lesquelles 
$\lambda=\alpha+\sqrt{\tau} \beta$ ou $\lambda=\alpha+\sqrt{\epsilon \tau} \beta$. Les formules sont plus simples que celles pour $T=T_{\mathrm{unr}}$. On n'a que l'analogue de la somme (1.2.12), mais cette fois elle va intervenir avec le facteur 4, le produit d'un facteur 2 introduit par la stabilisation et un facteur 2 à cause des deux tores ramifiées. La condition satisfaite par $\lambda$ est $|1-\lambda| \leq q^{-h+1 / 2}$, donc $|1-\lambda|=q^{-k-1 / 2}$, $k \geq h-1$. Nous allons vérifier que

$$
\int_{|1-\lambda|=q^{-k-1 / 2}} d \lambda=\left(1-\frac{1}{q}\right) q^{-1 / 2-k}
$$

Il en résultera que

$$
-q^{h-1} \sum_{k=h-1}^{\infty} \int_{|1-\lambda|=q^{-k-1 / 2}} q^{-1 / 2-k} d \lambda=-q^{h-1}\left(1-\frac{1}{q}\right) \sum_{k=h-1}^{\infty} q^{-1-2 k}=-\frac{q^{-h}}{1+1 / q}
$$

et que la contribution des deux tores ramifiés est $q^{-h} D_{\text {ram }}^{\text {unr }}$ où $D_{\text {ram }}^{\text {unr }}=-4 /(1+1 / q)$. Puisque

$$
D_{\mathrm{sp}}^{\mathrm{unr}}+D_{\mathrm{unr}}^{\mathrm{unr}}+D_{\mathrm{ram}}^{\mathrm{unr}}=2\left(2-\frac{2}{q(1+1 / q)}\right)-\frac{4}{1+1 / q}=0
$$

tous les coefficients de l'expansion (1.4.4) sauf un nombre fini sont 0 sous notre hypothèse sur le comportement de $\theta_{f}$.

Sans doute une méthode plus élégante de vérifier la formule 1.4 .13 existe, mais nous utilisons la proposition 3.29 de [FLN]. Pour le tore ramifié et $k \geq 0$, les conditions $|1-\lambda| \leq$ $q^{-k-1 / 2}, \lambda \bar{\lambda}=1$, équivalent à $|\lambda+\bar{\lambda}-2| \leq q^{-2 k-1}, \lambda \bar{\lambda}=1$, car

$$
(1-\lambda)(1-\bar{\lambda})=2-\lambda-\bar{\lambda}
$$

Sous nos hypothèses on a $|\Delta(t)|=|1-\lambda|=q^{-k-1 / 2}$, de sorte que l'intégrale de (1.4.13) est

$$
q^{k+1 / 2} \int_{|x|=q^{-2 k-1}} q^{-1 / 2-k} d x=q^{-2 k-1}\left(1-\frac{1}{q}\right) .
$$

Nous continuons à supposer que le tore $H$ est non ramifié mais nous supposons maintenant que $\alpha_{+}=1, \beta_{+}=0$. Le tore $T_{\mathrm{sp}}$ n'intervient pas dans les calculs. La somme de (1.4.12) est modifiée légèrement à cause du facteur supplémentaire $\Delta(t)$ dans la formule $(2.2 .1)$.

(i) Commençons avec le tore $T_{\text {unr }}$ non ramifié. Le calcul est semblable à celui déjà fait car les fonctions $\Delta(\gamma)$ sont constantes sur les couronnes. La contribution des lignes pour lesquelles $0<|1-\lambda| \leq q^{-h}$ est maintenant

$$
-2 q^{h-1}(1-1 / q) \sum_{k=h}^{\infty} q^{-3 k}=-\frac{2 q^{-2 h-1}}{1+1 / q+1 / q^{2}} .
$$

Pour $q^{-h}<|1-\lambda| \leq 1$ les calculs pour les couronnes déjà effectués montrent que seulement la couronne $k=h+1$ contribue. Cette contribution est égale au produit de celle déjà 
calculée avec $q^{-h-1}$. Ceci donne $2 q^{-2 h+1}$. Selon les formules $(2.2 .3)$, il faut multiplier la contribution du tore non ramifié avec $2 / q$. La contribution totale du ce tore est alors

$$
4 q^{-2 h}\left(1-\frac{q^{-2}}{1+1 / q+1 / q^{2}}\right)=q^{-2 h} C_{\mathrm{unr}}^{\mathrm{unr}}
$$

(ii) Les contribution des tores ramifiés sont plus simples à calculer. Il y a un facteur supplémentaire $q^{-1 / 2-k}$ dans l'équation (1.4.13) et dans les équations qui suivent. Si nous ajoutons le facteur 4 exigé par la stabilisation et la présence de deux tores ramifiés, cela donne

$$
-4 q^{h-1}\left(1-\frac{1}{q}\right) \sum_{k=h-1}^{\infty} q^{-3 / 2-3 k}=-\frac{4 q^{-2 h+1 / 2}}{1+1 / q+1 / q^{2}} .
$$

Cette fois les formules $(2.2 .3)$ exigent que cette expression soit multipliée avec $q^{-1 / 2}(1+$ $1 / q)$. Le résultat est

$$
-q^{-2 h} \frac{4(1+1 / q)}{1+1 / q+1 / q^{2}}=q^{-2 h} C_{\mathrm{ram}}^{\mathrm{unr}} .
$$

La somme vaut alors $C_{\mathrm{unr}}^{\mathrm{unr}}+C_{\mathrm{ram}}^{\mathrm{unr}}=0$.

La conclusion est que le transfert $f^{H}$ existe pour le tore non ramifié et qu'il est une fonction lisse. La réponse à la question $\mathrm{B}$ est affirmative aussi pour les tores ramifiés quoique les calculs sont même plus fastidieux.

\section{Question B: Cas où $H$ est ramifié}

Nous supposons encore que les intégrales orbitales de $f^{G}$ sont 0 sauf si $| \pm 1-\lambda| \leq \delta$, mais que dans cette région les formules (2.2.1) sont valables. Il suffit, comme pour le tore ramifié, de ne traiter la fonction $f^{H}$ que dans le voisinage de $a_{H}=\gamma_{H}=1$, donc de supposer que $\alpha_{-}=\beta_{-}=0$. Nous aurons maintenant quatre possibilités différentes à examiner, ceux où $T=T_{\mathrm{sp}}, T_{\mathrm{unr}}$, et ceux où $T=T_{\text {ram }}$ est ramifié, mais pour ceux-ci il faut distinguer le cas $T=H$ du cas $T \neq H$.

Puisqu'il s'agit de questions linéaires, il suffit de supposer soit que $\alpha_{+}=1, \beta_{+}=0$ soit que $\alpha_{+}=0, \beta_{+}=1$. Pour la première des deux possibilités, le tore $T_{\mathrm{sp}}$ n'intervient pas. Nous pouvons prendre $\delta=q^{-m}$ avec un $m=m_{\mathrm{sp}}, m_{\mathrm{unr}}$ ou $m_{\mathrm{ram}}$ qui dépend de la classe du tore $T$, mais en fait nous prenons tous ces $m$ égaux et positifs. Chaque classe de sous-groupes de Cartan contribue aux coefficients de Fourier de la distribution $f^{H}$ et il faut montrer que la somme de ces contributions est 0 pour presque tout caractère $\psi$ de $H$. Soit encore $h$ le conducteur dans le sens de [SS]. Nous supposons que $h>m$, ce qui n'exclut qu'un nombre fini de caractères.

Les formules pertinentes sont celles du premier tableau. Nous avons déjà expliqué que grâce à la formule pour $H(\Phi, V)$ qui se trouve entre le deuxième et le troisième tableau la stabilisation enlève les termes qui contiennent $H(\Phi, V)$ et multiplie les autres par un facteur 2. Commençons avec le cas $\alpha_{+}=0, \beta_{+}=1$.

(i) Pour le tore $T_{\mathrm{sp}}$ la contribution est donnée par la ligne $\lambda \in k^{\times}$du deuxième tableau. Elle est égale à

$$
2 \int_{|1-\lambda| \leq q^{-h}}\left\{1-\frac{1}{2} q^{h}\left(1+\frac{1}{q}\right)\left|\lambda-\lambda^{-1}\right|\right\} d \lambda
$$


$\mathrm{Ou}$

$$
2 \sum_{k=h}^{\infty}(1-1 / q) q^{k}-q^{h}(1+1 / q) \sum_{k=h}^{\infty}(1-1 / q) q^{-2 k}=q^{-h}
$$

(ii) La contribution des tores non ramifiés $T_{\text {unr }}$ est donnée par les deux dernières lignes $\mathrm{du}$ tableau, celles dans lesquelles $\lambda=\alpha+\sqrt{\epsilon} \beta$. Puisque $m>0$, nous avons $|\alpha-1|<1$ et

$$
\frac{d_{\mathrm{geom}} \lambda}{\lambda}=d_{\mathrm{geom}} \beta
$$

Plus précisément, $d \lambda=d \alpha+\sqrt{\epsilon} d \beta$ et l'équation $\alpha^{2}-\beta^{2} \epsilon=1$ donnent

$$
\frac{d \lambda}{\lambda}=\frac{\sqrt{\epsilon}}{\alpha} d \beta
$$

et $|\sqrt{\epsilon}|=|\alpha|=|\lambda|=1$. Nous obtenons donc

$$
-q^{h}(1+1 / q)(1-1 / q) \sum_{k=h}^{\infty} q^{-2 k}=-q^{-h}
$$

Il résulte de ces deux calculs que la somme des contributions des tores déployés et non ramifiés donne 0 . Nous montrons que la somme des contributions des deux classes de tores ramifiés est aussi 0 . Le calcul se fait par couronne comme pour le tore non ramifié mais il est plus compliqué.

(iii) La contribution des deux classes de tores ramifiés, pour lesquels $\lambda=\alpha+\sqrt{\tau} \beta$ si $T_{\text {ram }}$ est isomorphe à $H$ ou $\lambda=\alpha+\sqrt{\epsilon \tau} \beta$ sinon, se sépare dans une partie facile, celle donnée par la région $0<|1-\lambda|^{2} \leq q^{-2 h-1}$, et une partie plus difficile, celle donnée par $|1-\lambda|^{2} \geq q^{-2 h+1}$. C'est le calcul pour la deuxième partie qui se fait par couronnes. Commençons avec la partie facile.

Pour $\lambda=\alpha+\sqrt{\tau^{\prime}} \beta$, où $\tau^{\prime}=\tau$ ou $\tau^{\prime}=\epsilon \tau$, nous avons $|1-\lambda|^{2} \leq q^{-2 h-1},|\alpha-1|<1$, $|\beta|=q^{-k}, k \geq h$. En plus $\left|\lambda-\lambda^{-1}\right|=q^{-k-1 / 2}, d \lambda=q^{-1 / 2} d \beta$. Plus précisément, $d_{\text {geom }} \lambda=$ $q^{-1 / 2} d_{\text {geom }} \beta$, mais je me permets ici une notation plus informelle. La contribution de la région $0<|1-\lambda|^{2} \leq q^{-2 h-1}$ est, pour chacun des deux tores ramifiés,

$$
-q^{h}(1+1 / q) \sum_{k=h}^{\infty}(1-1 / q) q^{-2 k-1}=-q^{-h-1} .
$$

La somme des deux contributions est $-2 q^{-h-1}$. Observons que la stabilisation enlève le facteur 2 des dénominateurs de [SS] et que le facteur 2 est introduit alors à cause des deux classes stables de tores.

Les contributions pour $|1-\lambda|^{2} \geq q^{-2 h+1}$ contiennent dans leurs définitions des sommes sur $\gamma$ semblables aux sommes gaussiennes et le facteur $S(\psi)$, lui aussi semblable à une somme gaussienne. La lettre $\gamma$ est employée dans les calculs qui suivent dans le sens des 
formules de [SS]. Ce $\gamma$ est un élément des groupes $C_{\tau}^{h}$, qui sont implicitement des sousgroupes de $S L(2)$, donc des groupes de matrices. L'expression $\operatorname{tr}(\lambda-\gamma)$ est la trace d'une matrice $\lambda-\gamma$. Rappelons aussi que le facteur $S(\psi)$ de [SS] est défini par

$$
S(\psi)=q^{1 / 2} \int_{U} \operatorname{sgn}_{\tau}(x) \psi\left(\frac{1+\sqrt{\tau} t^{h-1} x}{1-\sqrt{\tau} t^{h-1} x}\right) d x,
$$

où, dans la notation de [SS], $U=\{x \in \mathcal{O}|| x \mid=1\}$. Il me semble qu'il y a dans [SS] une incohérence dans les définitions. Selon la définition dans la section $\S 2$ de leur article $d x$ est la mesure sur $F^{\times}$telle que meas $(\mathcal{O})=1$. La mesure implicite dans la formule (1.4.16) telle qu'utilisée ici aussi bien que dans leur article est par conséquent celle pour laquelle $\operatorname{meas}(U)=(q-1) / q$.

L'intégrale (1.4.16) est égale à une somme. Si $|x|=1$, alors

$$
\delta=\frac{1+\sqrt{\tau} t^{h-1} x}{1-\sqrt{\tau} t^{h-1} x}=1+2 \sqrt{\tau} \tau^{h-1} x+2 x^{2} \tau^{2 h-1}+\ldots
$$

est un élément du groupe $C_{\tau}^{h-1}$ de [SS], arbitraire sauf qu'il n'appartient pas à $C_{\tau}^{h}$. On a $\delta=1+\mu \tau^{2 h-1}+\nu \sqrt{\tau} \tau^{h-1}$ avec $\nu \equiv \mu^{2} / 2(\bmod \mathfrak{p})$, où $\mathfrak{p}$ est l'idéal maximal de $\mathcal{O}$. On a $\nu=2 x$ et, selon la définition du conducteur donnée dans [SS], $\psi(\delta)$ ne dépend que de $x$ ou de $\nu$ modulo $\mathfrak{p}$. Il est par conséquent permis de remplacer l'intégrale de (1.4.16) par une somme sur $q-1$ éléments du corps résiduel. Seul $x \equiv 0(\bmod \mathfrak{p})$ est exclu. La mesure est telle que chaque terme de la somme est multiplié par $1 / q$. Le facteur $S(\psi)$ est par conséquent égal à $q^{-1 / 2}$ fois une somme gaussienne

$$
\sum_{x \neq 00(\bmod \mathfrak{p})} \kappa(x) \eta(x)
$$

ou $\kappa$ est le caractère quadratique du groupe multiplicatif du corps résiduel $\mathcal{O} / \mathfrak{p}$ et $\eta$ un caractère non trivial de ce même corps. En particulier, comme on affirme dans [SS], $|S(\psi)|=1$.

Les deux lignes $|1-\lambda|^{2}=q^{-2 h+1}$ et $q^{-2 h+1}<|1-\lambda|^{2}<1$ sont semblables pour les deux tores ramifiés, mais la deuxième ligne est 0 lorsque $T$ n'est pas isomorphe à $H$, donc si $\lambda=\alpha+\sqrt{\epsilon \tau} \beta$. Commençons avec l'examen de la somme des contributions des deux tores données par la première de ces deux lignes. Il serait sans doute utile de rappeler qu'à la suite des formules (1.4.3), (1.4.4), (1.4.5) et (1.4.6), dans lesquelles se trouvent les caractères $\chi_{G}^{\text {st }}$ calculés facilement à partir des formules de [SS], nous calculons les coefficients de $\bar{\psi}$ dans le développement de la distribution $f^{H}$ en utilisant notre hypothèse sur la forme des intégrales orbitales de $f^{G}$, donc sur la forme de $\theta_{f^{G}}^{G}\left(a_{G}\right)$. Un élément essentiel de notre hypothèse est la condition $h \geq m$. C'est elle qui nous permet de vérifier que le coefficient de $\bar{\psi}$ est 0 .

À présent l'hypothèse est que $\alpha_{+}=0$ et $\beta_{+}=1$. Nous calculons l'intégrale de (1.4.4). Notre hypothèse sur les valeurs de $\alpha_{+}$et $\beta_{+}$nous assure que $\theta_{f G}^{G}$ est la fonction caractéristique de l'ensemble $\lambda \in C_{\tau}^{(m)}$, ou plutôt de son image dans la base de SteinbergHitchin. Le groupe $C_{\tau}^{(m)}$ est défini dans [SS]. Comme déjà expliqué, il résulte des formules 
(1.4.4) et (1.4.5) que le coefficient de $\bar{\psi}\left(\gamma_{H}\right)$ dans le développement de Fourier de $f^{H}$ est

$$
\int \theta_{f^{G}}^{G}\left(a_{G}\right)\left|\Delta\left(a_{G}\right)\right| \chi_{\pi_{G}^{\text {st }}}\left(\gamma_{G}\right) d_{\text {geom }} \gamma_{G}
$$

Mais ce $\gamma_{G}$ est le $\lambda$ de [SS], au moins si l'on accepte de confondre la classe de conjugaison d'une matrice et sa valeur propre, elle-même un peu ambiguë. Il est préférable en utilisant les résultats de [SS] de remplacer dans cette formule $\gamma_{G}$ par $\lambda$. L'intégrale se calcule par couronnes, $|1-\lambda|^{2}=q^{-2 l+1}$. Nous avons déjà traité le cas $l>h$ et avons vérifié que pour tout $l, h<l \leq m$ la contribution est 0 . Il reste à traiter les cas $l=h$ et $l \leq h$.

Commençons avec $l=h$ et calculons (1.4.17) sur cette couronne. Nous utilisons la valeur du caractère donnée dans [SS]. Elle devient, après stabilisation, qui le multiplie par 2 et enlève le terme qui contient $H(\Phi, V)$,

$$
\frac{q^{-1 / 2}}{\left|\lambda-\lambda^{-1}\right|} \sum_{\gamma} \operatorname{sgn}_{\tau}(\operatorname{tr}(\lambda-\gamma)) \psi(\gamma)
$$

Le facteur $\left|\Delta\left(a_{G}\right)\right|$ de (1.4.6) enlève le facteur $\left|\lambda-\lambda^{-1}\right|$ du dénominateur de (1.4.18).

Observons que sur cette couronne on peut avoir $\lambda=\alpha^{\prime}+\sqrt{\tau} \beta^{\prime}$ ou $\lambda=\alpha^{\prime}+\sqrt{\epsilon \tau} \beta^{\prime}$. Il faut tenir compte alors de deux lignes différentes du tableau. Mais les formules de [SS] pour ces deux lignes sont les mêmes, sauf que l'indice $\tau$ a par inadvertance été omis dans la première. Les domaines de la sommation sur $\gamma$ sont cependant différents. Dans les deux cas, $\gamma \in F(\sqrt{\tau})=E_{H}$ et $\mathrm{N}_{E_{H} / F} \gamma=1$. En plus, $\gamma \equiv 1\left(\bmod \mathfrak{p}_{E_{H}}^{2 h-1}\right)$ et $\gamma$ n'est donné que modulo $\mathfrak{p}_{E_{H}}^{2 h+1}$. Lorsque $\lambda=\alpha^{\prime}+\sqrt{\tau} \beta^{\prime}$ et $l=h$, alors $\lambda \in C_{\tau}^{(h-1)}$ et l'on peut exiger que $\gamma \not \equiv \lambda^{ \pm 1}\left(\bmod \mathfrak{p}_{E_{H}}^{2 h+1}\right)$, ce qui est fait dans le tableau.

En général, posons $\lambda=\alpha^{\prime}+\sqrt{\tau^{\prime}} \beta^{\prime}$, où $\tau^{\prime}$ est, dans la notation de [SS], soit $\tau$, soit $\epsilon \tau$. Grâce à notre définition des normes, qui est compatible avec celle de [SS], la condition $|1-\lambda|^{2}=q^{-2 h+1}$ est équivalente à $\left|\alpha^{\prime}-1\right| \leq q^{-h},\left|\beta^{\prime}\right|=q^{-h+1}$. Soit $\gamma=\alpha+\sqrt{\tau} \beta$. Pour $\gamma \in C_{\tau}^{(h-1)} / C_{\tau}^{(h)}, \alpha \equiv 1\left(\bmod \mathfrak{p}_{F}^{h}\right)$ est pris modulo $\mathfrak{p}_{F}^{h+1}$ et $\beta \equiv 0\left(\bmod \mathfrak{p}^{h-1}\right)$. Soient $\alpha=1+\mu \tau^{h}, \beta=\nu \tau^{h-1}$. On a

$$
1=\alpha^{2}-\beta^{2} \tau=1+2 \mu \tau^{h}+\mu^{2} \tau^{2 h}-\nu^{2} \tau^{2 h-1},
$$

de sorte que $2 \mu+\nu^{2} \tau^{h-1} \equiv 0\left(\bmod \mathfrak{p}_{F}^{h}\right)$ et $\mu=\mu_{1} \tau^{h-1}, \mu_{1} \in \mathcal{O}_{F}, 2 \mu_{1} \in \mathcal{O}_{F}^{2}$. Puisque nous supposons que $h>1$, cette relation implique que $\mu \equiv 0\left(\bmod \mathfrak{p}_{F}\right)$. Par contre, $\left|\mu_{1}\right|=1$. Une correspondence avec J. Tunnell m'a convaincu que la somme sur $\gamma$ est une somme sur $\gamma \in C_{\tau}^{(h-1)} / C_{\tau}^{(h)}$, que je scinde en deux sommes, une somme sur les classes gauche diffrentes de $C_{\tau(h)}$ et la somme avec un seul terme, celui pour $\gamma=1$.

Commençons avec la somme restreinte, qui n'est donc qu'une somme sur $\nu \not \equiv 0$ modulo $\mathfrak{p}_{F}$. Pour qu'il existe un $\lambda$ tel que $\gamma$ appartient à la même classe à gauche que $\lambda$ ou $\lambda^{-1}$, il est nécessaire (et suffisant) que $\nu \not \equiv 0\left(\bmod \mathfrak{p}_{F}\right)$. Si $\tau=\tau^{\prime}$, alors $\lambda$ appartient à $C_{\tau}^{(h-1)}$ et la condition $\gamma \neq \lambda, \lambda^{-1}$ de [SS] n'est rien que la condition que $\gamma$ n'appartient pas à la classe à gauche dans $C_{\tau}^{(h-1)} / C_{\tau}^{(h)}$ définie par $\lambda$ ou $\lambda^{-1}$. 
Puisque $\left|\tau^{\prime}\right|=\left|q^{-1 / 2}\right|$, la mesure $d \lambda=q^{-1 / 2} d \beta^{\prime}$ et la mesure de l'ensemble des $\lambda$ pour lesquels $\beta^{\prime}$ est donné modulo $\mathfrak{p}_{F}^{h}$ est $q^{-h-1 / 2}$. L'intégrale (1.4.17) devient alors une somme sur $\nu$ et $\nu^{\prime}, \beta^{\prime}=\nu^{\prime}\left(\tau^{\prime}\right)^{n-1}$, à savoir

$$
q^{-h-1} \sum_{\substack{\nu, \nu^{\prime} \\ \nu, \nu^{\prime} \neq 0\left(\bmod \mathfrak{p}_{F}\right) \\ \nu \neq \equiv \pm \nu^{\prime}\left(\bmod \mathfrak{p}_{F}\right)}} \operatorname{sgn}_{\tau}(\operatorname{tr}(\lambda-\gamma)) \psi(\gamma)
$$

On peut calculer le nombre de paires qui interviennent dans cette somme de deux façons. Il y a $q-1$ possibilités pour $\gamma$ modulo $C_{\tau}^{(h)}$ et pour chacune de ses possibilités, il y a $q-3$ possibilités pour $\lambda \in C_{\tau^{\prime}}^{(h-1)}$ modulo $C_{\tau^{\prime}}^{(h)}$ si $\tau^{\prime}=\tau$, car $\nu^{\prime} \equiv 1$ et $\lambda \equiv \gamma^{ \pm 1}$ sont exclus, et il y en a $q-1$ si $\tau^{\prime}=\epsilon \tau$. Donc, somme toute $2(q-1)(q-2)$ possibilités. D'autre part, il y a $2(q-1)$ possibilités pour $\lambda$ et pour chacune des $q-2$ possibilités pour $\gamma$, car $\gamma \neq \lambda^{ \pm 1}$. Observons aussi qu'en passant de $\lambda$ à $\lambda^{-1}$ ou de $\gamma$ à $\gamma^{-1}$, on passe de $\nu^{\prime}$ à $-\nu^{\prime}$ ou de $\nu$ à $-\nu$.

Considérons d'abord la somme

$$
\sum_{\gamma} \psi(\gamma) \sum_{\lambda} \operatorname{sgn}_{\tau}(\operatorname{tr} \lambda-\operatorname{tr} \gamma)
$$

qui est une somme sur $\lambda$ et $\gamma$ avec les conditions décrites. Écrivons

$$
\alpha^{\prime}=1+\mu_{1}^{\prime}\left(\tau^{\prime}\right)^{2 h-1}, \quad \beta^{\prime}=\nu^{\prime}\left(\tau^{\prime}\right)^{h-1},
$$

de sorte que $\operatorname{tr} \lambda=2+2 \mu_{1}^{\prime}\left(\tau^{\prime}\right)^{2 h-1}$. Puisque nous pouvons choisir $\tau^{\prime}$ égal à $\tau$ ou à $\epsilon \tau$, il est possible et préférable de poser $\operatorname{tr} \lambda=2+2 \mu_{1}^{\prime} \tau^{2 h-1}$ où le facteur $\mu_{1}^{\prime}$ parcourt le groupe multiplicatif $\left(\mathcal{O}_{F} / \mathfrak{p}_{F}\right)^{\times}$, sauf qu'il faut omettre $\mu_{1}^{\prime} \equiv \mu_{1}$ qui correspond à $\lambda=\gamma^{ \pm 1}$. Observons que pour une valeur donnée de $\mu_{1}^{\prime}$ il y a deux valeurs possible de $\nu_{1}^{\prime}$. Puisque $\operatorname{sgn}_{\tau}\left(\tau^{2 h-1}\right)=\operatorname{sgn}_{\tau}(\tau)$, la somme intérieure de (1.4.20) est égale à

$$
2 \sum_{\mu_{1}^{\prime}} \operatorname{sgn}_{\tau}\left(2+2 \mu_{1}^{\prime} \tau^{2 h-1}-2-2 \mu_{1} \tau^{2 h-1}\right)=2 \operatorname{sgn}_{\tau}(\tau) \sum \operatorname{sgn}_{\tau}\left(2\left(\mu_{1}^{\prime}-\mu_{1}\right)\right) .
$$

Dans la somme à droite, la condition est que $\mu_{1}^{\prime}$ n'est congruent ni à 0 ni à $\mu_{1}$ modulo $\mathfrak{p}_{F}$.

En mettant $\mu_{2}=\mu_{1}^{\prime}-\mu_{1}$ et en permettant la valeur $\mu_{2}=-\mu_{1}$, nous déduisons que le côté gauche de (1.4.22) est égal à la somme de

$$
2 \operatorname{sgn}_{\tau}(\tau) \sum_{\mu_{2} \neq 0} \operatorname{sgn}_{\tau}\left(2 \mu_{2}\right)=0
$$

et de

$$
-2 \operatorname{sgn}_{\tau}(\tau) \operatorname{sgn}_{\tau}\left(-2 \mu_{1}\right) .
$$


Nous avons déjà observé que $2 \mu_{1}$ est nécessairement un carré. Par conséquent (1.4.23) est égal à $-2 \operatorname{sgn}_{\tau}(-\tau)=-2$, donc indépendant de $\gamma$. Puisque le conducteur de $\psi$ est $h$, la somme sur $\gamma \in C_{\tau}^{(h-1)} / C_{\tau}^{(h)}, \gamma \notin C_{\tau}^{(h)}$, est $-\psi(1)=-1$. Par conséquent (1.4.19) est égal à $2 q^{-h-1}$ et cette contribution enlève les deux contributions (1.4.15).

Pour le terme supplémentaire $\gamma=1$, plus précisement $\gamma \in C_{\tau}^{(h)}$, on a encore la somme (1.4.22) mais avec $\mu_{1}=0$ ou $\mu_{1} \equiv 0$ et la condition sur $\lambda$ est $\mu_{1}^{\prime} \not \equiv 0$, de sorte que l'on obtient la somme supplémentaire nulle

$$
2 \operatorname{sgn}_{\tau}(\tau) \sum_{\mu_{1}^{\prime} \neq 0} \operatorname{sgn}_{\tau}\left(\mu_{1}^{\prime}\right)=0
$$

Donc cela ne modifie pas la conclusion. Il faut néanmoins tenir compte de ce terme en examinant le transfert $f^{G} \mapsto f^{H}$, ce qu'il faut faire tôt ou tard.

Il reste des contributions des couronnes $|1-\lambda|^{2}=q^{-2 k+1}, 0<k<h$. Quoiqu'il n'y a plus de $\gamma$ nous n'omettrons pas les primes et poserons $\lambda=\alpha^{\prime}+\sqrt{\tau} \beta^{\prime}$; la possibilité $\tau^{\prime}=\epsilon \tau$ ne donne plus de contribution. Pour la couronne on a $\alpha^{\prime}=1+\mu^{\prime} \tau,\left|\mu^{\prime}\right|=1, \beta^{\prime}=\nu^{\prime} \tau^{k-1}$, $\left|\nu^{\prime}\right|=1$. La contribution est décrite par une seule ligne du premier tableau. La formule donnée se simplifie après stabilisation. Le facteur $1 / 2$ est enlevé par stabilisation aussi bien que les termes qui contiennent $H(\Phi, V)$. Le facteur est

$$
\operatorname{sgn}_{\tau}\left(\frac{\lambda-\lambda^{-1}}{2 \sqrt{\tau}}\right)=\operatorname{sgn}_{\tau}\left(\beta^{\prime}\right)=\operatorname{sgn}_{\tau}\left(\nu^{\prime}\right) \operatorname{sgn}_{\tau}(-1)^{k-1}
$$

Observons qu'au contraire du cas non ramifié, pour le cas ramifié $\operatorname{sgn}_{\tau}\left(\beta^{\prime}\right)$ n'est pas constant sur une couronne donnée. La fonction qu'il faut intégrer sur la couronne est par conséquent

$$
\operatorname{sgn}_{\tau}\left(\nu^{\prime}\right)\left\{\operatorname{sgn}_{\tau}(-1)^{k+h} S(\psi) \psi(\lambda)+\operatorname{sgn}_{\tau}(-1)^{k+h-1} S(\psi) \psi\left(\lambda^{-1}\right)\right\}
$$

On a les couronnes $|1-\lambda|^{2}=q^{-2 k+1}$ et les disques $|1-\lambda|^{2} \leq q^{-2 k^{\prime}+1}$. Si $k<k^{\prime} \leq h$, en particulier si $k^{\prime}=h$, le produit d'un élément $\lambda$ de la couronne et un élément $\lambda^{\prime}$ du disque appartient encore à la couronne. Pour un $\lambda^{\prime}$ donné cette action sur le disque ne change pas la mesure, mais elle peut changer la valeur de $\psi(\lambda)$ qui n'est pas nécessairement égale à $\psi\left(\lambda \lambda^{\prime}\right)$, car le plus grand disque sur lequel $\psi$ est constant est donné par $k^{\prime}=h+1$. L'intégrale est donc 0 si $k<h$. Donc pour démontrer que l'intégrale est 0 sur la couronne $|1-\lambda|^{2}=q^{-2(h-1)+1}$, il suffit de démontrer qu'elle est 0 sur le disque de rayon $q^{-2(h-1)+1}$

Ce qui reste pour donner la réponse complète à la question $\mathrm{B}$ dans le cas des tores ramifiés est le cas $\alpha_{+}=1, \beta_{+}=0$. Le tore déployé ne contribue rien. La contribution du tore non ramifié et du tore ramifié se calcule de la même façon que les contributions pour $\alpha_{+}=0, \beta_{+}=1$, en ajoutant les facteurs $-c_{\text {unr }} / d_{\text {unr }}$ ou $-c_{\text {ram }} / d_{\text {ram }}$ aussi bien qu'un facteur $|\Delta(t)|$. Les deux premiers facteurs sont des constantes; le dernier est constant sur des couronnes, de façon que les résultats des calculs précédents qui ont été fais par couronnes s'appliquent tels quels. 
En modifiant la somme (1.4.14) selon ces observations nous obtenons d'abord la contribution du tore ramifié. Elle est

$$
-2 q^{h-1}(1-1 / q) \sum_{k=h}^{\infty} q^{-3 k}=-2 q^{-2 h-1} \frac{1}{1+1 / q+1 / q^{2}} .
$$

La contribution des tores ramifiés est la somme de deux termes. Le premier, qui est obtenu en modifiant (1.4.15) et en se rappelant que $|\Delta(\lambda)|=q^{-k-1 / 2}$, est une somme sur les petites couronnes, à savoir

$$
-2 q^{h-1 / 2}(1+1 / q) \sum_{k=h}^{\infty}(1-1 / q) q^{-3 k-3 / 2}=-2 q^{-2 h-2} \frac{1+1 / q}{1+1 / q+1 / q^{2}} .
$$

La somme de (1.4.25) et (1.4.26) est $-2 q^{-2 h-1}$. Puisque les grandes couronnes $|1-\lambda|=$ $q^{-2 k+1}, k<h$, contribuent 0 , il ne reste que la contribution de la couronne $|1-\lambda|=q^{-2 h+1}$. Elle est le produit de (1.4.19), de $q^{-1 / 2}$ et de $q^{-h+1 / 2}$, donc égale à $2 q^{-2 h-1}$. Somme toute, le résultat est 0 .

\section{Les singularités}

2.1. Les singularités des fonctions $\theta(\cdot)$ pour un corps archimédien. Nous rappelons au début de la $\S 3$ de cet article qu'à nos fins il suffit de traiter les fonctions $\theta^{G}\left(a_{G}, 1\right)=$ $\theta^{G}\left(a_{G}\right)$, pour lesquelles nous avons mis $s=1$. Rappelons que

$$
\theta^{G}\left(a_{G}\right)=\left|\Delta^{G}\left(a_{G}\right)\right| \int f^{G}\left(g^{-1} \gamma g\right) \frac{d_{\mathrm{geom}} g}{d_{\text {geom }} t}
$$

où nous avons omis l'indice $v$, mais où la notation est, à part cela, celle de la section $\S 3$ de $[\mathrm{FLN}]$, donc un peu fastidieuse en ce qui concerne les facteurs $L$. La constante $L_{v}\left(1, \sigma_{T / G}\right)$ de la formule (3.31) de [FLN] a pour effet de remplacer la mesure normalisée $d \bar{g}_{v}$ par le quotient des mesures géométriques. La présence, implicite ou explicite, de ce facteur est évidemment clé dans certains énoncés, comme, par exemple, le lemme 2.1.4. Pour tout corps local et tout groupe réductif, les intégrales orbitales sont fondamentales pour l'analyse harmonique invariante. Pour un corps archimédien elles ont été traitées par Harish-Chandra ([V]). Pour le groupe $S L(2)$, je préfère citer [Kn] car en ne traitant que $S L(2)$ il pouvait offrir des explications plus brèves et plus élémentaires.

D'abord pour $S L(2)$ sur le corps $\mathbb{C}$ il n'y a qu'une seule classe de sous-groupes de Cartan, le tore déployé. Comme représentant on peut choisir le tore diagonal comme fait Knapp dans $§ X I .2$ de $[\mathrm{Kn}]$. Pour lui un élement typique de ce groupe est

$$
\gamma=\left(\begin{array}{cc}
e^{u+i \theta} & 0 \\
0 & e^{-u-i \theta}
\end{array}\right)
$$

Puisque la valeur absolue de $z \in \mathbb{C}$ est $z \bar{z}$, on a

$$
|\Delta(\gamma)|=\left(e^{u+i \theta}-e^{-u-i \theta}\right)\left(e^{u-i \theta}-e^{-u+i \theta}\right),
$$


et à une constante près,

$$
\theta^{G}\left(a_{G}\right)=F_{f}^{T}(\gamma), \quad \mathbf{c}(\gamma)=a_{G},
$$

où $F_{f}^{T}$ est la fonction de la formule (11.13) de [Kn]. Elle est une fonction lisse à support compact. Donnons un énoncé formel pour pouvoir y renvoyer le lecteur.

Lemme 2.1.2. Si $F=\mathbb{C}$ et si $f=f^{G}$ est lisse à support compact, alors la fonction $f^{H}$ de (1.2.6) est lisse à support compact.

Pour le corps réel il y a le tore déployé et le tore elliptique. Des représentants de ces deux tores sont donnés dans les formules (1.3.1). Pour les définitions de [Kn, §X.2] comme pour celles de [FLN] il faut choisir des mesures de Haar sur les tores et sur le groupe $G$, ou plutôt sur l'ensemble de leurs points à coordonnées dans $F$. Cela donne pour chacun de ces groupes deux mesures de Haar qui diffèrent d'une constante. Si nous suivons [Kn] en dénotant le tore déployé de (1.3.1) par $T=T_{\mathrm{sp}}$ et le tore compact par $B$, ceci donne trois constantes $c_{G}, c_{T}$ et $c_{B}$. La constante $c_{G}$ n'est pas pour le moment importante, mais pour vérifier l'existence du transfert sur le corps $\mathbb{R}$ en utilisant les formules de $[\mathrm{Kn}]$ il faut bien tenir compte des valeurs de $c_{T}$ et de $c_{B}$.

Pour $S L(2)$ ou n'importe quel groupe de matrices la matrice $g^{-1} d g$ de formes de degré 1 est certainement invariante à gauche. Pour $S L(2)$,

$$
g^{-1} d g=\left(\begin{array}{ll}
d \alpha & d \beta \\
d \gamma & d \delta
\end{array}\right)
$$

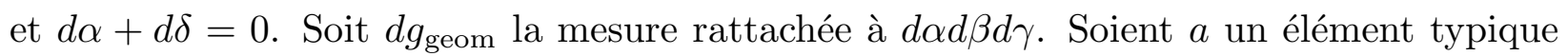
du tore déployé et $s$ un élément typique du tore compact. Les mesures géométriques sur ces deux groupes sont $d_{\text {geom }} a=d t / t, a= \pm a(t), t>0, d_{\text {geom }} s=d \theta, s=s(\theta)$. Nous dénotons les mesures définies dans la section $\S \mathrm{X} .2$ de $[\mathrm{Kn}]$ par $d^{K} g, d^{K} a$ et $d^{K} s$. Il utilise la décomposition de Iwasawa

$$
g=\left(\begin{array}{cc}
\cos \theta & \sin \theta \\
-\sin \theta & \cos \theta
\end{array}\right)\left(\begin{array}{ll}
1 & x \\
0 & 1
\end{array}\right)\left(\begin{array}{cc}
e^{t} & 0 \\
0 & e^{-t}
\end{array}\right)
$$

pour définir $d^{K} g=d \theta d x d t / 2 \pi$. Sur $T$ il utilise $d^{K} t=d t$ (en tenant compte des différences de notation, c'est la mesure $d_{\text {geom }} a, a=a(t)$ de [FLN]) et sur $B$ il utilise $d^{K} s=d \theta / 2 \pi$ (c'est maintenant la mesure $d$ geom $s(\theta) / 2 \pi$ ). On vérifie facilement qu'en l'élément neutre

$$
d \alpha d \beta d \gamma=d \theta d x d t
$$

de sorte que $d^{K} g=d_{\text {geom }} g / 2 \pi$. Il en résulte que $c_{T}=1$ et $c_{B}=2 \pi$.

Il faut ne pas oublier que les intégrales orbitales utilisées dans [Kn] et données par les équations (10.9a) et (10.9b) du livre ne sont pas des intégrales stables. Celle pour le tore déployé est de toute façon stable et on a

$$
\theta^{G}\left(a_{G}\right)= \pm c_{G} F_{f}^{T}( \pm a(t)), \quad \pm a(t) \mapsto a_{G}= \pm\left(e^{t}+e^{-t}\right)
$$

Observons en particulier que nous avons 
Lemme 2.1.3. Si $F=\mathbb{R}$, si $f=f^{G}$ est lisse à support compact, et si $H$ est le tore déployé, alors la fonction $f^{H}$ de (1.2.6) est lisse à support compact.

Pour obtenir une intégrale stable pour le tore $B$ il faut utiliser la différence $F_{f}^{B}(\theta)$ $F_{f}^{B}(-\theta)$, car

$$
\theta^{G}\left(a_{G}\right)=\frac{i \operatorname{sgn}(\sin \theta) c_{G}}{\pi}\left(F_{f}^{B}(\theta)-F_{f}^{B}(-\theta)\right), \quad s(\theta) \mapsto a_{G}=2 \cos \theta .
$$

L'indice $G$ est utile pour distinguer la fonction $\theta^{G}$ de la variable $\theta$, qui n'intervient que dans les renvois à $[\mathrm{Kn}]$. Observons que la fonction à droite est une fonction paire comme il se doit.

Selon Lemma 10.10 de [Kn], qui est l'énoncé pour $S L(2)$ d'un lemme général trouvé et utilisé par Harish-Chandra, la fonction $F_{f}^{T}( \pm a(t))$ est pour chacun des choix de signes une fonction lisse de $t$ ou de $e^{t}$ à support compact. Les formules (11.36a) et (11.36b) s'écrivent

$$
F_{f}^{B}\left(0^{+}\right)-F_{f}^{B}\left(0^{-}\right)=\pi i F_{f}^{T}( \pm a(0)),
$$

si à gauche $\theta=0, \pi$ et à droite $\pm a(0)=\cos (\theta)$. (Dans l'équation (11.36b), il manque un signe, comme on voit en remplaçant $f(g)$ par $f(-g)$.)

La fonction $\theta^{G}$ n'est pas définie aux points $a_{G}= \pm 2$, mais sa valeur en ces deux points peut être définie à partir des équations (2.1.2) et (2.1.3).

$$
\begin{aligned}
\theta^{G}( \pm 2) & = \pm c_{G} F_{f}( \pm a(0)), \\
\theta^{G}(2) & =\lim _{\theta \rightarrow 0} \frac{i c_{G}}{\pi}\left(F_{f}^{B}\left(0^{+}\right)-F_{f}^{B}\left(0^{-}\right)\right), \\
\theta^{G}(-2) & =\lim _{\theta \rightarrow \pi} \frac{i c_{G}}{\pi}\left(F_{f}^{B}\left(\pi^{+}\right)-F_{f}^{B}\left(\pi^{-}\right)\right) .
\end{aligned}
$$

Il résulte de la formule (2.1.4) que $\theta^{G}$ est bien défini et continu en $a_{G}= \pm 2$ et par conséquent partout.

Il n'est pas toutefois lisse en ces deux points. Son comportement est décrit dans le prochaine lemme. Soit $\pm a_{G}=2+x$.

Lemme 2.1.4. Si $F=\mathbb{R}$, dans un voisinage de $x=0$, la fonction $\theta^{G}$ est une somme $\theta^{G}=\theta_{e}^{G}+\theta_{o}^{G}$, où $\theta_{e}^{G}$ a un développement asymptotique en $x=0$,

$$
\theta_{e}^{G}(x) \sim \sum_{n=0}^{\infty} a_{n}^{ \pm} x^{n},
$$

et où $\theta_{o}^{G}$ est 0 pour $x>0$, mais où pour $x<0$, donc pour $\left|a_{G}\right|<2$, il y a le développement asymptotique

$$
\theta_{o}^{G}(x) \sim \sum_{n=0}^{\infty} b_{n}^{ \pm}|x|^{n+1 / 2} .
$$


La formule (2.1.6) est valable des deux côtés de $x=0$. Il résulte donc du lemme que $\theta^{G}$ est la somme d'une fonction lisse dont les dérivées sont données par (2.1.6) et d'une fonction égale à 0 pour $x>0$, mais dont les singularités pour $x<0$ sont données par (2.1.7). Le lemme résulte de la $\$ 11.3$ de $[\mathrm{Kn}]$. Expliquons.

Un simple calcul montre, comme dans $[\mathrm{Kn}]$, que $\pm c_{G} F_{f}^{T}( \pm a(t))$ est une fonction lisse et paire de $t$ dont l'expansion formelle autour de 0 s'écrit

$$
\sum_{n=0}^{\infty} A_{n}^{ \pm} t^{2 n}
$$

En plus,

$$
\pm a_{G}=2+2 t^{2}+\sum_{m=2}^{\infty} \frac{2}{(2 m) !} t^{2 m}=2+x .
$$

Donc $\left|a_{G}\right| \geq 2, x \geq 0$ et, en posant $\alpha_{1}=1 / 2$, nous obtenons

$$
t^{2}=\frac{x}{2}+\sum_{m=2}^{\infty} \alpha_{m} x^{m}=\sum_{m=1}^{\infty} \alpha_{m} x^{m}
$$

Il en résulte que

$$
\theta^{G}\left(a_{G}\right)= \pm c_{G} F_{f}^{T}( \pm a(t)) \sim \sum_{0}^{\infty} a_{m}^{ \pm} x^{m}
$$

où chaque $a_{m}^{ \pm}$est une fonction linéaire des $A_{n}$ à coefficients qui sont des polynômes en les $\alpha_{m}$. Supposons qu'un terme $A_{n} \alpha_{1}^{d_{1}} \ldots \alpha_{l}^{d_{l}}$ intervient avec un coefficient non nul. Alors

$$
m=n+d_{1} m_{1}+\ldots d_{l} m_{l} .
$$

La relation (2.1.10) affirme simplement que la fonction $\theta^{G}\left(a_{G}\right)$ est lisse en $a_{G}= \pm 2$ et que ces dérivées sont calculées à partir des coefficients $a_{n}^{ \pm}$. Elle définit donc ces coefficients.

Si nous remplaçons $\pm a(t)$ par $s(\theta)$ ou par $s(\theta+\pi)$ et si, dans les deux cas, $\theta$ reste dans un voisinage de 0 , alors

$$
\pm a_{G}=e^{i \theta}+e^{-i \theta}=2+\sum_{m=1}^{\infty} \frac{2}{(2 m) !}(-1)^{m} \theta^{2 m}=2+y, \quad y<0
$$

Évidemment, l'équation (2.1.9) devient

$$
-\theta^{2}=\sum_{m=1}^{\infty} \alpha_{m} y^{m}
$$


Supposons que la fonction de (2.1.3) a un développement formel,

$$
\theta^{G}\left(a_{G}\right) \sim \sum_{n=0}^{\infty}(-1)^{n} A_{n}^{ \pm} \theta^{2 n}+\sum_{n=0}^{\infty} B_{n}^{ \pm} \theta^{2 n+1}
$$

avec les coefficients de (2.1.8). Alors en utilisant (2.1.12) et la modification de (2.1.11) que cela entraîne, nous déduisons que la relation (2.1.6) est valable si nous utilisons la première somme de (2.1.13) pour définir $\theta_{e}^{G}$ dans le domain $x<0$, donc là où la coordonnée est $y$.

Il faut avouer que nous n'avons pas encore défini les fonctions $\theta_{e}^{G}$ et $\theta_{o}^{G}$. Le faire est facile. Il s'agit de définir une fonction lisse avec des dérivées données à un seul point, mais c'est le développement formel donné par le lemme qui est important, et nous l'avons vérifié sauf que la relation (2.1.13) reste à établir.

Donc les variables que nous voulons employer sont $x>0$ et $y<0$, qui ne sont que deux possibilités pour la coordonnée dans un voisinage de $a_{G}= \pm 2$. Dans les formules et des théorèmes habituels, qui sont aussi ceux de $[\mathrm{Kn}]$ on utilise toutefois $t$ ou $\theta$. Ces théorèmes nous donnent d'abord le développement (2.1.8). Les formules (11.34a) et (11.34b) donnent le premier coefficient de (2.1.13), le terme constant. Grâce à la formule (2.1.3) et à la formule (11.35) de $[\mathrm{Kn}]$ nous savons que la limite de sa dérivée par rapport à $\theta$ existe et que $B_{1}^{ \pm}=4 c_{G} f^{G}( \pm 1)$, donc le produit de $4 c_{G}$ et de la valeur de $f^{G}$ à un des deux éléments centraux de $G(F)$.

Pour terminer, nous avons besoin de quelques formules bien connues de la théorie des opérateurs différentiels invariants sur des groupes de Lie semi-simples telle que créée et utilisée par Harish-Chandra. Pour cette théorie nous renvoyons le lecteur à l'introduction à ses travaux complets écrite par Varadarajan. Ce qu'il est pertinent de savoir ici, c'est qu'il y a un opérateur différentiel invariant $\varpi$ tel qu'en remplaçant $f$ par $\varpi^{n} f$, nous remplaçons $F_{f}^{T}$ par $d^{2 n} F_{f}^{T} / d t^{2 n}$ et $F_{f}^{B}$ par $(-1)^{n} d^{2 n} F_{f}^{B} / d \theta^{2 n}$. En passant à $\varpi f$ nous obtenons le résultat voulu pour $A_{1}^{ \pm}$et $B_{1}^{ \pm}$, à $\varpi^{2} f$, pour $A_{2}^{ \pm}$et $B_{2}^{ \pm}$, et ainsi de suite.

2.2. Les singularités des fonctions $\theta(\cdot)$ pour un corps $p$-adique. Nous avons observé dans la formule $(2.1 .1)$ que la mesure $d_{\text {geom }} g / d_{\text {geom }} t$ donne une définition de $\theta^{G}$ dans laquelle le facteur $L\left(1, \sigma_{T / G}\right)$ n'apparaît pas explicitement. Nous l'utilisons aussi pour les corps $p$-adiques. Le lemme 2.1.4 décrit pour $S L(2)$ le comportement asymptotique de $\theta^{G}\left(a_{G}\right)$ aux points $a_{G}= \pm 2$. Il peut être intérprété aussi comme une description des intégrales orbitales stables. Pour un corps $p$-adique les premières informations sur le comportement de ces intégrales se trouvent dans [Sh, Th. 2.2.2]. Pour le groupe $S L(2)$, au moins pour $p \neq 2$, une hypothèse que nous avons déjà admise dans la section $\S 1.4$, le deuxième théorème de cet article nous assure que pour un sous-groupe de Cartan $T$ et pour $t$ dans un voisinage d'un élément central $\pm I$,

$$
\theta^{G}\left(a_{G}\right)=-c_{T}|\Delta(t)| \alpha_{ \pm}+d_{T} \beta_{ \pm}, \quad t \rightarrow a_{G}
$$

Comme toujours $\theta^{G}=\theta_{f^{G}}^{G}$. Les constantes $\alpha_{ \pm}=\alpha_{ \pm}\left(f^{G}\right), \beta_{ \pm}=\beta_{ \pm}\left(f^{G}\right)$ dépendent de $f^{G}$ mais les $c_{T}$ et les $d_{T}$ ne dépendent que de la classe stable du tore. En fait, ils ne dépendent 
que du type du tore: déployé, non ramifié et ramifié. On a donc six constantes: $c_{\mathrm{sp}}, c_{\mathrm{unr}}$, $c_{\mathrm{ram}}$ et $d_{\mathrm{sp}}, d_{\mathrm{unr}}, d_{\mathrm{ram}}$. Nous avons mis $\alpha_{ \pm}=f^{G}( \pm I)$ et

$$
\begin{gathered}
\beta_{ \pm}=\int_{N\left(F_{v}\right) \backslash G\left(F_{v}\right)} f\left( \pm g^{-1} \nu g\right), \\
\nu=\left(\begin{array}{ll}
1 & 1 \\
0 & 1
\end{array}\right) .
\end{gathered}
$$

Pour fixer la mesure sur le quotient, il faut fixer une forme sur $N\left(F_{v}\right)$. Prenons

$$
d n=d x, \quad n=n(x)=\left(\begin{array}{ll}
1 & x \\
0 & 1
\end{array}\right)
$$

La forme sur $G$ qui définit la mesure $d_{\text {geom }}$ a été introduite dans la section précédente.

Shalika a calculé les constantes $c_{T}$ et $d_{T}$, ce qui n'est pas difficile, mais je ne connais pas de référence. Puisque nous en avons besoin, j'explique une façon de faire le calcul. Je suppose toujours que la caractéristique résiduelle n'est pas 2.

Lemme 2.2.2. On a les égalités

$$
\begin{gathered}
d_{\mathrm{sp}}=d_{\mathrm{unr}}=d_{\mathrm{ram}}=\left(1+1 / q_{v}\right) \mathrm{Nd}^{-1}, \\
c_{\mathrm{sp}}=0, \quad c_{\mathrm{unr}}=\frac{2}{q_{v}} \mathrm{No}^{-1}, \quad c_{\mathrm{ram}}=q_{v}^{-1 / 2}\left(1+1 / q_{v}\right) \mathrm{No}^{-1} .
\end{gathered}
$$

Ce lemme est l'analogue pour les corps $p$-adiques du lemme 2.1.4. Quoique dans les deux cas, il s'agit de lemmes dont il doit exister des formes valables pour n'importe quel groupe réductif, nous donnons encore une démonstration à partir des calculs particuliers, en utilisant Lemma 1 de [L2]. Le facteur $\mathrm{No}^{-1}$ fut défini dans la section $\S 3$ de [FLN]. Il est 1 si la mesure locale sur $F_{v}$, à partir de laquelle toutes les mesures géométriques locales sont définies, donne la mesure 1 à $\mathcal{O}_{v}$. C'est ce que nous supposons.

Soit $G^{\prime}=G L(2)$. Si $T$ est un sous-groupe de Cartan de $S L(2)$, son centralisateur est un sous-groupe de Cartan de $G^{\prime}$. L'ensemble $T(F) \backslash G(F)$ est un sous-ensemble de $T^{\prime}(F) \backslash G^{\prime}(F)$. Ils sont égaux si $T$ est déployé, sinon, si $E / F$ est l'extension quadratique rattachée à $T$, alors $T^{\prime}(F) \backslash G^{\prime}(F)=T(F) \backslash G(F) \cup T(F) \backslash G(F) g$, où $g$ est tel que det $g \notin$ $\mathrm{N}_{E / F} E^{\times}$. Si $f^{\prime}$ est un fonction lisse à support compact sur $G^{\prime}(F)$ et $f$ sa restriction à $G(F)$, alors

$$
\operatorname{Orb}\left(\gamma, f^{\prime}\right)=\operatorname{Orb}^{\mathrm{st}}(\gamma, f), \quad \gamma \in T(F)
$$

pourvu que les mesures sur les deux quotients sont compatibles. 
La puissance $p^{-k}$ de [L2] est remplacée par $q_{v}^{-k}$ et

$$
\Delta(\gamma)= \begin{cases}q_{v}^{-k} & \text { si } T \text { est déployé } \\ q_{v}^{-k} & \text { si } T \text { est non ramifié } \\ q_{v}^{-k-1 / 2} & \text { si } T \text { est ramifié. }\end{cases}
$$

Les valeurs du produit de $\Delta(\gamma)$ et des intégrales orbitales données par le lemme de [L2] sont alors

$$
\begin{cases}1 & \text { si } T \text { est déployé, } \\ \frac{q_{v}+1}{q_{v}-1}-\Delta(\gamma) \frac{2}{q_{v}-1} & \text { si } T \text { est non ramifié, } \\ \frac{q_{v}^{1 / 2}}{q_{v}-1}-\Delta(\gamma) \frac{1}{q_{v}-1} & \text { si } T \text { est ramifié. }\end{cases}
$$

Dans le lemme il y a un entier $m \geq 0$ à fixer. Supposons que $m=0$ de sorte que la fonction $f^{\prime}=U^{m}$ est la fonction caractéristique de $G^{\prime}(\mathcal{O})$, et sa restriction $f$, celle de $G(\mathcal{O})$. La condition sur $\gamma$ est alors que ses valeurs propres $\gamma_{1}, \gamma_{2}$ sont des entiers et que leur produit est 1 .

Les mesures de [L2] ne sont pas toutefois celles de cet article. Dans cet article il faudra choisir les mesures géométriques d'une façon cohérente. Sur $G$ nous avons choisi $d_{\text {geom }} g=d \alpha d \beta d \gamma$ et sur un tore nous avons choisi $d_{\text {geom }} \gamma=d \gamma_{1} / \gamma_{1}$. Plus précisément, nous avons choisi les mesures rattachées à ces formes par la mesure invariante sur $F_{v}$ telle que $\operatorname{mes}(\mathcal{O})=1$. Si nous choisissons sur le groupe $G^{\prime}$ la forme

$$
\frac{d \alpha d \beta d \gamma d(\alpha \delta-\beta \gamma)}{\alpha \delta-\beta \gamma}=\frac{\alpha}{\alpha \delta-\beta \gamma} d \alpha d \beta d \gamma d \delta
$$

et sur un tore la forme

$$
\frac{d\left(\gamma_{1} \gamma_{2}\right) d \gamma_{2}}{\gamma_{1} \gamma_{2}^{2}}=\frac{d \gamma_{1} d \gamma_{2}}{\gamma_{1} \gamma_{2}}
$$

nous aurons la compatibilité exigée pour (2.2.5). Cela mène aux mesures

$$
\begin{aligned}
& \operatorname{mes} G(\mathcal{O})=\left(1-\frac{1}{q_{v}}\right)\left(1-\frac{1}{q_{v}^{2}}\right), \\
& \operatorname{mes} T(\mathcal{O})= \begin{cases}\left(1-\frac{1}{q_{v}}\right)^{2} & \text { si } T \text { est déployé, } \\
\left(1-\frac{1}{q_{v}^{2}}\right) & \text { si } T \text { est non ramifié, } \\
q_{v}^{-1 / 2}\left(1-\frac{1}{q_{v}}\right) & \text { si } T \text { est ramifié. }\end{cases}
\end{aligned}
$$


où par $T(\mathcal{O})$ nous comprenons l'ensemble de $\gamma \in T(\mathcal{O})$ tels que les valeurs propres $\gamma_{1}, \gamma_{2}$ sont entières, aussi bien que leurs inverses. Donc pour arriver aux valeurs des constantes du lemme, il faut multiplier les formules (2.2.7) par

$$
\begin{cases}1+\frac{1}{q_{v}} & \text { si } T \text { est déployé, } \\ 1-\frac{1}{q_{v}} & \text { si } T \text { est non ramifié, } \\ q_{v}^{1 / 2}\left(1-\frac{1}{q_{v}^{2}}\right) & \text { si } T \text { est ramifié, }\end{cases}
$$

ce qui donne

$$
\begin{cases}1+\frac{1}{q_{v}} & \text { si } T \text { est déployé, } \\ 1+\frac{1}{q_{v}}-\frac{2}{q_{v}}|\Delta(\gamma)| & \text { si } T \text { est non ramifié } \\ 1+\frac{1}{q_{v}}-q_{v}^{-1 / 2}\left(1+\frac{1}{q_{v}}\right)|\Delta(\gamma)| & \text { si } T \text { est ramifié, }\end{cases}
$$

comme affirmé dans le lemme.

Nous vérifions la dernière des formules (2.2.9). Les deux racines $\gamma_{1}$ et $\gamma_{2}$ de $\gamma$ s'écrivent $\alpha+\beta \varpi, \alpha-\beta \varpi$, où $\varpi^{2} \in F$ et $\left|\varpi^{2}\right|=q_{v}^{-1}$. Par conséquent,

$$
\frac{d \gamma_{1} d \gamma_{2}}{\gamma_{1} \gamma_{2}}=\frac{d \alpha+\varpi d \beta}{d \alpha-\varpi d \beta} \gamma_{1} \gamma_{2}=-\frac{2 \varpi}{\gamma_{1} \gamma_{2}} d \alpha d \beta
$$

Puisque $q_{v}$ est impair et que $|\alpha|=1,|\beta| \leq 1$ est la condition pour l'intégralité de $\gamma$ et de son inverse, la formule s'ensuit. Quoique les normalisations des mesures et des définitions des intégrales orbitales et des fonctions $\theta_{f}$ ont été choisies soigneusement, je m'y perds constamment. Pour ne déboussoler ni moi-même ni le lecteur, je rappellerai plus souvent que nécessaire les conventions.

2.3. Les singularités des fonctions $\varphi(\cdot)$. Les fonctions $\varphi$, ou $\varphi_{v}=\varphi_{f_{v}}$ car elles sont d'abord locales, ne sont pas encore définies. Elles sont définies en premier lieu pour les corps locaux, pour les corps archimédiens aussi bien que pour les corps non archimédiens, à partir d'une fonction $f_{v}^{G}$ en utilisant les fonctions $f_{v}^{H}$ rattachées à elles. La fonction $\varphi$ globale rattachée à $f^{G}=\prod_{v} f_{v}^{G}$ sera $\prod_{v} \varphi_{f_{v}}$. Dans cette section nous nous occupons surtout des fonctions locales de sorte que nous sommes libres de supprimer l'indice $v$.

Les fonctions locales seront 0 aux points singuliers de $\mathfrak{A}=\mathfrak{A}_{v}$, donc en \pm 2 . N'importe quel autre point $b$ de $\mathfrak{A}_{v}$ détermine une classe stable de tores locaux. Si $H$ est un tel tore, il y a un $\gamma \in H(F)$ régulier tel que $\gamma \mapsto b$. Nous posons $\varphi(b)=L\left(s, \sigma_{T / G}\right)|\Delta(\gamma)| f^{H}(\gamma)$, où $T$ est le tore qui contient $\gamma$. La présence ici du facteur $\Delta(\gamma)$ peut sembler bizarre. Mais sans lui la fonction $\varphi$ ne serait pas continue et sa transformée de Fourier décroîtrait trop lentement. Il ne serait pas donc possible d'appliquer la formule de Poisson. 
Nous commençons avec un corps $F$ archimédien. Si $F$ est le corps réel, alors il y a deux classes de sous-groupes de Cartan, $T_{\mathrm{sp}}$ déployé et $T_{\mathrm{com}}$ compact. Pour un $f^{G}$ donné les fonctions $f^{T_{\mathrm{sp}}}$ et $f^{T_{\mathrm{com}}}$ sont des fonctions lisses de $\gamma \in T_{\mathrm{sp}}$ et $\gamma \in T_{\mathrm{com}}$ respectivement. La fonction $\varphi$ est lisse sauf aux points $b= \pm 2$, mais pas nécessairement lisse en ces deux points. Soit $b=\mathbf{c}(\gamma)= \pm(2+x)$ de sorte que $|\Delta(\gamma)|=\left|\sqrt{b^{2}-4}\right|=|x|^{1 / 2} \eta(x)$ avec $\eta$ lisse à $x=0$. Si les valeurs propres de $\gamma \in T_{\mathrm{sp}}$ sont $\pm e^{ \pm t}$, alors $x=t^{2}+a_{2} t^{4}+a_{3} t^{6}+\ldots$ Puisque $f^{T_{\mathrm{sp}}}$ est une fonction lisse de $t^{2}, \varphi(b)$ est pour $x>0$ le produit de $|x|^{1 / 2}$ et d'une fonction lisse en $x$ dans un voisinage de $x=0$ dont le terme constant est $f^{T_{\mathrm{sp}}}( \pm 1)$. Pour des raisons semblables, $\varphi(b)$ est pour $x<0$ le produit de $|x|^{1 / 2}$ et une fonction lisse en $x$ dans un voisinage de $x=0$ dont le terme constant est $f^{T_{\text {com }}}( \pm 1)$. Ces deux constantes ne sont pas nécessairement égales. De toutes façons, $\varphi$ n'est pas nécessairement lisse, mais son comportement est néanmoins assez régulier.

Pour le corps des nombres complexes, les valeurs propres sont $e^{ \pm z}, z=u+i v \in \mathbb{C}$ et $|\Delta(\gamma)|=\left|e^{z}-e^{-z}\right| \mathbb{C}$, mais selon nos conventions cette valeur absolue est le carré de la valeur absolue habituelle $\|x\|$. On a encore $b= \pm(2+x), x=z^{2}+a_{2} z^{4}+\ldots$ et $\left|e^{z}-e^{-z}\right|_{\mathbb{C}}=\|x\| \eta(u, v)$, où $\eta$ est une fonction lisse des deux coordonnées, $\operatorname{Re} x$ et $\operatorname{Im} x$, et est égale à 1 en $x=0$. Il s'ensuit que $\varphi(b)$ est aussi le produit de $\|x\|$, et d'une fonction lisse dans un voisinage de $b= \pm 2$ où il est égal à $f^{T_{\mathrm{sp}}}( \pm 1)$.

Pour les corps non archimédiens, il y a quatres classes de conjugaison stable pour les sous-groupes de Cartan et par conséquent, à chacun des points $b= \pm 2$, quatre valeurs possibles de $f^{H}( \pm 1)$ sont en compétition. Dans un voisinage de ce point la tourtière est coupée en quatre morceaux de tailles peut-être inégales, car il faut distinguer les deux tores ramifiés. Si $\gamma \mapsto b$, et si les valeurs propres de $\gamma$ sont $\lambda^{ \pm 1}$ pour le tore déployé, $\lambda=\alpha \pm \sqrt{\epsilon} \beta$ pour le tore non ramifié, et $\lambda=\alpha \pm \sqrt{\tau} \beta$ pour les tores ramifiés alors $b=\lambda+\lambda^{-1}$. Si $b= \pm(2+x)$, alors $|\Delta(\gamma)|$ est encore $|x|^{1 / 2} \eta(x)$ où $\eta$ est égal à 1 dans un voisinage de $x=0$.

\section{Les fonctions sphériques}

3. Quelques calculs pour les fonctions sphériques. Selon l'article [FLN] et selon les observations des paragraphes précédents, les fonctions $\theta_{v}=\theta_{f_{v}}$ et $\varphi_{v}=\varphi_{f_{v}}$, surtout pour certaines fonctions $f_{v}$ dans l'algèbre de Hecke et pour leurs transformées de Fourier, sont centrales dans la formule des traces, d'abord pour $S L(2)$ mais en toute probabilité aussi pour n'importe quel groupe quasi-déployé. Je ne me trouve pas cependant à mon aise avec elles. Pour gagner moi-même quelque facilité pour leur maniement mais aussi pour que les lecteurs se sentent plus confortable avec elles, je décris quelques propriétés simples mais fondamentales de ces fonctions. Il s'agit en partie de répétitions. Il s'agit aussi de calculs élémentaires, mais la clarté qui en résulte justifie cette descente dans le fastidieux.

La base de Steinberg-Hitchin est la ligne droite sur $F$ et l'application $\mathbf{c}: g \mapsto \operatorname{tr} g$, $g \in S L(2)$. Puisqu'il s'agit dans cette section de calculs locaux, nous pouvons supposer que le caractère local $\chi_{v}$ est tel que $\mathcal{O}_{v}$ soit auto-dual et que sa mesure soit 1 . Les éléments singuliers de la base sont \pm 2 . Ce qui reste correspond aux éléments semi-simples et réguliers de $G$. Pour la plupart nous nous intéressons aux éléments réguliers. Ils sont 
répartis dans des éléments déployés, des éléments non ramifiés et des éléments ramifiés. Dans notre imagination, la base (plutôt les voisinages de \pm 1 dans la base) est, comme une tourtière canadienne, coupée en trois portions triangulaires de taille inégale. Si $\lambda$ et $\bar{\lambda}$ sont les valeurs propres de $g$ et $\mathbf{c}(g)=b$, alors $b=\lambda+\lambda^{-1}$ et

$$
\lambda=\frac{b}{2} \pm \frac{\sqrt{b^{2}-4}}{2} .
$$

Nous supposons que la caractéristique du corps $\mathcal{O}_{v} / \mathfrak{p}_{v}$ n'est pas 2 . À une autre occasion il faudra revenir sur ce cas.

Nous nous intéressons d'abord aux points $b \in \mathcal{O}_{v}$, un ensemble compact dans la base de Steinberg-Hitchin de mesure 1. Supposons d'abord que $b \not \equiv \pm 2\left(\bmod \mathfrak{p}_{v}\right)$. Il y a $q-2$ possibilités pour le résidu $\bar{b}=b\left(\bmod \mathfrak{p}_{v}\right)$. De ceux-ci $(q-3) / 2$ donnent des $\lambda \in F^{\times}$, donc sont déployés, et $(q-1) / 2$ donnent des extensions $F(\lambda)$ non ramifiées. Cela donne des mesures $(q-3) / 2 q$ et $(q-1) / 2 q$.

Considérons le cas où $b \equiv 2\left(\bmod \mathfrak{p}_{v}\right)$, le cas $b \equiv-2\left(\bmod \mathfrak{p}_{v}\right)$ étant tout à fait semblable. On multiplie $b$ et $\lambda$ par -1 . Soit $|b-2|=q^{-k}, k>0$, et $\lambda=\alpha+\beta, \alpha=1+\mu$, $\mu=q^{-k}, \beta^{2}=b^{2}-4=4 \mu+\mu^{2}$. Donc le corps $F(\lambda)$ est ramifié si et seulement si $k$ est impair. Si $k$ est pair et si $\mathfrak{p}_{v}=\varpi_{v}$, alors $\mu=\eta \varpi^{k / 2}, \eta \not \equiv 0\left(\bmod \mathfrak{p}_{v}\right)$ et l'extension est déployée si $\eta$ est un carré modulo $\mathfrak{p}_{v}$ et non ramifiée sinon. Pour un $k$ donné, la mesure dans la base de Steinberg-Hitchin des trois possibilités, donc des intersections des trois portions avec la partie entière $b \in \mathcal{O}_{v}$ de la base, est

$$
\begin{aligned}
& k=2 l>0 \text { pair: déployé } \frac{q-1}{2 q} q^{-2 l} ; \text { non-ramifié } \frac{q-1}{2 q} q^{-2 l} . \\
& k=2 l+1>0 \text { impair: ramifié } \frac{q-1}{q} q^{-2 l-1} .
\end{aligned}
$$

Observons que la parité de $k$ est donnée par la classe du tore

Pour confirmer ces calculs vérifions que la somme de ces mesures est 1.

$$
\left\{\begin{aligned}
\frac{q-3}{2 q}+\frac{q-1}{2 q} & =\frac{q-2}{q} \\
\sum_{l=1}^{\infty} \frac{q-1}{2 q} q^{-2 l} & =\frac{1}{2 q} \frac{1-1 / q}{1-1 / q^{2}}=\frac{1}{2 q} \frac{1}{1+1 / q} \\
\sum_{l=0}^{\infty} \frac{q-1}{q} q^{-2 l-1} & =\frac{1}{q^{2}} \frac{1}{1+1 / q}
\end{aligned}\right.
$$

La deuxième ligne apparaît pour les points déployés et pour les points non ramifiés. Il faut en plus tenir compte pour les deuxième et troisième lignes des deux possibilités $b \equiv \pm 2$ $\left(\bmod \mathfrak{p}_{v}\right)$. Cela donne en tout

$$
\frac{q-2}{q}+\frac{2}{q}=1
$$

Pour examiner $\theta_{f_{v}}$ pour un élément $f_{v}$ dans l'algèbre de Hecke nous employons le Lemma 1 de [L2]. Ce lemme a été formulé pour le groupe $G L(2)$ mais puisque nos intégrales 
orbitales sont des intégrales stables nous pouvons l'utiliser pour $G=S L(2)$. Il faut toutefois tenir compte des normalisations des mesures utilisées dans [FLN] et dans [L2]. Celles de ce dernier article sont différentes de celles de [FLN]. La mesure utilisée ici est celle de $[\mathrm{FLN}]$ :

$$
d \bar{g}_{v}=\frac{L\left(1, \sigma_{G}\right)}{L\left(1, \sigma_{T}\right)} \frac{d_{\mathrm{geom}} g_{v}}{d_{\mathrm{geom}} t_{v}} .
$$

Celle de [L2] était définie comme le quotient d'une sur $G\left(F_{v}\right)$ pour laquelle la mesure de $G\left(\mathcal{O}_{v}\right)$ et celle de $T\left(\mathcal{O}_{v}\right)$ étaient 1 . Observons que $L\left(1, \sigma_{G}\right)=1$ pour $G=S L(2)$ et, sous notre hypothèse sur $\chi_{v}$, la mesure de $G\left(\mathcal{O}_{v}\right)$ par rapport à $d_{\text {geom }} g_{v}$ est selon un calcul bien connu et facile $1-1 / q^{2}$. Pour employer le lemme de [L2] il faut donc calculer la mesure de $T\left(\mathcal{O}_{v}\right)$ par rapport à $L\left(1, \sigma_{T}\right) d_{\text {geom }} t_{v}$. Nous avons fixé la forme sur $T$ qui définit la mesure dans la proposition 3.29 de [FLN]. Pour $S L(2)$ elle est $d \lambda / \lambda$. Donc sur les éléments entiers où $|\lambda|_{v}=1$, c'est simplement $d \lambda$. On vérifie facilement que pour les tores déployés ou non ramifiés

$$
\int_{T\left(\mathcal{O}_{v}\right)} d_{\text {geom }} t_{v}=L\left(1, \sigma_{T}\right) .
$$

Donc dans ces deux cas pour convertir les formules de [FLN] en formules valables avec les conventions de cet article il faut les multiplier par $1-1 / q^{2}=\operatorname{mes} G\left(\mathcal{O}_{v}\right)$.

Pour les tores ramifiés il faut toutefois être attentif. Utilisons la notation empruntée de [SS] et écrivons $\lambda=\alpha+\sqrt{\tau} \beta$. Alors $\alpha^{2}-\tau \beta^{2}=1,|\alpha|=1,|\sqrt{\tau}|=q^{-1 / 2}, \alpha d \alpha=\tau \beta d \beta$ et

$$
d \lambda=d \alpha+\sqrt{\tau} d \beta=\frac{\sqrt{\tau}}{\alpha} \lambda d \beta
$$

de sorte que la mesure $d \lambda / \lambda$ est la mesure $q^{-1 / 2} d \beta$. Il y a toutefois deux points supplémentaires auxquels il faut faire attention. D'abord à chaque $\beta$ correspond deux $\alpha$ et, par conséquent, deux $\lambda$, un proche de 1 et un proche de -1 . Cela ajoute un facteur 2 à la mesure de $T\left(\mathcal{O}_{v}\right)$. Soit $G^{\prime}=G L(2)$ et $T^{\prime}$ le centralisateur du tore ramifié dans $G^{\prime}$. Nous avons déjà observé en vérifiant la formule (2.2.5) que $T\left(\mathcal{O}_{v}\right) \backslash G\left(\mathcal{O}_{v}\right)$ n'est que la moitié de $T^{\prime}\left(\mathcal{O}_{v}\right) \backslash G^{\prime}\left(\mathcal{O}_{v}\right)$, car l'ensemble des éléments de norme 1 dans $F(\lambda)$ est d'indice 2 dans $\mathcal{O}_{v}^{\times}$. Le facteur de conversion pour les tores ramifiés est donc $q^{1 / 2}\left(1-1 / q^{2}\right)$.

En dehors de l'ensemble $|b| \leq 1$ il n'est plus convenable de penser à une tourtière découpée car si $|b|>1$ les racines $\lambda=-b / 2 \pm \sqrt{b^{2}-4} / 2$ appartiennent à $F$ et le tore est déployé.

La possibilité la plus importante est le cas où $f_{v}$ est l'élément neutre de l'algèbre de Hecke. Pour l'élément neutre les formules (2.2.10) sont valables dans le domaine $|b| \leq 1$, c : $\gamma \mapsto b$. Évidemment l'intégrale du premier terme de ces formules sur $|b| \leq 1$ donne $1+1 / q$.

Considérons l'intégrale du deuxième terme, qui est évidemment 0 pour le tore déployé. Pour les $b$ tels que $|b \pm 2| \neq 1,|\Delta(\gamma)|=1$ et la contribution est

$$
-\frac{2}{q} \frac{q-1}{2 q}=-\frac{1-1 / q}{q}
$$


En utilisant les formules (3.1), nous calculons la contribution des autres $b$ non ramifiés comme

$$
-2 \sum_{l=1}^{\infty} \frac{q-1}{q^{2}} q^{-3 l}=-2 \frac{1-1 / q}{q} \frac{q^{-3}}{1-q^{-3}}
$$

car $|\Delta(\gamma)|=|\Delta(b)|=q^{-l}$. La contribution ramifiée sera selon (2.2.10) et (3.2) donnée par

$$
-2 \sum_{0}^{\infty}\left(1-1 / q^{2}\right) q^{-3 l-2}=-2 \frac{\left(1-1 / q^{2}\right)}{q^{2}} \frac{1}{1-q^{-3}} .
$$

La somme de (3.4) et (3.5) est le produit

$$
-\frac{2}{q} \frac{1}{1-q^{-3}}\left(\frac{1}{q^{3}}-\frac{1}{q^{4}}+\frac{1}{q}-\frac{1}{q^{3}}\right)=-\frac{2}{q^{2}} .
$$

La somme de cette fraction et (3.3) est

$$
-\frac{1+1 / q}{q}
$$

Si nous ajoutons celle-ci à la contribution $1+1 / q$ du premier terme des formules (2.2.10), nous obtenons $1-1 / q^{2}$, la mesure de $G\left(\mathcal{O}_{v}\right)$. Ce n'est rien que la proposition 5.6 de [FLN] pour $S L(2)$ et l'élément neutre de l'algèbre de Hecke.

Des autres éléments de l'algèbre de Hecke pour $S L(2)$ peuvent être déduites des fonctions $T_{p}^{m}, m$ pair, de [L2], en introduisant $T^{m}(g)=T_{p}^{m}\left(p^{m / 2} g\right), g \in G=S L(2)$. Ces fonctions sont définies pour n'importe quel corps local non archimédien $F$. À cause de la normalisation de mesures utilisée ici, la transformée, dite parfois de Satake, de $T^{m}$ n'est pas simplement, comme dans [L2], la trace de la représentation irréductible de ${ }^{L} G$ de dimension $m+1$ multipliée par $q_{v}^{m / 2}$. Elle est cette trace multipliée par $q_{v}^{m / 2}\left(1-1 / q_{v}^{2}\right)$. Il faut tenir compte de cette modification en calculant $f^{H}$ pour $f=T^{m}$.

Soit encore $\lambda, \lambda^{-1}$ les deux valeurs propres de $g$ et $b=\lambda+\lambda^{-1}$ sa trace. Soit $\mathfrak{p}_{v}=\left(\varpi_{v}\right)$. Pour que l'intégrale orbitale de $T^{m}$ ne soit pas zéro, il faut que $\varpi_{v}^{m / 2} b$ soit intégral, donc que $\varpi_{v}^{m / 2} \lambda$ et $\varpi_{v}^{m / 2} \lambda^{-1}$ soient intégraux. Si cette condition est satisfaite, les calculs qui ont mené à (2.2.10) donnent pour les fonctions $\theta_{m}=\theta_{T_{m}}$ les valeurs

$$
\begin{cases}q_{v}^{m / 2}\left(1+\frac{1}{q_{v}}\right) & \text { si } T \text { est déployé, } \\ q_{v}^{m / 2}\left(1+\frac{1}{q_{v}}\right)-\frac{2 q_{v}^{m / 2}}{q_{v}}|\Delta(\gamma)| & \text { si } T \text { est non ramifié, } \\ q_{v}^{m / 2}\left(1+\frac{1}{q_{v}}\right)-q_{v}^{(m-1) / 2}\left(1+\frac{1}{q_{v}}\right)|\Delta(\gamma)| & \text { si } T \text { est ramifié. }\end{cases}
$$


Nous sommes donc arrivés à un résultat simple mais frappant, qui simplifiera beaucoup les arguments à suivre. La fonction $\theta_{m}$ est une somme de deux fonctions, dont l'une est le produit de la constante $q^{m / 2}(1+1 / q)$ avec la fonction caractéristique de l'ensemble $\left\{b|| b \mid \leq q^{m / 2}\right\}$, et l'autre est 0 sauf si $|b| \leq 1$. Dans cet ensemble elle est donnée par

$$
\begin{cases}0 & \text { si } T \text { est déployé } \\ -\frac{2}{q_{v}^{m / 2}}|\Delta(\gamma)| & \text { si } T \text { est non ramifié } \\ -q_{v}^{(m-1) / 2}\left(1+\frac{1}{q_{v}}\right)|\Delta(\gamma)| & \text { si } T \text { ramifié. }\end{cases}
$$

où $\mathbf{c}: \gamma \mapsto b$. Nous notons cette deuxième fonction $\eta$.

Nous aurons besoin aussi des formules pour les fonctions $\varphi_{m}=\varphi_{T_{m}}$ rattachées aux opérateurs de Hecke. Pour le tore dploy $T_{\mathrm{sp}}$ et pour la fonction $f^{G}=T^{m}$, la fonction $f^{T_{\mathrm{sp}}}$, qui est ce qu'on appelait jadis la transforme de Harish-Chandra mais qui est donne dans le cadre du groupe $L$ par le produit de la constante $q^{m / 2}(1-1 / q)$ et de la trace de la reprsentation de dimension $m+1$ de ${ }^{L} T_{\mathrm{sp}}$ ou, selon les conventions habituelles, la fonction caractristique de l'ensemble $\left\{\lambda \in F^{\times}\left|q^{-m} \leq\right| \lambda \mid \leq q^{m}\right\}$. Pour obtenir $\varphi_{m}$ sur l'image du tore déployé, il faut simplement multiplier par $|\Delta(\gamma)|$, où $\gamma \mapsto b$.

Pour les autres tores nous rappelons que $f^{H}$ est tel que $\operatorname{tr}\left(\pi_{G}^{\text {st }}\left(f^{G}\right)\right)=\theta\left(f^{H}\right)$ si $\pi_{G}^{\text {st }}$ est le $L$-paquet donné par l'image fonctoriale du caractère $\theta$ de $H$. Si $f^{G}$ est un opérateur de Hecke alors $\operatorname{tr}\left(\pi_{G}^{\text {st }}\left(f^{G}\right)\right)=0$ si $\pi_{G}^{\text {st }}$ n'est pas ramifié. Si $H=T_{\text {ram }}$ alors $\pi_{G}^{\text {st }}$ est toujours ramifié et $f^{H}=0$. Si $H=T_{\text {unr }}$ alors $\pi_{G}^{\text {st }}$ est ramifié sauf si $\theta$ est trivial et alors $\pi_{G}^{\text {st }}$ est l'élément $\pi_{\epsilon}$ de la série principale rattaché à l'homomorphisme

$$
x \mapsto\left(\begin{array}{cc}
\operatorname{sgn}_{\epsilon}(x) & 0 \\
0 & 1
\end{array}\right)
$$

de $F^{\times}$dans ${ }^{L} G$. Le caractère $\operatorname{sgn} n_{\epsilon}$ est celui rattaché à l'extension quadratique non ramifiée de $F$. Par conséquent, $f^{H}$ est la fonction constante $\operatorname{tr} \pi_{\epsilon}\left(f^{G}\right)$. Rappelons que, selon la théorie des fonctions sphériques et les calculs préliminaires de [L2],

$$
\operatorname{tr} \pi_{\epsilon}\left(T^{m}\right)=q^{m / 2}\left(1-1 / q^{2}\right)\left((-1)^{m}+(-1)^{m-1}+\cdots+(-1)^{m}\right) .
$$

Puisque $m$ est toujours pair pour le groupe $S L(2)$, la somme est toujours 1 .

Il est utile de formuler un cas particulier explicitement.

Lemme 3.1. Soit $f=T^{0}$, donc le produit de $1-1 / q_{v}^{2}$ et de l'élément neutre de l'algèbre de Hecke. Alors $\varphi_{f}(b)=0$ si b n'est pas dans $\mathcal{O}_{v}$ ou si b est ramifié. Sinon,

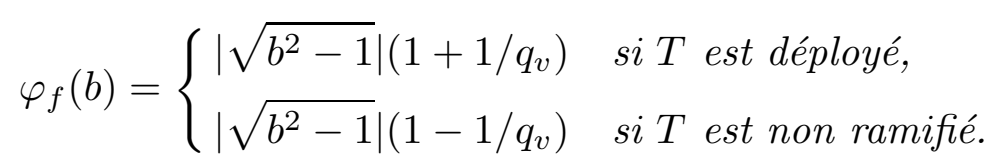

En le vérifiant, il faut rappeler que pour les tores $T=T^{\mathrm{sp}}, T^{\mathrm{unr}}$ la mesure de l'ensemble $\{t \in T|| \lambda \mid=1\}$ est respectivement $1-1 / q_{v}$ et $1+1 / q_{v}$ et que les éléments neutres de 
l'algèbre de Hecke de ces deux groupes sont les fonctions caractéristiques de ces ensembles divisées par leur mesures.

Les formules semblables pour $\varphi_{T^{m}}$ sont

$$
\varphi_{T^{m}}(b)=\left|\sqrt{b^{2}-1}\right|\left(1+1 / q_{v}\right) q_{v}^{m / 2}
$$

si $T$ est dploy et $|b| \leq q^{m / 2}$, mais 0 si $T$ est dploy et $|b|>q^{m / 2}$. Si $T$ est non ramifi mais aussi non dploy,

$$
\varphi_{T^{m}}(b)=\left|\sqrt{b^{2}-1}\right|\left(1-1 / q_{v}\right) q_{v}^{m / 2}
$$

Pour $T$ ramifi, $\varphi_{T_{m}}(b)=0$.

\section{Le comportement asymptotique}

4. Le comportement asymptotique de la transformée de Fourier. Pour comprendre l'admissibilité de la formule de Poisson il y a plusieurs points à expliquer. Quoique nous avons introduit dans [FLN] le paramètre $s$ dans les fonctions $\theta_{v}\left(a_{G}, s\right)$, nous avons observé en discutant la formule (4.8) de cet article-là — une formule à laquelle nous sommes arrivés en utilisant une idée de Jayce Getz — que pour une fonction $f^{G}=\prod_{v} f_{v}^{G}$ globale donnée et pour un ensemble $S^{\prime}$ il est possible de passer à $s=1$. Lorsque nous ajoutons le tore déployé, les expressions (4.12) de [FLN] se comportent mal lorsque $S^{\prime}$ devient de plus en plus grand. Mais, puisque nous les ajoutons à la fois aux deux fonctions $\theta$ et $\varphi$ pour ensuite prendre la différence de ces deux fonctions, tout est légitime. Le danger est que lorsque l'on prend pour la fonction $f^{G}$ le coefficient de $1 / n^{s}$ de la série de Dirichlet (1.14) de [FLN], alors l'ensemble $S^{\prime}$ devient de plus en plus grand et par conséquent les majorations de plus en plus fastidieuses. Ce sont des difficultés que nous avons acceptées et auxquelles nous reviendrons par la suite.

Dans cette section nous examinons, au niveau des produits finis, les conséquences des singularités de $\theta$ et $\varphi$ pour le comportement asymptotique des transformées de Fourier $\hat{\theta}$ et $\hat{\varphi}$ des fonctions locaux $\theta$ et $\varphi$. Si on remplace le caractère de base $\chi=\chi_{v}$ par $\chi^{\prime}, \chi^{\prime}(x)=\chi(a x), a \in F^{\times}$, la transformée de Fourier $\hat{h}$ de n'importe quelle fonction $h$ sur la ligne droite, donc sur la base de Steinberg-Hitchin pour $S L(2)$, est remplacée par $|a|^{1 / 2} h(x a)$. Nous pouvons donc, s'il s'avère opportun, utiliser dans nos calculs locaux une normalisation précise, donc des choix préférés de $\chi$, qui pour les corps non archimédiens seront ceux pour lesquels $\left\{x \mid \chi(x y)=1 \forall y \in \mathcal{O}_{v}\right\}=\mathcal{O}_{v}$.

Nous examinons d'abord le corps des nombres réels.

Lemme 4.1. Soit $h$ une fonction de $x \in \mathbb{R}$ qui pour $x \neq 0$ est le produit de $|x|^{\lambda-1}, \lambda>1$, et d'une fonction $\phi \grave{a}$ support compact, lisse pour pour $x \neq 0$ et telle que les limites

$$
\lim _{x \rightarrow \pm 0} \phi^{(n)}(x), \quad n \geq 0,
$$

de ses dérivées existent. Alors la transformée de Fourier,

$$
\hat{h}(y)=\int_{\mathbb{R}} h(x) e^{i x y} d x,
$$


est $O\left(|y|^{-\lambda}\right)$ pour $y \rightarrow \infty$.

Nous avons choisi $\chi(x)=e^{i x}$, mais si le lemme est valable pour ce choix de caractère $\chi$ il est évidemment valable pour tout choix. Nous avons supposé que $\lambda>1$ car ce cas suffit à nos fins. On aurait pu traiter la condition plus faible $\lambda>0$. Le cas $\lambda=1$ admet une conclusion plus forte car alors on peut exiger que $h$ soit lisse, donc que

$$
\lim _{x \rightarrow-0} \phi^{(n)}(x)=\lim _{x \rightarrow+0} \phi^{(n)}(x)
$$

et alors la fonction $\hat{h}(y)$ est $O\left(|y|^{-\mu}\right)$ pour tout $\mu$. Cela veut dire qu'elle est de décroissance rapide. Le cas $\lambda=0$ est critique car alors la transformée de Fourier n'existe plus.

On a

$$
h^{\prime}(x)=(\lambda-1)|x|^{\lambda-2} \phi(x)+|x|^{\lambda-1} \phi^{\prime}(x)=(\lambda-1)|x|^{\lambda-2} \phi(x)+|x|^{\lambda-2} \phi_{1}(x),
$$

où $\phi_{1}(x)=x \phi^{\prime}(x)$ Puisque la transformée de Fourier de $h^{\prime}(x)$ est $-i y \hat{h}(y)$, il suffit de traiter le cas $1 \leq \lambda \leq 2$. Nous pouvons supposer aussi que $\phi(x)=0$ si $x<0$. Considérons l'intégrale

$$
\int_{0}^{\infty} x^{\lambda-1} \phi(x) e^{i x y} d x
$$

qui est égale à

$$
\left.x^{\lambda-1} \phi(x) \frac{e^{i x y}}{i y}\right|_{0} ^{\infty}-\frac{1}{i y} \int_{0}^{\infty}\left\{(\lambda-1) x^{\lambda-2} \phi(x) e^{i x y}+x^{\lambda-1} \phi^{\prime}(x) e^{i x y}\right\} d x .
$$

Le premier terme est certainement 0 , de sorte qu'il suffit de vérifier que

$$
\int_{0}^{\infty}\left\{(\lambda-1) x^{\lambda-2} \phi(x) e^{i x y}+x^{\lambda-1} \phi^{\prime}(x) e^{i x y}\right\} d x=O\left(|y|^{1-\lambda}\right) .
$$

Le calcul qui a mené de (4.1) à (4.2) s'applique aussi au deuxième terme de cette intégrale. Puisque $\lambda-2>-1$ et $\lambda \leq 2$, il donne une expression qui est $O\left(|y|^{-2}\right)=O\left(|y|^{-\lambda}\right)$. Il suffit donc de montrer que

$$
\int_{0}^{\infty} x^{\lambda-2} \phi(x) e^{i x y} d x=O\left(|y|^{1-\lambda}\right)
$$

Rappelons que $1 \geq \lambda-1>0$ de sorte que l'intégrale converge absolument et est $O(1)$ pour $|y| \rightarrow \infty$.

Certainement si $R=O(1 /|y|)$, nous avons

$$
\int_{0}^{R} x^{\lambda-2} \phi(x) e^{i x y} d x=O\left(|y|^{1-\lambda}\right)
$$


Considérons

$$
\int_{R}^{\infty} x^{\lambda-2} \phi(x) e^{i x y} d x
$$

Une intégration partielle donne

$$
\left.\frac{x^{\lambda-2}}{i y} \phi(x)\right|_{R} ^{\infty}-\frac{1}{i y} \int_{R}^{\infty} x^{\lambda-2} \phi^{\prime}(x) e^{i x y} d x-\frac{\lambda-2}{i y} \int_{R}^{\infty} x^{\lambda-3} \phi(x) e^{i x y} d x .
$$

Le premier terme de cette somme est pour n'importe quel $\lambda$ majoré par $O\left(|y|^{1-\lambda}\right)$. Nous pouvons donc le mettre au rancart. Le deuxième terme est le produit de $i / y$ et d'une intégrale qui est tout à fait pareille à (4.5) sauf qu'une fonction lisse à support compact à été remplacée par une autre. À part une constante $i(\lambda-2)$ le troisème terme est encore (4.5) sauf qu'un facteur $1 / y$ a été ajouté et $\lambda$ remplacé par $\lambda-1$. Après avoir répété cette opération $n$ fois, nous arrivons à une somme finie de termes dont chacun est le produit d'une constante et de

$$
\frac{1}{y^{n}} \int_{R}^{\infty} x^{\lambda-2+k-n} \phi^{(k)}(y) e^{i x y} d x, \quad 0 \leq k \leq n .
$$

Si $k \neq n$, cette expression est certainement $O\left(y^{1-\lambda-k}\right)$, car alors $\lambda-2+k-n<-1$. Si $k=n$, elle est $O\left(1 /|y|^{n}\right)$. Puisque $\lambda-1<1$ il suffit de prendre $n=1$ et le lemme en résulte.

Pour le corps des nombres réels, chacune des fonctions $\theta_{f}$ et $\varphi_{f}$ est la somme d'un nombre fini de translations ou de réflexions de fonctions qui satisfont aux conditions du lemme. Nous pouvons donc appliquer le lemme à leurs transformées de Fourier.

Pour le corps des nombres complexes il y a un lemme semblable. Nous le démontrons avec les mêmes méthodes, que le lecteur peut trouver un peu primitives et sans élégance. Il s'agit, dans les deux lemmes, d'énoncés qui se trouvent sans doute dans toute introduction à la théorie des distributions et ses applications à l'analyse. Lorsque l'heure arrive de passer au cas d'un groupe général, il nous faudra consulter ces livres, mais pour le moment nous nous contentons de méthodes frustes.

Lemme 4.2. Supposons que $h$ soit une fonction de $z \in \mathbb{C}$, de support compact et lisse pour $z \neq 0$. Supposons en plus que dans un voisinage de $z=0, h(z)$ est le produit de $|z|^{\lambda-1}, \lambda>1$, et d'une fonction $\phi$ lisse. Alors la transformée de Fourier de

$$
\hat{h}(w)=\int_{\mathbb{C}} h(z) e^{i \operatorname{Re}(z w)} d x d y, \quad z=x+i y,
$$

est $O\left(|w|^{-\lambda}\right)$.

Rappelons que dans cet article, pour le corps $\mathbb{C},|z|=\|z\|^{2}=x^{2}+y^{2}$.

Nous utilisons encore une récurrence, mais le calcul initial est plus compliqué. La transformée de Fourier

$$
\triangle h=\frac{\partial^{2} h}{\partial x^{2}}+\frac{\partial^{2} h}{\partial y^{2}}
$$


est $-|z| \hat{h}$. Posons $r=\|z\|$ et calculons (4.7). C'est la somme de $\partial^{2} h / \partial x^{2}$ et $\partial^{2} h / \partial y^{2}$, dont les valeurs sont

$(2 \lambda-2)(2 \lambda-3) r^{2 \lambda-4}\left(\frac{\partial r}{\partial x}\right)^{2} \phi+(2 \lambda-2) r^{2 \lambda-3} \frac{\partial^{2} r}{\partial x^{2}} \phi+2(2 \lambda-2) r^{2 \lambda-3} \frac{\partial r}{\partial x} \frac{\partial \phi}{\partial x}+r^{2 \lambda-2} \frac{\partial^{2} \phi}{\partial x^{2}}$

et

$(2 \lambda-2)(2 \lambda-3) r^{2 \lambda-4}\left(\frac{\partial r}{\partial y}\right)^{2} \phi+(2 \lambda-2) r^{2 \lambda-3} \frac{\partial^{2} r}{\partial y^{2}} \phi+2(2 \lambda-2) r^{2 \lambda-3} \frac{\partial r}{\partial y} \frac{\partial \phi}{\partial y}+r^{2 \lambda-2} \frac{\partial^{2} \phi}{\partial y^{2}}$

Rappelons que

$$
\frac{\partial r}{\partial x}=\frac{x}{r}, \quad \frac{\partial r}{\partial y}=\frac{y}{r}
$$

et que

$$
\frac{\partial^{2} r}{\partial x^{2}}=\frac{1}{r}-\frac{x^{2}}{r^{3}}, \quad \frac{\partial^{2} r}{\partial y^{2}}=\frac{1}{r}-\frac{x^{2}}{r^{3}},
$$

de sorte que leur somme est $1 / r$. Nous obtenons pour (4.7) l'expression

$$
(2 \lambda-2)(2 \lambda-3) r^{2 \lambda-4} \phi+(2 \lambda-2) r^{2 \lambda-4} \phi+2(2 \lambda-2) r^{2 \lambda-4}\left(x \frac{\partial \phi}{\partial x}+y \frac{\partial \phi}{\partial y}\right)+r^{2 \lambda-2} \triangle \phi,
$$

qui s'écrit

$$
r^{2 \lambda-4}\left\{(2 \lambda-2)(2 \lambda-3) \phi+(2 \lambda-2) \phi+2(2 \lambda-2)\left(x \frac{\partial \phi}{\partial x}+y \frac{\partial \phi}{\partial y}\right)+\phi_{1}\right\},
$$

où $\phi_{1}=r^{2} \triangle \phi$. Puisque $|z|=r^{2}$, ce calcul permet la même récurrence que pour le corps $\mathbb{R}$. Supposons donc que $1<\lambda \leq 2$.

Pour arriver à l'analogue de (4.2), nous commençons avec

$$
-\|w\| \int r^{2 \lambda-2} \phi(z) e^{i \operatorname{Re}(z w)} d x d y=\int r^{2 \lambda-2} \phi(z) \triangle\left(e^{i \operatorname{Re}(z w)}\right) d x d y .
$$

Nous faisons une intégration partielle double en observant que les dérivées de deuxième order de $r^{2 \lambda-2}$ sont toutes d'ordre au plus $2 \lambda-4>2$ en $r=0$ et par conséquent intégrables. L'analyse exacte doit commencer avec l'intégrale sur la région $r \geq \epsilon>0$, mais les contributions de la frontière disparaissent lorsque $\epsilon \rightarrow 0$. Les intégrations partielles donnent donc

$$
\int \triangle\left(r^{2 \lambda-2} \phi(z)\right) e^{i \operatorname{Re}(z w)} d x d y
$$

et $\triangle\left(r^{2 \lambda-2} \phi(z)\right)$, qui est l'expression (4.7), se calcule en utilisant (4.8). Il est le produit de $r^{2 \lambda-4}$, dont la valeur absolue est intégrable sur des disques $\|z\| \leq R$, l'intégrale étant 
d'ordre $R^{2 \lambda-2}$, et d'une fonction $\phi_{2}$ bornée, lisse et à support compact. Il faut montrer que (4.9) est $O\left(|w|^{1-\lambda}\right)$.

Si $\lambda=2$, l'expression (4.9) est la transformée de Fourier de la fonction $\phi_{1}$. Elle décroît par conséquent rapidement de sorte que l'inégalité voulue est évidente. Supposons donc que $2>\lambda>1$. Puisque $R^{2 \lambda-2}$ est alors $|w|^{1-\lambda}$, nous pouvons, en vérifiant la majoration de (4.9), remplacer le domaine d'intégration par un domaine qui n'exclut qu'un sous-ensemble d'un disque de rayon $R, R \sim 1 /\|w\|$ ou, mieux, un carré de coté $2 R$ dont le centre est à l'origine. Il est en plus permis, pourvu que les majorations établies sont uniformes, de supposer que $w=u$ est réel.

Pour traiter ce qui reste de (4.9), donc l'intégrale sur le domaine $\max \{|x|,|y|\} \geq R$, coupons la région en deux parties,

$$
\begin{aligned}
& D_{1}=\{x+i y|| y \mid>R\} \\
& D_{2}=\{x+i y|| x|>R,| y \mid \leq R\}
\end{aligned}
$$

Nous commençons avec la contribution de (4.10.a),

$$
\int_{|y| \geq R} \int_{-\infty}^{\infty}\left(x^{2}+y^{2}\right)^{\lambda-2} \phi_{2}(x, y) e^{i x u} d x d y
$$

en modifiant l'intégrale intérieure par une intégration partielle pour obtenir,

$$
-\frac{1}{i u} \int_{-\infty}^{\infty}\left(x^{2}+y^{2}\right)^{\lambda-2} \frac{\partial \phi_{2}}{\partial x}(x, y) e^{i x u} d x-\frac{1}{i u} \int_{-\infty}^{\infty} 2 x\left(x^{2}+y^{2}\right)^{\lambda-3} \phi_{2}(x, y) e^{i x u} d x
$$

Puisque la propriété essentielle de $\phi_{2}$ est d'être lisse à support compact, et même à support compact fixe, son remplacement par $\partial \phi_{2} / \partial x$ est sans importance. Ce qui est important, c'est que nous avons, soit ajouté un facteur $u$ au dénominateur, soit ajouté un facteur $u$ au dénominateur et, en même temps, remplacé une fonction homogène du degré total $2 \lambda-4$ en $(x, y)$ par une fonction de degré total $2 \lambda-5$. Ces fonctions sont finies et continues pour $x^{2}+y^{2} \neq 0$. En continuant nous arrivons à une somme finie d'intégrales dont chaque terme est, à un facteur $i^{m}$ près,

$$
\frac{1}{u^{n}} \int_{-\infty}^{\infty} P(x, y)\left(x^{2}+y^{2}\right)^{\lambda-2-n} \phi_{*}(x, y) d x
$$

où $P(x, y)$ est un polynôme homogène de degré $k$ entre $n$ et $2 n$ et $\phi_{*}=\partial^{k} \phi_{2} / \partial x^{k}$. Plutôt que majorer l'intégrale de la valeur absolue de cette fonction sur un domaine $|y| \geq R$, nous majorons

$$
\frac{1}{|u|^{n}} \int_{R_{1}^{2} \geq x^{2}+y^{2} \geq R^{2}}|P(x, y)|\left(x^{2}+y^{2}\right)^{\lambda-2-n} d x d y \leq \frac{C}{|u|^{n}} \int_{R}^{R_{1}} r^{2 \lambda-3+k-2 n} d r .
$$

Ni la valeur de la constante positive $C$ ni la valeur du rayon $R_{1}$, qui est déterminé par le support des fonctions $\phi_{*}$, ne sont importantes. Pour $n$ suffisamment grand, l'intégrale est 
finie et égale à une constante fois $R^{2 \lambda-2+k-2 n}=O\left(|u|^{-2 \lambda+2-k+2 n}\right)$, de sorte que (4.13) est $O\left(|u|^{-2 \lambda+2+(n-k)}\right)=O\left(|u|^{-2 \lambda+2}\right)$.

La contribution de (4.10.b) s'écrit

$$
\int_{|y| \leq R} \int_{|x| \geq R}\left(x^{2}+y^{2}\right)^{\lambda-2} \phi_{2}(x, y) e^{i x u} d x d y .
$$

L'intégrale intérieure est la somme de deux intégrales tout à fait semblables, pour $x \geq R$ et pour $x \leq-R$. Considérons la première. Elle est la somme d'une expression semblable à (4.12.a), à savoir la somme de

$$
-\frac{1}{i u} \int_{R}^{\infty}\left(x^{2}+y^{2}\right)^{\lambda-2} \frac{\partial \phi_{2}}{\partial x}(x, y) e^{i x u} d x-\frac{1}{i u} \int_{R}^{\infty} 2 x\left(x^{2}+y^{2}\right)^{\lambda-3} \phi_{2}(x, y) e^{i x u} d x
$$

et de

$$
-\frac{1}{i u}\left(R^{2}+y^{2}\right)^{\lambda-2} \phi_{2}(R, y) e^{i R u} .
$$

L'intégrale sur $-R \leq y \leq R$ de la valeur absolue de cette dernière expression est $O\left(R^{2 \lambda-2}\right)$. Une fois que cette intégrale et une intégrale semblable pour l'intervalle $(-\infty,-R)$ ont été majorées, le traitement de (4.10.b) est pareil à celui de (4.10.a).

Observons qu'il n'était pas absolument nécessaire de ramener les deux lemmes au cas où $2 \geq \lambda>1$, mais la récurrence aussi bien que la resemblance des deux démonstrations sont réconfortantes.

Pour un corps local archimédien les fonctions $\theta$ et $\varphi$ sont encore de support compact et lisse sauf aux points $b= \pm 2$. Les singularités de $\theta$ aux points $b= \pm 2$ sont décrites dans le lemme 2.2.2. Dans un voisinage de ces deux points, $\theta$ est la somme d'une fonction lisse à support compact, donc la somme d'une fonction dont la transformée de Fourier est aussi lisse et à support compact et d'une deuxième fonction, qui est essentiellement $|\Delta|$. Plus précisement, elle est la somme de quatre fonctions, dont chacune est supportée par l'intersection d'un domaine $|b \pm 2| \leq \epsilon, \epsilon>0$, avec l'image d'une des quatre classes de sous-groupes de Cartan. Sur ce support elle est le produit d'une constante avec $|\Delta|$. À une facteur uniforme près, ces constantes sont données par les formules (2.2.4). Les fonctions $\varphi$ admettent une description pareille, sauf que la première des deux fonctions est 0 . Par contre, les quatre constantes qui interviennent dans la description de la deuxième fonction sont largement indépendantes, puisque les fonctions $f^{H}$ pour les quatres tores le sont. Nous avons donc besoin du lemme suivant, dans lequel nous supposons encore que la caractéristique du corps $\mathbb{F}_{v}$ est impaire. Si $b= \pm 2+x,|x|<1$, alors $|\Delta(b)|=\left|\sqrt{b^{2}-4}\right|=$ $\left|x^{1 / 2}\right|$. En plus, si $\gamma \mapsto b$, la classe de conjugaison stable du sous-groupe de Cartan qui contient $b$ est déterminée par $x$ modulo $\left(F^{\times}\right)^{2}$. Si $\left|b^{2}-4\right|=1$, cette classe de conjugaison est détérminée par la classe quadratique de $b^{2}-4$ modulo $\mathfrak{p}$.

Lemme 4.3. Soit $H$ un sous-groupe de Cartan de $G$. Soit $h$ une fonction de $x \in F$ qui est le produit de $|x|^{\lambda-1}, \lambda>1$, et d'une fonction $\phi$ lisse dont le support est l'intersection 
d'un ensemble compact de $F$ ouvert avec une classe dans $F^{\times} / F^{\times^{2}}$. Alors la transformée de Fourier,

$$
\hat{h}(y)=\int_{\mathbb{R}} h(x) \chi(x y) d x
$$

est $O\left(|y|^{-\lambda}\right)$ pour $|y| \rightarrow \infty$.

Puisque $|x|$ est localement constant en dehors de $\{0\}$, nous pouvons suppposer que $\phi$ est constant sur son support. Puisque la transformée de Fourier de $x \mapsto h(a x)$ est $\hat{h}(y / a) /|a|$, nous pouvons supposer que le support de $h$ est l'ensemble $\left(F^{\times}\right)^{2} \cap \mathcal{O}$. Nous pouvons supposer aussi que le conducteur de $\chi$ est $\{x|| x \mid \leq 1\}$ et que la mesure de $\mathcal{O}$ est égale à 1. Alors

$$
\int_{|x| \leq q^{m}} \chi(x y) d x=\left\{\begin{array}{lll}
0 & \text { si }|y|>q^{-m} \\
q^{m} & \text { si }|y| \leq q^{-m}
\end{array}\right.
$$

Par conséquent,

$$
\int_{|x|=q^{m}} \chi(x y) d x= \begin{cases}0 & \text { si }|y|>q^{-m+1} \\ -q^{m-1} & \text { si }|y|=q^{-m+1} \\ q^{m}-q^{m-1} & \text { si }|y|<q^{-m+1}\end{cases}
$$

Nous avons toutefois besoin d'informations plus précises. Si $x \in\left(F^{\times}\right)^{2}$ alors $|x|=q^{m}$, avec un exposant pair. Si $m$ est pair et $|x|=q^{m}$, alors $x \in\left(F^{\times}\right)^{2}$ si et seulement si $x$ est congruent à un carré modulo $\mathfrak{p}^{m-1}$. La mesure de $\left\{x \in\left(F^{\times}\right)^{2}|| x \mid=q^{m}\right\}$ est par conséquent $(1-1 / q) q^{m} / 2$ de sorte qu'il faut modifier légèrement ces formules:

$$
\int_{\substack{|x|=q^{m} \\ x \in\left(F^{\times}\right)^{2}}} \chi(x y) d x= \begin{cases}0 & \text { si }|y|>q^{-m+1} \\ \alpha q^{m}\left(1-q^{-1}\right) & \text { si }|y|=q^{-m+1} \\ \left(q^{m}-q^{m-1}\right) / 2 & \text { si }|y|<q^{-m+1}\end{cases}
$$

oú $\alpha=\alpha\left(y / z^{2}\right)$ est une somme de type gaussien,

$$
\alpha\left(y / z^{2}\right)=\frac{1}{q} \sum_{\substack{x \in \mathbb{F}_{q}^{2} \\ x \neq 0}} \chi\left(x y / z^{2}\right), \quad|z|=q^{-m / 2}
$$

et $x \mapsto \chi\left(x y / z^{2}\right)$ est traité comme un caractère $\tilde{\chi}$ du groupe multiplicatif $\mathbb{F}_{q}^{\times}$. Il s'ensuit que

$$
\hat{h}(y)=\frac{1}{2} \sum_{0}^{\infty} q^{2(1-\lambda) m}\left(q^{-2 m}-q^{-2 m-1}\right)=\frac{1}{2} \frac{1-1 / q}{1-1 / q^{2 \lambda}}
$$


si $|y| \leq 1$. Si $|y|=q^{n}$, où $n>0$ est impair, alors

$$
\hat{h}(y)=q^{(1-\lambda)(n-1)}\left(\alpha q^{-n+1}-q^{-n}\right)+\frac{1}{2} \sum_{m=(n+1) / 2}^{\infty} q^{2 m(1-\lambda)}\left(q^{-2 m}-q^{-2 m-1}\right)
$$

qui est égal à

$$
\frac{\alpha}{q^{(n-1) \lambda}}-\frac{1}{q^{(n-1) \lambda+1}}+\frac{1}{2 q^{(n+1) \lambda}} \frac{1-1 / q}{1-1 / q^{2 \lambda}}
$$

mais si $n>0$ est pair,

$$
\hat{h}(y)=\frac{1}{2} \sum_{m=n / 2}^{\infty} q^{2(1-\lambda) m}\left(q^{-2 m}-q^{-2 m-1}\right)=\frac{1}{2 q^{n \lambda}} \frac{1-1 / q}{1-1 / q^{2 \lambda}}
$$

Observons que la constante implicite dans le majorant du lemme n'est pas uniforme en $q$.

Ces trois lemmes nous permettront d'utiliser un quatrième lemme.

Lemma 4.4. Soit $h=\prod_{v \in S^{\prime}} h_{v}$. S'il existe des constantes $c>0, d>0$, telles que $\left|h_{v}(a)\right| \leq c \min \left\{1,|a|_{v}^{-1-d}\right\}$ pour tout $a \in F_{v}$ et tout $v \in S^{\prime}$, alors

$$
\sum_{a \in F_{S^{\prime}}}|h(a)|<\infty
$$

Pour la démonstration nous pouvons supposer que pour chaque $v \in S^{\prime}, h_{v}(a)=$ $\min \left\{1,|a|_{v}^{-1-d}\right\}$. Choisissons $\epsilon<1 / 2$ et posons

$$
X=\left\{\left\{x_{v} \mid v \in S^{\prime}\right\}|| x_{v} \mid<\epsilon \forall v\right\} .
$$

L'intersection de $\alpha+X$ avec $\beta+X, \alpha \in F_{S^{\prime}}, \beta \in F_{S^{\prime}}$, avec $\alpha \neq \beta$, est vide. Soit $\mu$ la mesure de $X$. Soit $\delta_{1}=(2 / 3)^{-1-d}, \delta_{2}=2^{-1-d}$. Supposons que $a, b \in F_{v}$ et que $|b|_{v}<\epsilon$.

Si $|a|_{v} \geq 1$ alors $\min \left\{1,|a|_{v}^{-1-d}\right\}=|a|_{v}^{-1-d}$ et $3|a|_{v} / 2 \geq|a+b|_{v} \geq|a|_{v} / 2$ de sorte que

$$
\delta_{2}^{-1}|a|_{v}^{-1-d} \geq|a+b|_{v}^{-1-d} \geq \delta_{1}^{-1}|a|_{v}^{-1-d} .
$$

Par conséquent,

$$
\int_{a+X} \min \left\{\delta_{2},|a+b|_{v}^{-1-d}\right\} d b \geq \frac{\mu}{\delta_{1}}|a|_{v}^{-1-d}
$$

Si $|a|_{v} \leq 1$, alors $|a+b|_{v} \leq 2$ et $|a+b|_{v}^{-1-d} \geq \delta_{2}$, de sorte que $\min \left\{\delta_{2},|a+b|_{v}^{-1-d}\right\}=\delta_{2}=$ $\delta_{2} \min \left\{1,|a|_{v}^{-1-d}\right\}$. Il en résulte pour ce cas que

$$
\int_{a+X} \min \left\{\delta_{2},|a+b|_{v}^{-1-d}\right\} d b=\mu \delta_{2} \min \left\{1,\left|a_{v}\right|^{-1-d}\right\}
$$


Puisque $\delta_{2}<1<\delta_{1}$, la somme (4.18) est majorée par

$$
\left(\frac{\delta_{1}}{\mu \delta_{2}}\right)^{\left|S^{\prime}\right|} \prod_{v \in S^{\prime}} \int_{F_{v}} \min \left\{\delta_{2},|b|_{v}^{-d-1}\right\} d b .
$$

Chacune des intégrales dans le produit est finie. Le lemme s'ensuit.

Nous aurons besoin plus tard de quelques propriétés élémentaires de la fonction $\alpha(y)$ sur l'ensemble des éléments de $\mathfrak{p}^{-1}-\mathfrak{p}$. Elle ne dépend que de $y$ modulo $\mathfrak{p}$. Considérons, pour deux éléments $a$ et $b$ non nuls du corps résiduel, le nombre $(q-1) n(a, b)$ de solutions de l'équation $x^{2}=a y^{2}+b z^{2}, a b \neq 0, x y z \neq 0$. Les nombres $n(a, b)$ sont donnés dans deux matrices, selon le cas que -1 est un carré dans le corps ou non.

$$
\begin{aligned}
& b=+\quad b=- \\
& \begin{array}{r}
\quad b=+\quad b=- \\
-1=-. \quad a=+\quad q-3
\end{array} \\
& \begin{array}{rlll}
-1=+: & a=+ & q-5 & q-1 \\
& a=- & q-1 & q-1
\end{array} \\
& -1=- \text { : } \\
& \begin{array}{lll}
a=+ & q-3 & q-3 \\
a=- & q-3 & q-3
\end{array}
\end{aligned}
$$

Les coefficients des deux matrices, qui donnent les valeurs de $n(a, b)$, dépendent du caractère quadratique dans $\mathbb{F}_{v}$ des trois valeurs $-1, a, b$, que nous donnons non pas avec des symboles de Legendre mais en écrivant $-1= \pm, a= \pm, b= \pm$. L'indice des lignes est $a$, celui des colonnes est $b$. Les matrices sont symétriques. Quoiqu'il s'agit des calculs familiers, nous vérifions ces valeurs. Si $a=+$ il y a $(q-1)(q-3)$ valeurs non nulles de $x^{2}-a^{2} y^{2}, x y \neq 0$; si $a=-$, il en y a $(q-1)^{2}$. Par conséquent, la somme des lignes ou des colonnes des deux matrices est selon le cas $2(q-3)$ ou $2(q-1)$ car il y a $q-1$ choix de $z$ et $(q-1) / 2$ éléments $b \neq 0$ qui sont égaux à un carré. Si $a=+$, donc $a=d^{2}$ et si $x^{2}-a y^{2}=b$ alors $b=u v, u=x-d y, v=x+d y$. Si $b=+$ et $x y \neq 0$, cela donne $q-5$ choix pour $(u, v)$ si $-1=+$ et $q-3$ si $-1=-$. Si $b=-$ et $x y \neq 0$ cela donne $q-1$ choix pour $-1=+$ et $q-3$ pour $-1=-$.

Il y a deux valeurs possibles pour la somme de (4.16) selon le choix de $y$. Si elles sont $U$ et $V$ leur somme est -1 . Leur produit

$$
U V=\sum_{\substack{x, y \in \mathbb{F}_{q}^{2} \\ x y \neq 0}} \tilde{\chi}(x+a y), \quad a=-,
$$

dépend du caractère quadratique de -1 . Si $-1=+$ alors $x+$ ay n'est jamais congru à 0 . Il en résulte que $U V=-(q-1) / 4$ de sorte que $U, V=-1 / 2 \pm \sqrt{q} / 2$. Si $-1=-$ il résulte de $(4.20)$ que $U V=(q+1) / 4$ de sorte que $U, V=-1 / 2 \pm \sqrt{-q} / 2$.

\section{La formule de Poisson}

5. La formule de Poisson. ${ }^{1}$ Rappelons d'abord les simplifications introduites dans la section $\S 4$ de $[\mathrm{FLN}]$. Nous avons d'abord remplacé le facteur $L_{S^{\prime}}\left(s, \sigma_{T / G}\right)$ par 1 . Cela

\footnotetext{
${ }^{1}$ Je souligne tout de suite deux composantes décisives de l'argument de cette section sur lesquelles il est facile à se méprendre. Pour passer à la formule de Poisson il faut ajouter les éléments déployés. Sinon
} 
introduit dans ce facteur une erreur $\operatorname{Err}_{1}=o(s-1)$, qui est petite pourvu que $s-1>0$ soit assez petit et qui ne dépend pas de $f^{G}$, mais cette approximation exige des choix particuliers de l'ensemble $S^{\prime}$, donc, par exemple, que $S^{\prime}=\left\{v \mid q_{v} \leq n\right\}$. Nous supposons par la suite que $S^{\prime}$ est de cette forme. Il y avait aussi un facteur $\Pi_{S^{\prime}}=1+\operatorname{Err}_{2}$ mais $\operatorname{Err}_{2}=o(s-1)$ sans autre condition sur $S^{\prime}$ autre qu'il soit assez grand. En utilisant ces deux approximations dans lesquelles la première des deux erreurs dépend de $f^{G}$ mais non pas la deuxième, nous sommes passés à une somme pour la fonction

$$
\prod_{v \in S^{\prime}} \theta_{v}\left(a_{v} ; s\right), \quad t_{v} \mapsto a_{v}
$$

où $a_{v}$ est dans la base de Steinberg-Hitchin locale. La première erreur dépend de $f^{G}$ car elle est une erreur qui dépend des représentations $\sigma_{T / G}$, dont il n'y a qu'un nombre fini parce que pour une fonction $f^{G}$ donnée il n'y a qu'un nombre fini de classes stables régulières pour lesquelles les intégrales orbitales sont différentes de 0 .

Ensuite, en posant $s=1$ et avec une petite erreur $\operatorname{Err}_{3}$ qui dépend maintenant des fonctions $f_{v}^{G}$ et de $S^{\prime}$, nous arrivons à une somme

$$
\sum_{b \in \mathfrak{B}_{S^{\prime}}}^{\prime} \theta_{S^{\prime}}(b)=\sum_{b \in \mathfrak{B}_{S^{\prime}}}^{\prime} \prod_{v \in S^{\prime}} \theta_{v}(b), \quad \theta_{v}(b)=\theta_{v}(b ; 1)
$$

dans laquelle on exige d'abord — c'est la signification du symbole " $\sum$ prime" — que le tore défini par $b \in \mathfrak{B}_{S^{\prime}}$, supposé régulier, ne contient pas de sous-tore déployé. Puisqu'il s'agit du groupe $G=S L(2)$, pour lequel $\mathfrak{B}_{S^{\prime}}=F_{S^{\prime}}$, cela veut dire que nous excluons pour le moment les $b$ déployés, aussi bien que $b= \pm 2$, mais ces deux derniers points ne donne qu'une somme finie. Il faudra par contre nous occuper de l'absence des $b$ déployés.

L'analyse sera donc délicate et fastidieuse et je ne l'aborde pas sérieusement dans cet article. Je veux néanmoins introduire ce qui je crois sera l'outil principal. Une fois que nous avons passé à l'ensemble $S^{\prime}$ fini de sorte que nous pouvons poser $s=1$, il nous faudra ensuite passer avec toute l'attention nécessaire à la limite où $S^{\prime}$ englobe l'ensemble de toutes les places du corps global $F$. En particulier, même pour $G=S L(2)$, ce n'est qu'en passant à cette limite que la proposition 5.6 de [FLN], qui donne la contribution de la représentation triviale, devient utile.

Pour arriver à cette contribution il faut toutefois employer la formule de Poisson, qui s'ajoute alors à la formule des traces. Cependant, nous ne sommes pas encore en état de faire car il nous manque les $b$ déployés, $b=\lambda+\lambda^{-1}$, avec $\lambda \in F^{\times}$. Pour des groupes autres

la somme de Poisson est incomplète. Mais alors le passage à la limite $S^{\prime} \rightarrow \infty$ mène à des difficultés. Pour les surmonter on soustrait les contributions des représentations automorphes de type dièdre, même pour le tore déployé. L'infini potentiel qui apparaît lorsque $S^{\prime} \rightarrow \infty$ est alors présent deux fois avec des signes différent de façon qu'il est légitime de passer à la limite. Ces contributions supplémentaires s'expriment à partir des fonctions $\varphi$. Les fonctions locales $\theta_{v}$ et $\varphi_{v}$ ont des singularités qui ne s'annulent pas! Mais elles sont suffisamment lisses que nous pouvons, au niveau local, utiliser la somme de Poisson pour chacune. Cela cause, comme nous expliquons dans $\S 7$, des difficultés lorsque $S^{\prime} \rightarrow \infty$ qui ne sont à présent que partiellement résolues et dont l'étude n'est pas abordée dans cet article. 
que $S L(2)$ et $G L(2)$ les problèmes seraient même plus graves car il manquerait tous les éléments partiellement déployés. Au niveau d'un $S^{\prime}$ fini, il n'y a pas toutefois de problème car si $T$ est le tore déployé le produit

$$
\prod_{v \in S^{\prime}} L\left(1, \sigma_{T / G}\right)=\prod_{v \in S^{\prime}} \frac{1}{1-1 / q_{v}}
$$

est fini et la fonction $\prod_{v \in S^{\prime}} \theta_{v}\left(b_{v}\right), \theta_{v}=\theta_{f_{v}}$ définie même si $b_{v} \in T\left(F_{v}\right)$. Donc au niveau fini il n'y pas de problème avec la somme de Poisson

$$
\sum_{b \in F_{S^{\prime}}} \theta(b), \quad \text { où } \quad \theta(b)=\prod_{v \in S^{\prime}} \theta_{v}(b) .
$$

Grâce au lemme 4.4, même la formule de Poisson

$$
\sum_{b \in F_{S^{\prime}}} \theta(b)=\sum_{b \in F_{S^{\prime}}} \hat{\theta}(b)
$$

est valable, car $F_{S^{\prime}} \backslash \mathbb{A}_{S^{\prime}}$ est compact et sa mesure est 1. La difficulté, c'est qu'avec un $S^{\prime}$ qui s'agrandit, le produit (5.2) va vers l'infini. Il n'est donc encore pas question d'une convergence possible. Pour se conformer à la notation de [FLN], il est mieux de commencer avec l'ensemble $S^{\prime}$ donné par l'observation de Getz et de le remplacer par des ensembles $S^{\prime \prime}$ de plus en plus grands. Il faut alors ne pas oublier que $F_{S^{\prime \prime}}$ est bien plus grand que $F_{S^{\prime}}$ et que les fonctions $\theta_{v}, v \in S^{\prime \prime}-S^{\prime}$, ne sont pas les fonctions caractéristiques de $\mathcal{O}_{v}$ de sorte que les deux sommes,

$$
\sum_{b \in F_{S^{\prime}}} \hat{\theta}_{S^{\prime}}(b), \quad \sum_{b \in F_{S^{\prime \prime}}} \hat{\theta}_{S^{\prime \prime}}(b)
$$

sont très différentes même si dans la somme

$$
\sum_{b \in F_{S^{\prime \prime}}} \theta_{S^{\prime \prime}}(b)
$$

les termes $\theta_{S^{\prime \prime}}(b)$, avec $b \in F_{S^{\prime \prime}}-F_{S^{\prime}}$, sont 0 et $\theta_{S^{\prime \prime}}(b)=\theta_{S^{\prime}}(b)$, pour $b \in F_{S^{\prime}}$, de sorte que les deux sommes de (5.3) ont la même valeur.

Que le produit (5.2) devienne de plus en plus grand lorsque $S^{\prime \prime} \rightarrow \infty$ reste un problème grave. Il y a néanmoins une possibilité de s'en tirer, car on a le lemme suivant qui est une conséquence des définitions.

Lemme 5.1. Si $b \in F_{v}$ est déployé, donc, en particulier, l'image $\mathbf{c}(\gamma)$ de $\gamma$ dans $G\left(F_{v}\right)$, alors

$$
\varphi_{f_{v}}(b)=|\Delta(b)|{ }_{v} \theta_{f_{v}}(b) .
$$


Nous écrivons plutôt $\varphi_{v}$ et $\theta_{v}$. La fonction $\varphi=\varphi_{S}^{\prime}$ sur $\mathbb{A}_{S^{\prime}}$ est le produit $\prod_{v \in S^{\prime}} \varphi_{v}$. Encore grâce au lemme 4.4 la formule de Poisson est valable pour $\varphi$ et $\hat{\varphi}$ et pour la différence, $\theta-\varphi$. Pour la différence, les termes $\theta(b)-\varphi(b)=0$ si $b \in F^{\times}$est déployé, car $S^{\prime}$ a été choisi tel que $|\Delta(b)|_{v}=1$ si $\theta_{S^{\prime}}(b) \neq 0$. On aura donc $\theta_{S^{\prime \prime}}-\varphi_{S^{\prime \prime}}=0$ pour tout $S^{\prime \prime} \supset S^{\prime}$. Par conséquent, le manque de convergence des deux termes, $\theta(b)$ et $\varphi(b)$, dont nous prenons la différence n'est pas gênant. Ils s'annulent, l'un l'autre. Nous pourrions aussi éliminer les termes déployés en prenant à une seule place une fonction $f_{v}$ donnée comme coefficient matriciel d'une représentation supercuspidale. Mais cela me semble une astuce factice. Je crois plutôt que le lemme 5.1 soit la clé de la solution des problèmes graves de convergence rencontrés au passage à la limite des ensembles $S^{\prime \prime}$ de plus en plus grands.

Heureusement la différence

$$
\sum_{b \in F}(\theta(b)-\varphi(b))
$$

a une interprétation simple. Les termes où $b$ est déployé sont 0 . Il reste à interpréter la somme

$$
\sum_{b \text { elliptique }} \varphi(b)
$$

Nous sommes arrivés à la somme

$$
\sum_{b \text { elliptique }} \theta(b)
$$

en commençant avec la somme

$$
\sum_{\pi^{\mathrm{st}}} \operatorname{tr} \pi^{\mathrm{st}}(f)
$$

où $f=f^{G}=\prod f_{v}$ était le produit de fonctions lisses à support compact, et en utilisant la formule des traces. Avec le même $f$, considérons

$$
\sum_{H \text { elliptique }} \sum_{\theta_{H} \neq 1} \operatorname{tr}\left(\theta_{G}^{\text {st }}(f)\right)=\sum_{H \text { elliptique }} \sum_{\theta_{H} \neq 1} \operatorname{tr}\left(\theta_{H}\left(f^{H}\right)\right) .
$$

Les $\theta_{H}$ parcourent l'ensemble de caractères non triviaux de $H(F) \backslash H\left(\mathbb{A}_{F}\right)$ et $\theta_{G}$ est l'image par fonctorialité de $\theta_{H}$. Si on fixe une fois pour toutes un ensemble $S_{0}$ en dehors duquel $f$ soit un élément de l'algèbre de Hecke, alors l'ensemble $\mathfrak{H}$ des $H$ qui interviennent est fini, car $f_{v}^{H}=0$ si $f_{v}$ est dans l'algèbre de Hecke et $H$ est ramifié en $v$. Nous pouvons par conséquent ne prendre la somme que sur les $\mathfrak{H}$ sans ramification en dehors de $S_{0}$ et pour chaque $H \in \mathfrak{H}$ ajouter $\theta_{H}$ trivial. Nous pouvons supposer que $S_{0} \subset S^{\prime}$.

Pour un tore elliptique $H$, le quotient $H(F) \backslash H\left(\mathbb{A}_{F}\right)$ est compact de sorte que nous pouvons utiliser la formule des traces pour $H$. Elle donne

$$
\sum_{\theta_{H}} \operatorname{tr}\left(\theta_{H}\left(f^{H}\right)\right)=\operatorname{mes}\left(H(F) \backslash H\left(\mathbb{A}_{F}\right)\right) \sum_{\gamma \in H(F)} f^{H}(\gamma) .
$$


Nous pouvons écarter pour le moment $\gamma= \pm 1$ pour n'y revenir que bien plus tard, certainement pas dans cet article, et pour les autres, ajouter le facteur $|\Delta(\gamma)|=\prod_{v}|\Delta(\gamma)| v=1$. La mesure est donnée comme dans $[\mathrm{FLN}]$ par la formule de Ono. Elle est $L\left(1, \sigma_{T / G}\right)$. Par conséquent le côté droit de (5.8) peut s'écrire

$$
\sum_{\gamma} L\left(1, \sigma_{T / G}\right)|\Delta(\gamma)| f^{H}(\gamma)
$$

Pour une fonction $f$ donnée et pour chaque $H \in \mathfrak{H}$ il n'y a qu'un nombre fini d'éléments $\gamma \in H(F)$ tels que $f^{H}(\gamma) \neq 0$. Nous pouvons donc supposer que pour tous ces $H$ et tous ces $\gamma,|\Delta(\gamma)|_{v}=1$ si $v \notin S^{\prime}$. Nous avons calculé les fonctions $f_{v}^{H}$ pour une fonction $f_{v}$ dans l'algèbre de Hecke à la fin de la section $\S 3$. Puisque $f_{v}^{H}$ est presque partout la fonction caractéristique de $\left\{\gamma \in H\left(F_{v}\right)|| \lambda \mid=1\right\}$, nous pouvons supposer que $f_{v}^{H}(\gamma)=1$ si $v \notin S^{\prime}$. Encore une fois nous pouvons approximer $L\left(1, \sigma_{T / G}\right)$ par $\prod_{v \in S^{\prime}} L_{v}\left(1, \sigma_{T / G}\right)$, $S^{\prime} \supset S_{0}$. Nous avons par conséquent réussi, au prix de quelques erreurs qui deviennent de plus en plus petites lorsque $S^{\prime}$ grandit, à remplacer (5.9) par

$$
\sum_{\gamma \in H(F)} \varphi(b), \quad \gamma \mapsto b \in F=\mathfrak{B}
$$

où $\varphi(\gamma)=\prod_{v \in S^{\prime}} \varphi_{v}(\gamma)$. Implicite dans cette somme est la condition que $\gamma$ et forcément aussi $b=\operatorname{tr} \gamma$ sont des entiers en dehors de $S$, donc que $b \in F_{S^{\prime}}$.

Pour arriver à (5.5) nous pouvons ajouter aux sommes (5.6) et (5.10) pour $\theta$ et $\varphi$ les contributions des tores déployés. Plus précisément, on commence avec la somme sur $H \in \mathfrak{H}$ des sommes $(5.8)$ et on ajoute non seulement les tores elliptiques en dehors de $\mathfrak{H}$ qui contribuent 0 mais aussi le tore déployé. Cela donne à part les contributions des $\gamma$ singuliers, à part les contributions des points du domaine fondamental et à part, peut-être, de quelques points spéciaux du spectre continu de $L^{2}\left(G(F) \backslash G\left(\mathbb{A}_{F}\right)\right.$ à la formule des traces, donc en somme à part des contributions dont on peut sans trop de difficultés rendre des comptes,

$$
\sum_{\pi^{\mathrm{st}}} m_{\pi}^{\mathrm{st}} \operatorname{tr} \pi^{\mathrm{st}}(f)-\sum_{H \text { elliptique }} \sum_{\theta_{H}} \operatorname{tr} \theta_{G}^{s t}(f)
$$

Il faut s'attendre à ce qu'après un examen soigneux de la formule des traces avec tous les termes supplémentaires, on trouvera des contributions qui annulent les contributions non voulues du deuxième, donc les images fonctorielles des représentations triviales des $H\left(\mathbb{A}_{F}\right)$.

Dans mes explications j'ai esquivé bon nombre de difficultés mineures car avant que les difficultés majeures ne soient entamées cela ne vaut guère la peine d'y donner trop de temps. Une fois la formule de Poisson utilisée pour remplacer (5.5) par

$$
\sum_{b \in F}(\hat{\theta}(b)-\hat{\varphi}(b)),
$$


ce qui est maintenant possible, on prend pour $f$ les coefficients $f_{n}$ de l'expansion

$$
f(s)=\sum_{n=0}^{\infty} \frac{f_{n}}{n^{s}}
$$

de la fonction (1.14) de [FLN]. La difficulté analytique centrale sera, à mon avis, de traiter le comportement asymptotique de (5.12) pour ces fonctions. Il ne s'agit pas d'un problème qui, même pour $G=S L(2)$, cédera immédiatement à nos tentatives de le résoudre.

J'observe que les fonctions $\varphi_{v}$ n'ont pas été définies si le corps résiduel de $F_{v}$ est de caractéristique 2. Cela cause certainement des ennuis. Le lecteur prudent peut simplement supposer que si $f=\prod_{v} f_{v}$, alors à toute place où la caractéristique résiduelle est 2 la fonction $f_{v}$ est dans l'algèbre de Hecke. Il pourrait aussi examiner de plus près la théorie des représentations de $S L(2)$ sur un corps local de caractéristique résiduelle 2.

\section{Les fonctions $L$}

6. Les fonctions $L$. Nous avons défini dans [FLN] les fonctions $L$ locales non ramifiées à partir des opérateurs locaux de la formule (1.11) de cet article. Pour pouvoir poursuivre nos réflexions sur le comportement asymptotique nous explicitons cette formule pour une représentation $\rho$ du groupe ${ }^{L} G$ dans la forme $P G L(2)$, donc sans le facteur galoisien de $S L(2) \times \operatorname{Gal}(K / F)$ qui est en principe possible. Il s'agit des propriétés combinatoires des représentations du groupe $P G L(2)$, mais il est plus agréable de travailler avec celles de $S L(2)$, qui sont plus nombreuses, contenant une représentation irréductible de tout degré, pair ou impair. Il est même mieux d'utiliser $G L(2)$, où $\rho_{m}$ sera la représentation sur les tenseurs symétriques de degré $m$, et $T_{k}^{m}$ la fonction $T_{k}^{m}(g)=T_{p}^{m}\left(\varpi^{-k} g\right)$. À gauche, la fonction est celle de [L2], où la notation n'est pas celle de cet article. Dans cet article, $T_{k}^{0}$ sera la fonction caractéristique de l'ensemble de matrices scalaires

$$
\left(\begin{array}{cc}
\alpha & 0 \\
0 & \alpha
\end{array}\right), \quad|\alpha|=q^{-k}
$$

La fonction $T^{m}, m=0, \ldots$, correspond à la trace de la représentation iréductible $\rho_{m}$ de dimension $m+1$ de $S L(2)$, dont la trace est

$$
\gamma=\left(\begin{array}{cc}
\alpha & 0 \\
0 & \beta
\end{array}\right) \mapsto \operatorname{tr}\left(\rho_{m}(\gamma)\right)=\sum_{0}^{m} \alpha^{k} \beta^{m-k}
$$

L'opérateur $\mathbb{L}(s, \rho)$ de $[\mathrm{FLN}]$ correspond à la fonction invariante sur $S L(2, \mathbb{C})$ donnée par

$$
\gamma \mapsto \frac{1}{\left(1-\alpha^{m} / q^{s}\right)\left(1-\alpha^{m-1} \beta / q^{s}\right) \ldots\left(1-\beta^{m} / q^{s}\right)}=\sum_{n=0}^{\infty} \frac{\operatorname{tr} \rho_{m}^{(n)}(\gamma)}{q^{n s}} .
$$

Il faut donc trouver les coefficients de l'expansion

$$
\rho_{m}^{(n)}=\sum_{k=0}^{\infty} a_{m, k}^{(n)} \rho_{k}
$$


car alors la fonction $K^{\rho_{m},(n)}$ de $[\mathrm{FLN}]$ est

$$
\sum_{k=0}^{\infty} a_{m, k}^{(n)} T^{k} .
$$

Le coefficient $a_{m, k}^{(n)}=0$ pour $k>m n$. Les cas les plus simples sont

$$
\left\{\begin{array}{l}
\sum_{k=0}^{\infty} a_{0, k}^{(n)} T^{k}=T^{0} \\
\sum_{k=0}^{\infty} a_{1, k}^{(n)} T^{k}=T^{n} \\
\sum_{k=0}^{\infty} a_{2, k}^{(n)} T^{k}=T^{2 n}+T_{2}^{2 n-4}+T_{4}^{2 n-8}+\cdots+ \begin{cases}T_{n-1}^{2}, & n \equiv 1(\bmod 2), \\
T_{n-2}^{0}, & n \equiv 0(\bmod 2) .\end{cases}
\end{array}\right.
$$

Pour calculer les coefficients $a_{m, k}^{(n)}$ systématiquement et à tour de rôle, utilisons la base habituelle $\left\{\mathfrak{x}_{0}, \ldots, \mathfrak{x}_{m}\right\}$ de $\rho_{m}$ à poids $\lambda_{m-i, i}$

$$
\left(\begin{array}{cc}
\alpha & 0 \\
0 & \beta
\end{array}\right): \mathfrak{x}_{i} \mapsto \alpha^{m-i} \beta^{i} \mathfrak{x}_{i}
$$

donc l'image de la matrice diagonale à valeurs propres $\alpha, \beta$ multiplie le vecteur $\mathfrak{x}_{i}$ par $\alpha^{m-i} \beta^{i}$. Une base de l'espace de $\rho_{m}^{(n)}$ est donnée par les vecteurs

$$
\overbrace{\mathfrak{x}_{0} \otimes \cdots \otimes \mathfrak{x}_{0}}^{l_{0} \text { times }} \otimes \overbrace{\mathfrak{x}_{1} \otimes \cdots \otimes \mathfrak{x}_{1}}^{l_{1} \text { times }} \otimes \cdots \otimes \overbrace{\mathfrak{x}_{m} \otimes \cdots \otimes \mathfrak{x}_{m}}^{l_{m} \text { times }}, \quad l_{0}+l_{1}+\cdots+l_{m}=n,
$$

au nombre de $(n+1) \ldots(n+m) / m$ !, qui est la dimension de $\rho_{m}^{(n)}$. Pour nous rassurer, observons que pour $n=2 n_{1}$ pair, la somme des dimensions à droite dans la dernière ligne de (6.4) est

$$
\left(4 n_{1}+1\right)+\left(4\left(n_{1}-1\right)+1\right)+\cdots+(4+1)+1=4 \frac{n_{1}\left(n_{1}+1\right)}{2}+n_{1}+1=\left(n_{1}+1\right)\left(2 n_{1}+1\right)
$$

qui n'est rien que $((n+1)(n+2)) / 2$. Un calcul semblable est valable pour $n=2 n_{1}+1$.

Puisque l'ensemble de toutes les représentations $\left\{\rho_{0}, \rho_{1} \ldots\right\}$ est l'ensemble des représentations irréductibles de $S L(n)$, nous pouvons en principe déterminer les sous-représentations de $\rho_{m}^{(n)}$ en examinant les vecteurs propres du tore diagonal. Plus précisément, les multiplicités de ses composantes irréductibles, et non pas les sous-espaces sur lesquels elles agissent. Le poids $\lambda_{m n}$, qui est maximal parmi ceux qui apparaissent, est donné par $l_{0}=m, l_{1}=\cdots=l_{m}=0$. Les prochains poids sont donnés par

$$
\left\{\begin{array}{l}
\lambda_{m n-1,1}: l_{0}=n-1, l_{1}=1 ; \\
\lambda_{m n-2,2}: l_{0}=n-2, l_{1}=2 ; l_{0}=n-1, l_{2}=1 ; \\
\lambda_{m n-3,3}: l_{0}=n-3, l_{1}=3 ; l_{0}=n-2, l_{1}=1, l_{2}=1 ; l_{0}=n-1, l_{3}=1 .
\end{array}\right.
$$


Pour $\lambda_{m n-4,4}$ on a, pour $n \geq 4$ et $m \geq 4$, cinq possibilités $l_{0}=n-4, l_{1}=4, l_{0}=n-3, l_{1}=$ $2, l_{2}=1, l_{0}=n-2, l_{2}=2, l_{0}=n-2, l_{1}=1, l_{3}=1, l_{0}=n-1, l_{4}=1$. Les $l_{i}$ qui ne sont pas explicitement donnés sont 0 . Il faut toutefois se rappeler que pour un $n$ donné les possibilités, telles que $n=2, l_{0}=n-3$ pour lesquelles un $l_{i}$ est négatif sont exclues.

En général la suite des premiers éléments $l_{0}=n-k, l_{1}=k$, dans les lignes de (6.5), donne un ensemble qui correspond aux poids de $\rho_{m n}$. La suite $l_{0}=n-k, l_{1}=k-1$, $l_{2}=1$ donne $\rho_{m n-4} \otimes \operatorname{det}^{2}$, où det est la représentation de dimension 1 donnée par le déterminant. Le poids maximal de $\rho_{m n-4} \otimes \operatorname{det}^{2}$ est $\alpha^{2(n-k)}(\alpha \beta)^{k-1} \beta^{2}=(\alpha \beta)^{2} \alpha^{2(n-2)}$. Le premier facteur correspond à une puissance du déterminant, qui n'est d'aucune importance ni pour $S L(2)$ ni pour $P G L(2)$. Pour ce dernier groupe, seulement les représentations de dimension impaire interviennent.

Il y a d'autres suites, celles pour lesquelles $l_{2}=2,3,4, \ldots$ et qui commencent avec $l_{0}=n-l_{2}, l_{1}=0$ et dans lesquelles $l_{0}$ décroît régulièrement, $l_{0} \rightarrow l_{0}-1, l_{1}$ croît, $l_{1} \mapsto l_{1}+1$ et $l_{2}$ est fixé. Lorsque $m>2$ il y a même d'autre suites. Évidemment ces suites s'arrêteront car $l_{0}$ ne peut pas devenir négatif. Donc notre description reste floue et tant que nous n'aurons pas réussi à mener notre étude de la formule de Poisson au point où nous avons une meilleure prise analytique sur la somme de Poisson

$$
\sum_{b \in F}\left\{\hat{\theta}_{f}(b)-\hat{\varphi}_{f}(b)\right\}
$$

il n'est guère utile d'essayer de trouver une expression précise pour les coefficients $a_{m, k}^{(n)}$.

Moi-même, bien que je mise ici aussi bien que dans ma contribution à [FLN] sur la formule de Poisson, je ne sais pas à quoi m'attendre exactement. En principe, nous aurons à examiner pour $s \searrow 1$ la somme d'une série de Dirichlet

$$
\sum_{n} \frac{a_{n}}{n^{s}}
$$

où $n$ parcourt soit les entiers positifs, soit les diviseurs positifs d'une courbe sur un corps fini. À part une fonction holomorphe en $s=1$, la somme est censée donner une somme finie

$$
\sum_{i=1}^{n} b_{i} \zeta_{F, S}(s) L_{i, S}(s)
$$

où les $a_{i}$ sont des constantes, $\zeta_{F}$ la fonction $\zeta$ de $F$ et $\zeta_{F, S}$ sa forme partielle, où le produit n'est pris que sur les places en dehors de $S . L_{i, S}$ est le produit partiel d'une fonction $L$ automorphe sans pôle ni zéro en $s=1$. En particulier il s'agira dans (6.6) d'une fonction avec un pôle d'un ordre imprévisible à $s=1$ et même une partie principale inconnue à tous les égards. Le comportement des coefficients $a_{n}$ lorsque $n \rightarrow \infty$ sera déterminé en large part par celui des coefficients de (6.3) et à son tour influencera le comportement (6.7) lorsque $s \searrow 1$. Il est donc clair que ce n'est pas la détermination précise des coefficients (6.3) dont on aura besoin, mais leur comportement asymptotique. Je suis pourtant incertain 
de savoir quoi faire. Il est toujours possible qu'il serait mieux de revenir aux dérivées logarithmiques.

\section{Un avertissement}

\section{Quelques observations formelles en guise d'avertissement mais aussi d'éclair-} cissement. L'astuce de Getz, que nous avons décrite dans la section $\S 4$ de [FLN] et employée dans le présent article est un peu troublante car le choix de l'ensemble $S^{\prime} \supset S$ est, si non libre, au moins en grande partie arbitraire. En soi il n'y a ici rien de troublant, car les intégrales orbitales, au moins pour $\gamma \in G(F)$ réguliers, se modifient par un facteur constant. Ce n'est qu'en combinaison avec la formule de Poisson qu'elle devient troublante.

Une fois $S^{\prime}$ choisi, l'on peut toujours l'agrandir en remplaçant $S^{\prime}$ par $S^{\prime \prime} \supset S^{\prime}$. Il faut même passer à la limite $S^{\prime \prime} \rightarrow \infty$, dans le sens que $S^{\prime \prime}$ croît de façon à englober toutes les places. Nous avons déjà fait ainsi dans [FLN]. Pour atténuer les inquiétudes, ou plutôt pour attirer l'attention du lecteur sur les questions qu'il reste à résoudre, il est utile d'examiner l'effet d'un agrandissement de $S^{\prime}$, donc d'un replacement de $S^{\prime}$ par $S^{\prime \prime}$. Nous utiliserons les notations de [FLN].

Il y a un analogue pour $\varphi$ de la proposition 5.6 de [FLN]. Pour l'énoncer posons un indice et écrivons pour un $f=\prod f_{v}$ donné,

$$
\varphi_{S^{\prime}}=\prod_{v \in S^{\prime}} \varphi_{v} .
$$

Sa tranformée de Fourier est aussi une fonction sur $\mathbb{A}_{S^{\prime}}$. Dans le lemme suivant il est implicite que la caractéristique globale n'est pas 2. Autrement, les fonctions $\varphi_{v}$ ne sont pas encore définies, même presque partout.

Lemme 7.1. Nous avons

$$
\lim _{S^{\prime \prime} \rightarrow \infty} \hat{\varphi}_{S^{\prime \prime}}(0)=0
$$

Si $S^{\prime \prime}=S^{\prime} \cup\{v\}$ et si $f_{v}$ est la fonction caractéristique de $G\left(\mathcal{O}_{v}\right)$, alors la fonction $\varphi_{S^{\prime \prime}}$ est le produit de la fonction $\varphi_{S^{\prime}}$ et de $\varphi_{v}$ et

$$
\hat{\varphi}_{S^{\prime \prime}}(0)=\hat{\varphi}_{S^{\prime}}(0) \hat{\varphi}_{v}(0)
$$

Selon le calcul de la fonction $f_{v}^{H}$, lorsque $f_{v}^{G}$ est l'élément neutre de l'algèbre de Hecke, $\varphi_{v}(b)$ est donné par l'une des trois possibilités suivantes.

(i) Soit $H$ le tore déployé. Supposons que l'élément $b \in \mathfrak{B}$ est déployé et l'image de $\gamma \in H$ avec des valeurs propres $\lambda^{ \pm 1}$. La valeur $f^{H}(\gamma)=1$ si et seulement si $|\lambda|=1$, donc si et seulement si $b$ est entier et $b^{2}-4$ un carré dans $F^{\times}$. Sinon, $f^{H}(\gamma)=0$. Si $f^{H}(\gamma)=1$, alors, selon le lemme 3.1, $\varphi(b)=\left(1-1 / q_{v}\right)^{-1}\left|b^{2}-4\right|_{v}^{1 / 2}$.

(ii) Si $b$ est non ramifié, donc si $\left|b^{2}-4\right|_{v}=q_{v}^{-2 m}$ avec un exposant non négatif et pair, alors $\varphi(b)=\left(1+1 / q_{v}\right)^{-1}\left|b^{2}-4\right|_{v}^{1 / 2}$.

(iii) Si $b$ est ramifié, alors $\varphi_{v}(b)=0$. 
Calculons

$$
\int_{F_{v}} \varphi_{v}(b) d b
$$

Nous pouvons supposer que la mesure de $\mathcal{O}_{v}$ est 1 . Puisque $b^{2}-4=(b-2)(b+2)$, il y a, modulo l'idéal maximal, $\left(q_{v}+1\right) / 2$ choix de $b$ tels que $\left|b^{2}-4\right|=1$ et $b^{2}-4$ est un carré, parmi lesquels $b \equiv 0$ est une possibilité, et $\left(q_{v}-5\right) / 2$ choix de $b$ tels que $\left|b^{2}-4\right|=1$ et $b^{2}-4$ n'est pas un carré. La contribution de ces points à l'intégrale $\int_{F_{v}} \varphi(b) d b$ est

$$
\frac{1}{2}\left\{\frac{1+1 / q_{v}}{1-1 / q_{v}}+\frac{1-5 / q_{v}}{1+1 / q_{v}}\right\}=\frac{1-2 / q_{v}+3 / q_{v}^{2}}{1-1 / q_{v}^{2}}
$$

Pour les autres points, il faut que $b= \pm(2+x),|x|=q_{v}^{-2 m}<1$, de sorte que $b-4=4 x+x^{2}$. La moitié des $x$ donne (i) et l'autre moitié donne (ii). Pour un $m$ donné, la contribution totale est

$$
\frac{1}{q_{v}^{3 m}}\left(1+\frac{1}{q_{v}}\right)^{-1}
$$

Il s'ensuit que $(7.1)$ est $1-2 / q_{v}+O\left(1 / q_{v}^{2}\right)$. Le lemme en résulte.

La notation a un défaut ou une ambiguité. Au début $S^{\prime}$ fut donné selon le choix de $f$ et l'ensemble agrandi était soit un nouveau $S^{\prime}$ soit un agrandissement $S^{\prime \prime}$ de $S^{\prime}$, qui lui-même ne change pas. Je préfère dans les remarques qui suivent fixer $S^{\prime}$.

Il était déjà évident dans l'article [FLN] qu'il faut à nos fins passer à la limite $S^{\prime \prime} \rightarrow \infty$ car c'est seulement pour la limite que la contribution de $\hat{\theta}(0)$ devient la trace $\pi_{0}(f)$ de la représentation triviale de $S L\left(2, \mathbb{A}_{F}\right)$. Bien que l'on peut passer à cette limite lorsqu'on ne considère qu'un seule terme $\hat{\theta}(0)$ ou $\hat{\varphi}(0)$, il n'en est pas ainsi pour les sommes de Poisson

$$
\sum_{b \in F} \hat{\theta}(b), \quad \sum_{b \in F} \hat{\varphi}(b)
$$

On ne peut pas traiter les limites de ces deux sommes séparément, car elles n'existent pas. Pour le tore déployé, les facteurs $\prod_{v \in S^{\prime}} L(1, \sigma / T)$ vont vers l'infini. Franchement, en ce moment je ne sais pas exactement quoi faire. J'ajouterai quelques observations mais la question de l'existence de la limite

$$
\lim _{S^{\prime \prime} \rightarrow \infty} \sum_{b \neq 0}\left\{\hat{\theta}_{f}(b)-\hat{\varphi}_{f}(b)\right\}
$$

et de sa forme restera ouverte. Cette question est la première, et à mon avis la plus facile, de trois difficultés, deux analytiques, la troisième algébrique, qui empêchent pour le moment l'application efficace des idées de cet article et celui qui l'a précédé. Tout compte fait, je donnerai des suggestions assez précises.

On a $\mathbb{A}_{F}^{S^{\prime}} \subset \mathbb{A}_{F}^{S^{\prime \prime}}$ et, grâce à la condition (4.3), $\mathbb{A}_{F}^{S^{\prime \prime}}=\mathbb{A}_{F}^{S^{\prime}}+F_{S^{\prime \prime}}$. Nous pouvons supposer que l'ensemble $S^{\prime}$ de départ est tel que pour $v \notin S^{\prime}, \mathcal{O}_{v}$ est auto-dual par 
rapport au caractère $\chi_{v}, \chi$ étant le caractère global à la base de toutes les normalisations (cf. [FLN]), et que $f_{v}$ est l'élément neutre dans l'algèbre de Hecke. Réfléchissons sur un passage de $S^{\prime}$ à $S^{\prime \prime}=S^{\prime} \cup\{v\}, v \notin S^{\prime}$. Si $\theta_{v}$ et $\varphi_{v}$ n'étaient que la fonction caractéristique de l'ensemble $\mathcal{O}_{v}$ ou le produit de cette fonction avec une constante, alors $\hat{\theta}_{v}$ et $\hat{\varphi}$ seraient aussi cette fonction de sorte que $\theta(b)=\varphi(b)=0$ pour $b$ dans $F_{S^{\prime \prime}}$, mais non pas dans $F_{S^{\prime}}$, et les sommes de Poisson pour $S^{\prime \prime}$ seraient égales à celles pour $S^{\prime}$.

C'est là exactement la source d'une des difficultés principales. Même si $f_{v}$ est l'élément neutre de l'algèbre de Hecke, les fonctions $\theta_{v}$ et $\varphi_{v}$ ne sont pas des fonctions simples. Pour une place non archimédienne une formule pour la fonction $\varphi_{v}=\varphi_{f_{v}}$ que donne l'élément neutre se déduit immédiatement du lemme $3.1 \mathrm{et,} \mathrm{pour} \mathrm{la} \mathrm{fonction} \theta_{v}=\theta_{f_{v}}$, des formules (3.6), où l'entier $m$ est alors 0 .

Décrivons ces fonctions $\theta_{v}$ et $\varphi_{v}$ qualitativement mais, pour simplicité, supposons que la caractéristique résiduelle n'est pas 2 . Elles sont les sommes d'une fonction lisse $\theta_{v}^{\mathrm{sm}}$ ou $\varphi_{v}^{\mathrm{sm}}$ à support compact et pour chacune des quatre classes de tores, $T_{v}=T^{\mathrm{sp}}, T_{v}=T^{\mathrm{unr}}$, $T_{v}=T_{1}^{\mathrm{ram}}, T_{v}=T_{2}^{\mathrm{ram}}$, de fonctions $\theta_{ \pm}^{T_{v}}$ ou $\varphi_{ \pm}^{T_{v}}$ supportées sur un ensemble

$$
U_{ \pm}^{T_{v}}=\left\{b|| b \mp 2\left|\leq \epsilon_{ \pm}^{T_{v}},\right| b \pm 2 \mid<1, b \mp 2 \sim T_{v}\right\}
$$

où elles sont égales à $c_{ \pm}^{T_{v}}(\theta)|\Delta(b)|$ ou, selon le cas, $c_{ \pm}^{T_{v}}(\varphi)|\Delta(b)|$ et où $c_{ \pm}^{T_{v}}(\theta)$ et $c_{ \pm}^{T_{v}}(\varphi)$ sont des constantes. La notation $x \sim T_{v}$ signifie que $T_{v}$ est rattaché à l'extension $F(\sqrt{x})$. Pour simplifier la notation nous admettons les $x \in\left(F_{v}^{\times}\right)^{2}$. Nous ne demandons donc que $F(\sqrt{x})$ soit un corps. Nous avons calculé les transformées de Fourier de telles fonctions dans la section $\S 4$.

On aura $c_{ \pm}^{T^{\mathrm{sp}}}(\varphi)=\theta_{v}^{\mathrm{sm}}( \pm 2)$ et $c_{ \pm}^{T^{\mathrm{sp}}}(\theta)=0$. En plus, selon le lemme 2.2.2, les constantes $c_{ \pm}^{T_{v}}(\theta), T_{v}=T^{\mathrm{unr}}, T_{i}^{\mathrm{ram}}, i=1,2$, se déterminent, l'une, l'autre. Il me semble, par contre, que les trois autres constantes sont indépendantes de ces cinq, car on peut prendre pour $f^{G}$ un coefficient matriciel d'une représentation fortement cuspidale de $G\left(F_{v}\right)$. Ce n'est donc pas cette description qualitative qui donnerait les relations pertinentes entre $\theta_{v}$ et $\varphi_{v}$ qui sont, après tout, des fonctions linéaires, l'une de l'autre.

À nos fins, qui sont toujours informelles, nous pouvons supposer qu'aux places archimédiennes $f_{v}^{G}$ a son support dans l'ensemble des éléments semi-simples et réguliers. Alors $\theta_{v}$ et $\varphi_{v}$ sont lisses à support compact disjoint de $\{ \pm 2\}$ ou, mieux, simplement lisses partout.

Aux places en dehors de $S^{\prime}$, la fonction $f_{v}$ est l'élément neutre dans l'algèbre de Hecke et des descriptions plus précises de $\theta_{v}$ et $\varphi_{v}$ sont possibles. Puisque cela ne change que peu l'argument que je propose, un argument qui reste pour le moment très flou, je suppose qu'à tout place $v \in S^{\prime}$ les fonctions $\theta_{v}$ et $\varphi_{v}$ sont à support compact dans le complément de $\pm 2 \mathrm{ou}$, encore, lisses partout. Pour cela il suffit de supposer que le support des fonctions $f_{v}^{G}$ ne contient pas des éléments $\pm g, g$ étant unipotent. Pour les $v$ non archimédiens pour lesquels ceci n'est pas le cas, il faut traiter $\theta_{v}$ et $\varphi_{v}$ comme on les traite dans l'argument suivant, mais en acceptant que les constantes $c_{ \pm}^{T_{v}}$ et les supports de la formule (7.3) sont arbitraires, et que les constantes ne sont pas les mêmes pour les deux tores ramifiés. Cela entraînera des complications mineures. 
L'ensemble $S^{\prime}$ est donné. Nous le remplaçons par $S^{\prime \prime} \supset S^{\prime}$ et voulons montrer que la limite (7.2) existe. La limite est comprise dans le sens que $S^{\prime \prime}$ est donné comme la réunion des places non archimédiennes et l'ensemble des places telle que $q_{v} \leq N, N \rightarrow \infty$. Puisque les termes de (7.2) sont compliqués, je préférerai ne traiter que le corps des nombres rationels, mais pour les quelques calculs locaux qui restent, nous pouvons continuer à travailler en général, sauf pour l'exclusion des places à caractéristique résiduelle 2. Alors $b$ parcourt un ensemble de nombres à dénominateurs qui sont des produits de nombres premiers qui appartiennent à $S^{\prime \prime}$ et dont les valeurs absolues comme nombres réels sont bornées. En fait, il faut admettre au dénominateur des puissances de nombres premiers dans $S^{\prime}$ mais avec des exposants bornés. Il est plus simple de supposer que le support $\operatorname{des} f_{v}, v \in S^{\prime}$, est suffisamment petit pour que ces exposants soient 0 . Il s'agit d'une contrainte légère sans aucune importance.

Pour avoir une notation, pour une classe donnée $\alpha$ de $F^{\times}$suivant $\left(F^{\times}\right)^{2}$, soit $\xi_{\alpha}^{ \pm}$le produit de la fonction caractéristique de $\pm 2+\alpha \cap \mathcal{O}_{F}$ et de $|\Delta|$. Soit $\eta$ la fonction caractéristique de $\mathcal{O}_{v}$. En classifiant les $\alpha$ selon les tores auxquels ils sont rattachés, nous introduisons les notations $\alpha^{\mathrm{sp}}, \alpha^{\mathrm{unr}}, \alpha_{1}^{\mathrm{ram}}, \alpha_{2}^{\mathrm{ram}}$ et les notations $\xi^{\mathrm{sp}}, \xi^{\mathrm{unr}}, \xi_{1}^{\mathrm{ram}}, \xi_{2}^{\mathrm{ram}}$. En dehors de $S^{\prime}$ la fonction $\theta_{v}$ est donnée par la somme

$$
\left(1+\frac{1}{q_{v}}\right) \eta-\frac{2}{q_{v}} \xi^{\mathrm{unr}}-q_{v}^{-1 / 2}\left(1+\frac{1}{q_{v}}\right)\left(\xi_{1}^{\mathrm{ram}}+\xi_{2}^{\mathrm{ram}}\right) .
$$

Pour les fonctions $\varphi_{v}$ il y a une formule semblable,

$$
\varphi_{v}=\left(1+\frac{1}{q_{v}}\right) \xi^{\mathrm{sp}}+\left(1-\frac{1}{q_{v}}\right) \xi^{\mathrm{unr}} .
$$

Ces formules et celles que nous avons données en fin de la démonstration du lemme 4.3 nous permettront de calculer $\hat{\theta}_{v}$ et $\hat{\varphi}_{v}$. L'exposant $\lambda$ de ce lemme est maintenant $3 / 2$. La fonction $\hat{\eta}$ est égale à $\eta$. La fonction $\hat{\xi}^{\text {sp }}$ est donnée par les formules (4.17.a) et (4.17.b). Si $a \in \mathcal{O}_{v},|a|_{v}=1$ et $a$ n'est pas un carré, alors $\hat{\xi}^{\mathrm{unr}}(x)=\hat{\xi}^{\mathrm{sp}}(a x)$ de sorte que ces deux formules donnent en plus une formule pour $\hat{\xi}^{\mathrm{unr}}$. De la même façon, si $\left|b_{i}\right|=q^{-1}$, $\alpha_{i}^{\mathrm{ram}} / b_{i} \in\left(F^{\times}\right)^{2}$, alors $\xi_{i}^{\mathrm{ram}}(x)=\xi^{\mathrm{sp}}\left(x / b_{i}\right), i=1,2$.

En principe donc, nous avons, en dehors de $S^{\prime}$, tous les renseignements nécessaires ou possibles, même s'ils sont en partie inutiles, sur les functions qui interviennent dans (7.4.a) et (7.4.b). Je n'ai en ce moment ni la force ni le courage de poursuivre l'étude de la somme de Poisson pour $\theta_{S^{\prime \prime}}$ ou pour $\varphi_{S^{\prime \prime}}$ mais avant de fermer boutique pour un temps de repos je veux expliquer brièvement mes espoirs, toujours incertains. Pour le corps des nombres rationels, la somme sur $b \neq 0$ de $\hat{\theta}(b)$ ou de $\hat{\varphi}$, est essentiellement une somme sur des nombres rationnels

$$
b=\frac{a}{\prod_{v \in S^{\prime \prime}-S^{\prime}} p_{v}^{m_{v}}}, \quad m_{v} \geq 0
$$

pour lesquels le dénominateur est un produit sur l'ensemble des places de $S^{\prime \prime}$ qui n'appartiennent pas à $S^{\prime}$ et le numérateur a est premier au dénominateur. Ces nombres sont de 
plus majorés par une constante aux places de $S^{\prime}$, car, en dehors de cet ensemble de $b$, $\hat{\theta}=\hat{\theta}_{S^{\prime \prime}}$ et $\hat{\varphi}_{v}=\hat{\varphi}_{v}$ sont les fonctions caractéristiques de $\mathcal{O}_{v}$. Nous excluons $b=0$. Il est préférable de prendre comme indices $M$ les couples formés d'un ensemble $R \subset\left(S^{\prime \prime}-S^{\prime}\right)$ et un ensemble $\left\{m_{v} \mid v \in R\right\}$ et de rattacher $b=a / \prod_{v \in S^{\prime \prime}-S^{\prime}} p_{v}^{m_{v}}$ à l'indice $M$ donné par $\left\{v \in S^{\prime \prime}-S^{\prime} \mid m_{v}>0\right\}$. Chaque $b$ est rattaché à un $M$ unique. Nous écrivons $b \in M$.

Une difficulté importante, c'est que même si on réussit à majorer des différences $\hat{\theta}_{v}-\hat{\varphi}_{v}$ à toutes les places, cela ne donne pas assez de renseignements pour les produits infinis de (7.2). Ce que j'envisage est une majoration de la forme

$$
\left.\sum_{b \in M}\left(\hat{\theta}_{S^{\prime \prime}}(b)-\hat{\varphi}_{S^{\prime \prime}} b\right)\right)=O\left(\prod_{v \in R} \frac{1}{p_{v}^{\lambda m_{v}}}\right), \quad \lambda>1
$$

Quels sont les liens entre les fonctions $\theta_{f}$ et $\varphi_{f}$, deux fonctions qui se déterminent, l'une, l'autre, qui nous permettent de majorer la différence (7.2)? Une possibilité séduisante, au moins pour une place à caractéristique résiduelle différente de 2, est l'égalité,

$$
\varphi_{v}(0)=\theta_{v}(0)
$$

Elle est même valable souvent. Si l'image dans la base de Steinberg-Hitchin de $\gamma \in G(F)$ est 0 , alors ses valeurs propres sont $\pm \sqrt{-1}$ de sorte que $|\Delta(\gamma)|=1$ lorsque la caractéristique résiduelle est impaire. Donc, si elles appartiennent à $F_{v}$, l'égalité $(7.7)$ est certainement valable, car $\varphi_{v}(b)=|\Delta(b)|_{v} \theta_{v}(b)$ lorsque $b$ est l'image d'un élément déployé. En plus, pour un corps non archimédien et une fonction sphérique, donc une des fonctions $T_{m}$, elle découlé des quatre formules (3.6), (3.9), (3.9.a) et (3.9.b). Il semble que l'égalité, qui revient en général à l'égalité

$$
\operatorname{Orb}(\gamma, f)=f^{H}(\gamma)
$$

pour le $\gamma$ en question, est toutefois en général fausse. Il y a cependant une lueur d'espoir que lorsque l'on aura enfin maîtrisé les constructions de cet article une autre forme de (7.7) mieux adaptée à nos fins, et, en plus, vraie, aura été trouvée. Il faudra aussi une forme pour la caractéristique résiduelle 2 .

L'égalité (7.7) affirme que

$$
\int_{\mathfrak{A}_{v}} \hat{\theta}_{v}(b)=\int_{\mathfrak{A}_{v}} \hat{\varphi}_{v}(b) .
$$

Dans (7.6) la somme sur $b$ de la différence $\hat{\theta}_{S^{\prime \prime}}-\hat{\varphi}_{S^{\prime \prime}}$ est, pour chaque $\prod p_{v}^{m_{v}}$ une somme sur $a$. La valeur de cette somme sur $a$ est approximée par le produit de $\alpha=\prod_{v \in S^{\prime}} p_{v}^{m_{v}}$ et la différence entre les intégrales de $\hat{\theta}$ et $\varphi$ sur $\prod_{v \in S^{\prime}} F_{v}$. La différence entre les sommes et la différence des intégrales doit être de l'ordre de $\alpha^{-1}=1 / \prod_{v \in S^{\prime}} p_{v}$ Donc à un terme d'ordre 1 près, la somme sur $a$ peut être remplacée par la différence des intégrales, une différence qui serait, avec l'égalité (7.7), égale à 0 . Il y a en plus une majoration $\prod_{v \in R} 1 / p_{v}^{3 / 2}$ qui 
vient des majorations pour $\hat{\theta}_{v}$ et $\hat{\varphi}_{v}$ de la section $\S 4$. Si je ne me trompe pas, aux places de $S_{R}^{\prime \prime}$ ces deux fonctions seront toutes les deux approximativement 1. De toutes façons, cela donnerait une majoration

$$
\sum_{R} \prod_{v \in R} 1 / p_{v}^{3 / 2}
$$

qui resterait bornée lorsque $S^{\prime \prime} \rightarrow \infty$.

Je ne tiens pas beaucoup à la forme précise de ces suggestions. Elles sont inclues pour encourager les lecteurs et les lectrices de chercher eux-mêmes et elles-mêmes une solution des problèmes qui restent. J'accepte qu'elles puissent sembler farfelues mais elles sont les seules que j'ai trouvées. Depuis le début de mes réflexions sur cet article et même de mes conversations avec Ngô Bao Chaû et Edward Frenkel, j'éprouvais un malaise car je craignais que l'on suivait une piste douteuse, mais chaque difficulté fut surmontée, et chaque fois que je me suis arrêté pour jeter un coup d'oeil sur le sentier traversé j'ai eu le sentiment qu'il était, malgré tout, assez bien visible parmi les broussailles. J'espère qu'il sera ainsi avec les difficultés que je viens de décrire.

\section{L'avenir}

8. L'avenir. L'avenir présentera d'autres difficultés importantes. Essayons de les prévoir. Les difficultés sont de deux sortes, d'abord, même après avoir résolu des difficultés du passage à la limite $S^{\prime \prime} \rightarrow \infty$, il restera à examiner le comportement asymptotique de la somme

$$
\sum_{\pi^{\mathrm{st}} \in \operatorname{Ram}} L(s, \pi, \rho), \quad \pi \in \pi^{\mathrm{st}},
$$

ou, car rien n'est encore certain,

$$
-\sum_{\pi^{\mathrm{st}} \in \operatorname{Ram}} \frac{L^{\prime}(s, \pi, \rho)}{L(s, \pi, \rho)}, \quad \pi \in \pi^{\mathrm{st}}
$$

lorsque $s \rightarrow 1$. L'ensemble Ram est l'ensemble des représentations de $G$ de type Ramanujan, donc celles qui dans le cadre introduit par Arthur correspondent à un paramètre pour lequel l'homomorphisme $S L(2) \rightarrow \hat{G}$ est trivial. Alors, comme expliqué dans [FLN], le pôle de $L(s, \pi, \rho)$ dépendra de $\rho_{H}=\rho \circ \psi$ et son ordre $n_{H}$ sera la multiplicité de la représentation triviale dans $\rho_{H}$. Pour pouvoir parler de cette possibilité dans l'avenir sans répéter sa description, j'ai proposé ailleurs informellement d'appeler $\pi^{\text {st }}$ hadronique (épais) si ${ }^{\lambda} H={ }^{L} G$. Il arrivera sans doute parfois, quoique exceptionnellement, que $\pi_{G}^{\text {st }}$ soit l'image de $\pi_{H}^{\text {st }}$ sous $\phi_{G, H}:{ }^{L} H \rightarrow{ }^{L} G$ et sous $\phi_{H}^{\prime}:{ }^{L} H \rightarrow{ }^{L} G$, mais que $\phi_{H}$ et $\phi_{H}^{\prime}$ ne sont pas conjugués ou encore que $\pi_{G}^{\text {st }}$ soit l'image d'un $\pi_{H}$ qui n'est pas hadronique pour $H$. Mais nous pouvons mettre ce genre de difficultés à côté pour le moment car, bien que les conséquences seront fascinantes à cause de leurs liens avec les multiplicités $m_{G}^{\text {st }}$, elles se résoudront probablement d'elles-mêmes. 
Si $\pi_{G}^{\text {st }}$, supposé de type Ramanujan, est l'image fonctorielle de $\pi_{H}^{\text {st }}$ - supposé, pour rendre le commentaire plus transparent, hadronique - par rapport à $\phi_{G, H}$ et la représentation $\rho_{H}^{\text {comp }}$ est complémentaire à la partie triviale de $\rho_{H}$, on aura

$$
L\left(s, \pi_{G}, \rho_{G}\right)=\zeta_{F}(s)^{m_{0}} L\left(s, \pi_{H}, \rho_{H}^{\mathrm{comp}}\right), \quad \rho_{H}=\rho_{G} \circ \phi_{G, H}, \quad m_{0}=\operatorname{dim} \rho_{G}-\operatorname{dim} \rho_{H}^{\operatorname{comp}} .
$$

On s'attend de, ou plutôt on espère, pouvoir déterminer le comportement asymptotique - par exemple, l'ordre du pôle — de

$$
\sum_{\pi_{H}^{\mathrm{st}} \in \operatorname{Ram}_{H}} L\left(s, \pi_{H}, \rho_{H}^{\mathrm{comp}}\right)
$$

lorsque $s \rightarrow 1$ à partir de la formule des traces stable pour $H$. Il y aura certainement des ambiguités à résoudre pour les représentations $\pi_{H}^{\text {st }}$ qui ne sont pas hadroniques. Avec ou sans les représentations hadroniques, on aura besoin d'une expression plus concrète pour $(8.2)$.

Si, par exemple, $H=\{1\}$ alors $L\left(s, \pi_{H}, \rho_{H}^{\text {comp }}\right)$ est une fonction $L$ d'Artin et on cherche une expression pour la valeur en $s=1$ de cette fonction et peut-être aussi de quelques dérivées. Grâce au théorème de Brauer on est ramené aux fonctions $L$ abéliennes dont les valeurs en $s=0$ ont été beaucoup étudiées.

Nous sommes habitués par la théorie du corps de classes au comportement asymptotique de $\zeta_{F}(s)$ découvert, comme un fait général, par Dedekind et Frobenius, à savoir

$$
\zeta_{F}(s)=\sum_{\mathfrak{a}} \frac{1}{\mathrm{Na}^{s}} \sim \sum_{\mathfrak{p}} \frac{1}{\mathrm{~Np}^{s}} \sim \frac{C}{s-1},
$$

lorsque $s \searrow 1$. Ce comportement asymptotique est lié à celui de

$$
-\frac{\zeta_{F}^{\prime}(s)}{\zeta_{F}(s)} \sim \sum_{\mathfrak{p}} \frac{\ln \mathrm{Np}}{\mathrm{Np}^{s}} \sim \frac{1}{s-1}
$$

car

$$
\begin{aligned}
\zeta_{F}(s) & =\exp \left(\int \frac{\zeta_{F}^{\prime}(s)}{\zeta_{F}(s)}\right) \\
& =C \exp \left(-\ln (s-1)+\sum_{i=1}^{\infty} \int \frac{\alpha_{i}}{i+1}(s-1)^{i}\right) \\
& =\frac{C}{s-1} \exp \left(\sum_{i=1}^{\infty} \alpha_{i}(s-1)^{i}\right),
\end{aligned}
$$

si

$$
\frac{\zeta_{F}^{\prime}(s)}{\zeta_{F}(s)}=-\frac{1}{s-1}+\sum_{i=1}^{\infty} \alpha_{i}(i+1)(s-1)^{i}
$$


Il résulte de l'équation (8.5) que pour exprimer le comportement asymptotique des puissances $\zeta_{F}^{m}(s)$ on a besoin de tous les coefficients $\alpha_{i}$ de (8.6). Au premier abord cela me semble désespérant.

Le cas primaire de (8.2) est le cas où $H=G$ et (8.2) n'est alors que (8.1). Dans cet article le groupe $G$ est $S L(2)$. J'ai proposé, pour ce groupe, et implicitement pour tout groupe réductif, de poursuivre l'analyse de (8.2) en utilisant la formule de Poisson jusqu'au point où on a entre les mains une expression concrète et arithmétique pour celui-ci.

Puisque le facteur $\zeta_{F}^{m_{0}}$ est déjà un obstacle apparemment insurmontable on se demande s'il vaut la peine de continuer. Je persiste néanmoins. Pour un sceptique la possibilité offerte par la base de Steinberg-Hitchin d'utiliser la formule de Poisson pour surmonter des difficultés qui ont empêché l'application de la formule des traces à la fonctorialité en général pendant les quatre dernières décennies et qui n'ont permis que des applications dans le cadre de l'endoscopie, tordue ou non, peut sembler un miroir à alouettes. Pour ma part, je le trouve difficile de croire que toute l'évidence disponible n'est qu'un leurre.

Il n'en reste pas moins que même après avoir trouvé une façon pour passer à la limite $S^{\prime \prime} \rightarrow \infty$ pour un $f$ donné, de sorte que nous ayons sous la main la formule des traces transformée par la formule de Poisson, il faudra prendre pour $f$ les coefficients $f_{n}$ de (5.13) ou même $f(s)$ lui-même et examiner le comportement de sa trace stable lorsque $s \searrow 1$. Il s'agit de la deuxième des difficultés analytiques majeures. Pour la surmonter, pour acquérir l'expérience nécessaire, il faut à mon avis l'aborder doucement en commençant avec les cas les plus simple, par exemple, pour $G=S L(2)$ et $\operatorname{dim} \rho=3$ ou, en changeant légèrement le cadre, pour $G=P G L(2)$ et $\operatorname{dim} \rho=2$.

Pour la théorie classique du corps de classe, le groupe $G$ est égal à $G L(1)$. Selon l'article [FLN], il y a une différence majeure entre les bases de Steinberg-Hitchin pour les deux groupes, $G L(1)$ et $S L(2)$. Pour le deuxième la base est le groupe additif de la ligne droite pour lequel la transformée de Fourier exprime une dualité fondamentale de la théorie des nombres analytique; pour le premier groupe, sa base est le groupe multiplicatif, donc $G L(1, F)$ lui-même, pour lequel la transformée de Fourier ne réussit qu'à défaire la formule des traces et à nous retourner au spectre de $G L(1, F) \backslash G L\left(1, \mathbb{A}_{F}\right)$. Par conséquent, elle est inutile à nos fins. Par contre, examiner, pour le groupe $G L(1)$ la trace des opérateurs de Hecke $f_{n}$ ou celle de $f(s)$ en utilisant directement la formule des traces abélienne est assez facile. Il s'agit, par exemple, de trouver le nombre d'idéaux premiers dans une classe à gauche par rapport à un sous-groupe d'indice fini de $F^{\times} \backslash \mathbb{A}_{F}^{\times}$. Pour $S L(2)$ les calculs, surtout après avoir passé aux transformées de Fourier sur la base de Steinberg-Hitchin, seront bien moins transparents de sorte que le deuxième problème analytique sera peutêtre aussi en partie un problème de géométrie algébrique.

Pour la théorie du corps de classes on compare ce nombre avec le nombre d'extensions finies et abéliennes du corps $F$ à ramification donnée. C'est un calcul qui est la clé de la démonstration des théorèmes fondamentaux de cette théorie, un calcul dans lequel intervient directement la formule des traces abélienne et qui est très difficile $([\mathrm{H}])$. Il me semble qu'il faut essayer de faire une comparison de ce genre pour un groupe général, en commençant avec $S L(2)$, donc de compter le nombre d'extensions à groupe donné et à ramification donnée. Pour la théorie des corps de classes on commence en faisant quelques simplifications pour arriver au cas des extensions cycliques de degré premier $l$ sur un corps 
de base qui contient les racines $l$-ièmes de l'unité. Il est facile de construire ces extensions. Ce sont les extensions $\sqrt[l]{\alpha}, \alpha \neq 0, \alpha \in F$, quoiqu'il faut exclure les $\alpha=\beta^{l}, \beta \in F$. Le groupe $G L(1, F)$ agit sur $F^{\times}$par $\beta: \alpha \mapsto \alpha \beta^{l}$ et ces extensions correspondent aux orbites de $G L(1, F)$ dans $F^{\times}$, sauf que l'orbite du point 1 est exclue. Le comptage, qui n'est pas facile, doit aussi tenir compte de la ramification donnée. Il faut comprendre en particulier, comme dans $[\mathrm{H}]$, pour un $\alpha$ donné quelle est la ramification de $F(\sqrt[l]{\alpha})$.

Il est facile de proposer des constructions semblables pour d'autres groupes finis $\mathfrak{G}$ et des extensions $K$ de $F$ et un isomorphisme $\operatorname{Gal}(K / F) \simeq \mathfrak{G}, \sigma \mapsto g$. Supposons, par exemple, que $\mathfrak{G}$ est simple et que la représentation irréductible $r$ de $G$ est définie sur $F$. Soient $d$ le degré de $r$ et $n(\mathfrak{G})$ le nombre d'éléments de $\mathfrak{G}$. Si $K / F$ est une extension galoisienne de groupe de Galois $\mathfrak{G}$ — et le théorème de Hilbert affirme que de telles extensions existent alors grâce à l'existence d'une base normale de $K / F$ on peut trouver des éléments $x_{i, j} \in K$ tels que $X=\left(x_{i, j}\right)$ est une matrice inversible et

$$
{ }^{\sigma} X=r(\sigma) X, \quad \sigma \in \mathfrak{G}
$$

L'action de $\mathfrak{G}$ est à gauche. Une base normale $\left\{{ }^{\sigma} x \mid \sigma \in \operatorname{Gal}(K / F)\right\}, x \in K$, n'est rien d'autre qu'une façon de réaliser explicitement un isomorphisme entre la représentation $\sigma: y \in K \mapsto{ }^{\sigma} y$ du groupe de Galois $\mathfrak{G}$ sur l'espace vectoriel $K$ sur $F$ et la représentation régulière. Les colonnes $\left(x_{1, j}, x_{2, j}, \ldots, x_{d, j}\right)^{t}$ ne sont alors que des bases de $d$ réalisations indépendantes de la représentation $r$ dans la représentation de $\mathfrak{G}$ sur $K$. Il y a une action transitive du groupe $G L(d, F)$ sur l'ensemble $\mathfrak{X}$ de $X$ qui satisfont aux équations (8.7), $X \mapsto X A$, avec $A \in G L(d, F)$.

Nous pouvons identifier les matrices $X$ aux coefficients dans $\bar{F}^{\text {sep }}$ avec des fonctions bilinéaires sur le produit $V \otimes V$, où $V=\left\{a=\left(a_{1}, \ldots, a_{d}\right)\right\}$ est un espace vectoriel $V$ de dimension $d$ sur $F$.

$$
X: a \otimes b \mapsto \sum_{i, j} a_{i} x_{i, j} b_{j}=a X b^{t}
$$

où $b^{t}$ est la transposée de $b$. Évidemment,

$$
\sigma\left(a X b^{t}\right)=\operatorname{ar}(\sigma) X b^{t}
$$

Pour n'importe quelle matrice $X$ de taille $d$, les $n=n(G)$ fonctions symétriques élémentaires des nombres $\left\{a r(\sigma) X b^{t} \mid \sigma \in \mathfrak{G}\right\}$ sont des polynômes $P_{i}(a, b), i=1, \ldots, n$ en les coefficients $a_{1}, \ldots, a_{d}$ et $b_{1}, \ldots, b_{d}$ de $a$ et $b$. L'ensemble des coefficients de tous les $P_{i}(a, b)$, obtenus en exprimant chaque $P_{i}(a, b)$ comme une combinaison linéaire de monômes en $a_{1}, \ldots, a_{d}$ et $b_{1}, \ldots, b_{d}$, est un ensemble fini $\left\{F_{1}, \ldots, F_{M}\right\}$ de polynômes en les coefficients de $X$ qui définit une application algébrique

$$
\mathcal{F}: X \rightarrow\left(F_{1}(X), \ldots, F_{M}(X)\right)
$$

de l'espace des matrices carrés de rang $d$ dans un espace vectoriel de dimension $M$. L'image dans le sens de la géométrie algébrique est une variété algébrique $\mathfrak{F}$. Il est évident que 
l'action $X \rightarrow X A$ définit une action linéaire sur cet espace vectoriel compatible avec $\mathcal{F}$. Soit

$$
\mathcal{P}_{(a, b)}(T)=T^{n}-P_{1}(a, b) T^{n-1}+-\cdots+(-1)^{d} P_{n}(a, b) .
$$

C'est un polynôme en $T$ dont les coefficients sont des polynômes en les coefficients de $(a, b)$. Si $X$ à coefficients dans $K$ a été choisi pour satisfaire à (8.7), le corps $K$ est engendré par les racines de ces polynômes lorsque les coefficients de $a, b$ parcourent $F$. En particulier $X$ appartient à l'ensemble des matrices à coefficients dans $\bar{F}^{\text {sep }}$ pour lesquelles il existe des $(a, b)$ tels que $\mathcal{P}_{(a, b)}$ n'a pas de racine multiple, donc pour lesquelles le discriminant de $\mathcal{P}_{(a, b)}$ n'est pas égal à zéro comme polynôme en $(a, b)$. Il en résulte que cet ensemble n'est pas vide.

Nous pouvons introduire les polynômes $\mathcal{P}_{(a, b)}$ pour n'importe quelle matrice $X$ à coefficients dans $\bar{F}^{\text {sep }}$. L'application $(a, b) \rightarrow \mathcal{P}$ est une application algébrique et nous pouvons ne considérer que l'ensemble ouvert dans le sens de Zariski de l'espace vectoriel de matrices carrées de rang $d$ à coefficients dans $\bar{F}^{\text {reg }}$ telles que $P_{(a, b)}$ n'a pas de racine multiple pour au moins une paire $(a, b)$. Nous supposons désormais que $X$ à coefficients dans $\bar{F}^{\text {reg }}$ satisfait à cette condition et choisissons $a$ et $b$ à coefficients dans $F$ en sorte que $P_{(a, b)}$ n'a pas de racine multiple. Pour un tel $(a, b), a X b^{t}$ est une racine $\alpha$ de $P_{(a, b)}$ et pour chaque autre racine $\beta$ il y au plus un seul $g=g_{a, b}$ dans $\mathfrak{G}$ tel que $\beta=\operatorname{ar}(g) X b^{t}$. En particulier, si $\sigma \in \operatorname{Gal}\left(\bar{F}^{\text {reg }}\right)$ il y un seul $g=g(\sigma)=g_{a, b}(\sigma)$ tel que $\sigma\left(a X b^{t}\right)=\operatorname{ar}(g) X b^{t}$.

Fixons $\sigma$ et $b$ tels que l'ensemble $R_{b}$ de $a$ pour lesquels $\mathcal{P}_{(a, b)}$ n'a pas de racine multiple n'est pas vide. Pour chaque $g \in \mathfrak{G}$ soit $R_{b}^{g}$, l'ensemble des $a$ tels que $g_{a, b}(\sigma)=g$. Si $a$ et $a^{\prime}$ et $a+a^{\prime}$ appartiennent à $R_{b}^{g}$ alors

$$
\left.\sigma\left(\left(a+a^{\prime}\right) X b^{t}\right)\right)=\sigma\left(a X b^{t}\right)+\sigma\left(a^{\prime} X b^{t}\right)=a r(g) X b^{t}+a^{\prime} r(g) X b^{t}=\left(a+a^{\prime}\right) r(g) X b^{t},
$$

de sorte que $a+a^{\prime} \in R_{b}^{g}$, au moins si $\mathcal{P}_{\left(a+a^{\prime}, b\right)}$ n'a pas de racine multiple. Un argument semblable montre que si $a \in R_{b}^{g}, a^{\prime}=\lambda a, \lambda \in F^{\times}$et $\lambda \neq 0$, alors $a^{\prime} \in R_{b}^{g}$. Soit $\tilde{R}_{b}$ l'ensemble des $a$ pour lesquels $\sigma\left(a X b^{t}\right)=\operatorname{ar}(g) X b^{t}$ mais pour lesquels des racines multiples de $\mathcal{P}_{(a, b)}$ sont admises. Alors $R_{b}^{g} \subset \tilde{R}_{b}^{g}, R_{b}^{g} \cap R_{b}^{g^{\prime}}=\varnothing$ si $g \neq g^{\prime}$, et la réunion des ensembles $\tilde{R}_{b}^{g}, g \in \mathfrak{G}$, est l'ensemble de tous les $a$. En plus, l'ensemble $R_{b}^{g}$ est un sous-espace vectoriel sur $F$, un corps infini, de l'espace $V$. Il en suit immédiatement que l'ensemble $R_{b}^{g}$ n'est vide que pour un seul $g$. Alors $\tilde{R}_{b}^{g}=V$. Nous posons $g=g_{b}$. Le même argument montre que $g$ est indépendant de $b$. Avec un argument supplémentaire mais tout à fait semblable on conclut que $\sigma \mapsto g$ est un homomorphisme du groupe de Galois dans $\mathfrak{G}$. Il n'est pas nécessairement surjectif.

Pour le groupe $\mathfrak{G}=\mathbb{Z}_{l}$ et $r: m \mapsto \zeta^{l}, m \in \mathbb{Z}(\bmod l), \zeta$ une racine primitive de 1 , le degré $d$ est 1 , la base normale est $1, \mu=\sqrt[l]{\alpha}, \mu^{2}, \ldots, \mu^{l-1}, \mu^{l}=\alpha \in F^{\times}$On a $X=\mu$. L'ensemble ${ }^{\sigma} X$ est $\left\{\mu, \zeta \mu, \ldots, \zeta^{l-1} \mu\right\}$ et

$$
\prod_{m=0}^{l-1}\left(T-\zeta^{m} a b \mu\right)=T^{l}-\alpha a^{l} b^{l},
$$


de sorte que $M=1, F_{1}(X)=X^{M}$ et la variété $\mathfrak{F}$ est simplement la variété affine de dimension 1.

Dans ce cas la variété $\mathfrak{F}$ est simple mais l'étude des points sur $F$, ou plutôt de leurs orbites par rapport à l'action de $G L(1)$, surtout du point de vue de leur ramification est très difficile (cf. $[\mathrm{H}]$ ) mais indispensable pour le développement de la théorie des corps de classes. Le faire pour d'autres groupes que $\mathbb{Z}_{l}$ et comparer les résultats avec le comportement asymptotique de (8.2) est pour moi la troisième difficulté majeure qui sera à surmonter.

Observons qu'il y aura à la toute fin, lorsqu'on entame les groupes généraux et essaie de construire une théorie complète, un enchevêtrement du cas Artin, donc du cas où $G=\{1\}$, et du cas où $G$ est un groupe continu, car le groupe ${ }^{L} G$ est de nécessité souvent un mélange de sa composante connexe $\hat{G}$ et d'un groupe de Galois fini $\operatorname{Gal}(K / F)$. Pour le groupe $S L(2)$ on peut éviter les complications qui en découlent en ne prenant que ${ }^{L} G=P G L(2, \mathbb{C})$, plutôt qu'un produit $P G L(2, \mathbb{C}) \times \operatorname{Gal}(K / F),[K: F]>1$. Les groupes ${ }^{\lambda} H$ pertinents sont alors soit finis et de types très particuliers, tetraédraux, octaédraux et icosaédraux, ou, pour les groupes diédraux, des extensions du groupe $\hat{H}$ par un groupe de Galois d'ordre 2. Nous avons implicitement profité de cette classification dans la section $§ 5$. Les groupes finis abéliens ou diédraux causent des petits problèmes car leurs homomorphismes dans ${ }^{L} G$ se factorisent par un groupe diédral continu.

\section{REFERENCES}

[A] James G. Arthur, The Endoscopic Classification of Representations: Orthogonal and Symplectic Groups, AMS Colloquium Publications, to appear.

[FLN] Edward Frenkel, Robert Langlands et Ngô Bao Châu, à paraître dans Ann. Sci. Math. du Québec.

$[\mathrm{H}]$ Helmut Hasse, Bericht über neuere Untersuchungen und Probleme aus der Theorie der algebraischen Zahlkörper, Teil I: Klassenkörpertheorie, Teil II: Reziprozitätsgesetz, Physica-Verlag, Würzburg-Wien, 1965.

[JL] Hervé Jacquet et Robert Langlands, Automorphic forms on GL(2) SLM 114, Springer Verlag.

[Kn] Anthony Knapp, Representation theory of semisimple groups : An overview based on examples, Princeton Landmarks in Mathematics, Princeton University Press, 2001.

[Ko] Robert Kottwitz, Stable trace formula:cuspidal tempered terms, Duke Math. J. 51 (1984), 611-650.

[LL] Jean-Pierre Labesse et Robert Langlands, L-indistinguishability for SL(2), J. Can. de Math 31 (1979), 726-785.

[L1] Robert Langlands, Les débuts d'une formule des traces stable, Publ. Math. de l'Univ. Paris VII, 1981.

[L2] _ Beyond endoscopy, http://publications.ias.edu.

[SS] Paul Sally et Joseph Shalika, Characters of the discrete series of representations of SL(2) over a local field, PNAS 61 (1968).

[S] Joseph Shalika, A theorem on semi-simple p-adic groups, Ann. of Math. 95 (1972).

[T] J. B. Tunnell, Local $\epsilon$-Factors and Characters of GL(2), Amer. Jour. of Math. 105 (1983), The Johns Hopkins University Press, 1277-1307.

[V] V.S. Varadarajan, Harmonic analysis on real reductive groups SLN 576, Springer Verlag, 1977. 